\title{
VALVELESS PIEZOELECTRIC MICROPUMP FOR FUEL DELIVERY IN DIRECT METHANOL FUEL CELL (DMFC) DEVICES
}

by

Tao Zhang

BS, Tsinghua University, P. R. China, 1997

MS, Tsinghua University, P. R. China, 2000

Submitted to the Graduate Faculty of

the School of Engineering in partial fulfillment

of the requirements for the degree of

Doctor of Philosophy

University of Pittsburgh 


\section{UNIVERSITY OF PITTSBURGH \\ SCHOOL OF ENGINEERING}

This dissertation was presented

by

Tao Zhang

It was defended on

April 11, 2005

and approved by

Dr. David H. Archer, Adjunct Professor, Department of Mechanical Engineering, Carnegie Mellon University

Dr. Minking K. Chyu, Professor, Department of Mechanical Engineering

Dr. Peyman Givi, Professor, Department of Mechanical Engineering

Dr. Laura Schaefer, Assistant Professor, Department of Mechanical Engineering

Dissertation Director: Dr. Qing-Ming Wang, Assistant Professor, Department of Mechanical Engineering 
Copyright (c) by Tao Zhang 2005 


\title{
VALVELESS PIEZOELECTRIC MICROPUMP FOR FUEL DELIVERY IN DIRECT METHANOL FUEL CELL (DMFC) DEVICES
}

\author{
Tao Zhang, PhD
}

University of Pittsburgh, 2005

Presented in this dissertation is the study of a novel fuel delivery system combined with miniaturized direct methanol fuel cell (DMFC). The core component of this system is a valveless micropump driven by ring-type piezoelectric bending actuator. By applying an alternating electrical field across the actuator, the resultant reciprocating movement of the pump diaphragm can be converted into pumping effect. Nozzle/diffuser elements are used to direct the flow. To make the power system applicable for portable electronic devices, the micropump needs to meet some specific requirements: low power consumption but sufficient fuel flow rate. In this study, both theoretical and experimental methods have been used to investigate the effects of materials properties, actuator dimensions, driving voltage, driving frequency, nozzle/diffuser dimension, and other factors on the performance of the whole system. As a result, a viable design of micropump system for fuel delivery in DMFC devices has been achieved and some further improvements are suggested.

In the beginning of this dissertation, the history, working principle, types, and current research status of both fuel cell and micropump are reviewed. Following this comprehensive introduction, the research objective to develop a novel portable power system that combines fuel cell technology and micropump technology is presented. To help design such a system, a series of analytical models are established to estimate the deflection, volume change, flow rate, and power 
consumption of the micropump. Both finite element method (FEM) and experimental method are applied to verify the models. Based on the analytical analysis, material properties and dimensions of the micropump actuator are optimized to obtain maximum flow rate and minimum power consumption. Later a separated micropump prototype has been fabricated and tested. It is observed that the performance of a DMFC device is improved by using this micropump to supply fuel. In addition, the electrochemical impedance analysis of this fuel cell device is conducted to try to understand the reasons for the performance improvement. Finally, the accomplishments of this study are summarized and future perspective is provided. 


\section{TABLE OF CONTENTS}

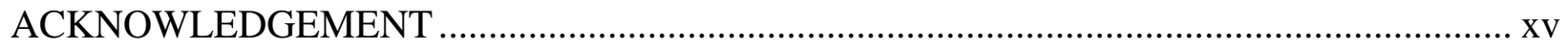

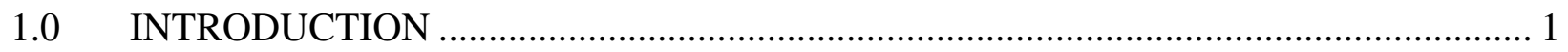

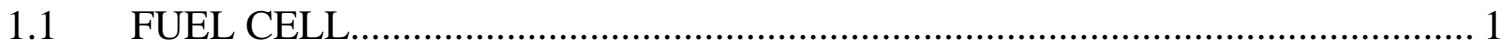

1.1.1 Fuel cell history ...................................................................... 1

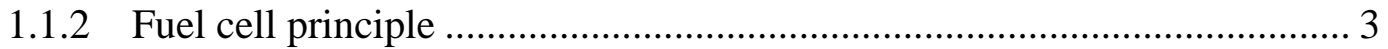

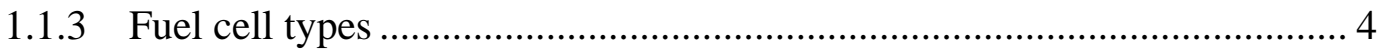

1.1.4 Current developments of fuel cell for portable electronic devices ........... 11

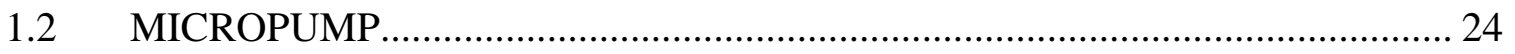

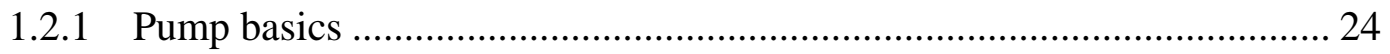

1.2.2 Micropump history................................................................... 26

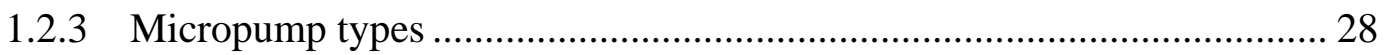

1.2.4 Current developments of valveless piezoelectric micropump .................. 36

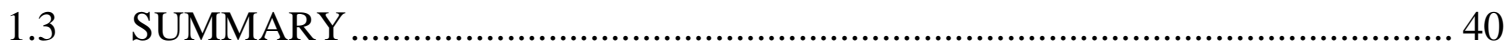

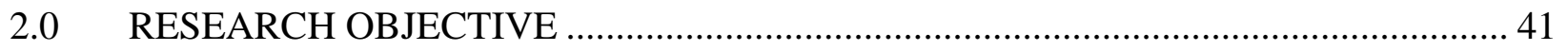

3.0 THEORETICAL ANALYSIS OF MICROPUMP ................................................ 43

3.1 DEFLECTION OF DISK-TYPE BENDING ACTUATOR ............................ 43

3.1.1 Models developed by previous researchers ..................................... 43

3.1.2 Modified model for disk-type bending actuator .................................... 54

3.2 DEFLECTION OF RING-TYPE BENDING ACTUATOR ............................. 58

3.3 VOLUME CHANGE, FLOW RATE AND POWER CONSUMPTION ............ 61 


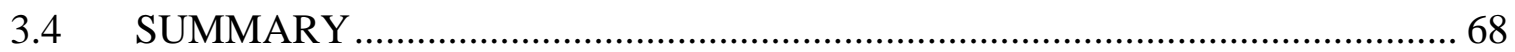

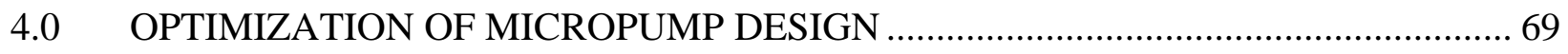

$4.1 \quad$ DISK-TYPE BENDING ACTUATOR ............................................................. 69

4.1.1 Verification by numerical simulation and experimental measurement .... 69

4.1.2 Effects of material properties.................................................................. 72

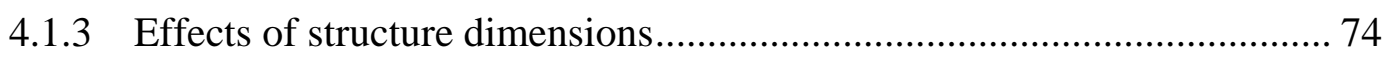

4.2 RING-TYPE BENDING ACTUATOR............................................................ 77

4.2.1 Verification by numerical simulation and experimental measurement .... 77

4.2.2 Effects of material properties............................................................... 80

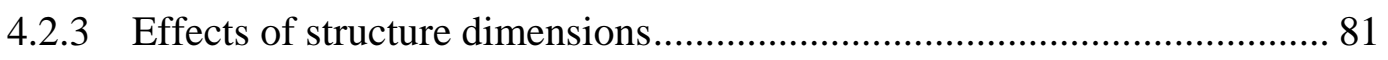

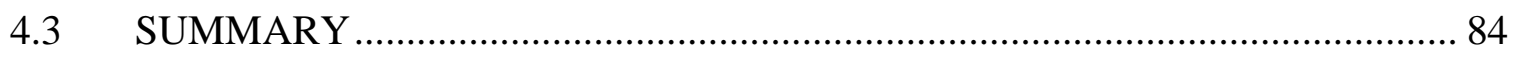

5.0 EXPERIMENTAL CHARACTERIZATION OF MICROPUMP ..................................... 86

5.1 FABRICATION OF MICROPUMP PROTOTYPE …………………………..... 86

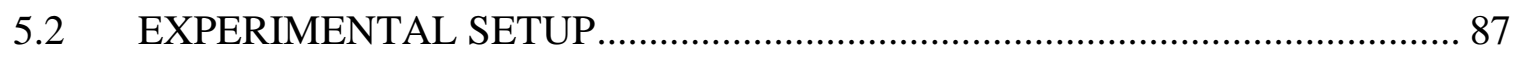

5.3 RESULTS WITHOUT LIQUID LOADING ...................................................... 88

$5.4 \quad$ RESULTS WITH LUQUID LOADING ……………....................................... 92

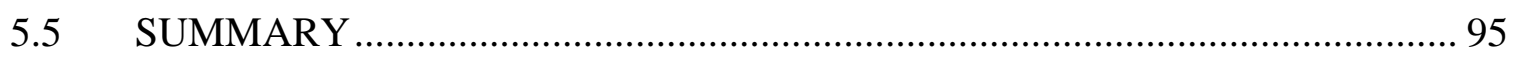

6.0 PERFORMANCE ANALYSIS OF DMFC DRIVEN BY MICROPUMP ....................... 96

6.1 ANALYTICAL ANALYSIS ..................................................................... 97

6.2 EXPERIMENTAL ANALYSIS …………………………………………….... 103

6.2.1 Performance of the DMFC device without pump..................................... 105

6.2.2 Performance of the DMFC device driven by mini pump …..................... 106

6.2.3 Performance of the DMFC device driven by micropump …………….... 108

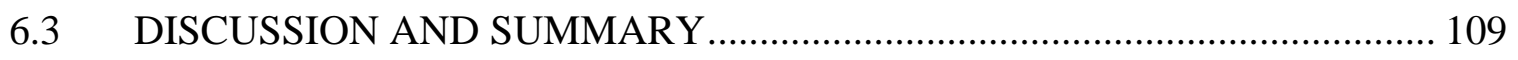

6.3.1 Open circuit voltage (OCV) of DMFC .................................................. 110 
6.3.2 Influence of pumping.......................................................................... 111

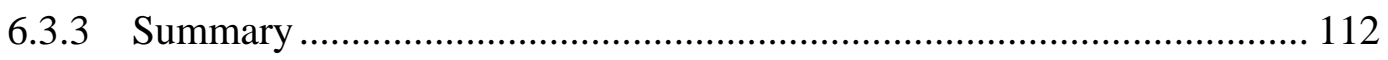

7.0 ELECTROCHEMICAL IMPEDANCE SPECTROSCOPY (EIS) ANALYSIS ............ 113

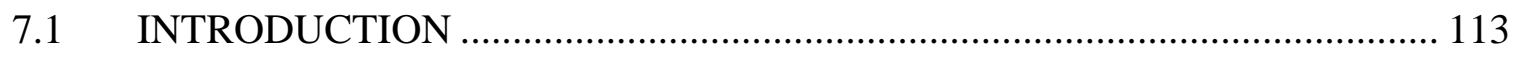

7.2 EXPERIMENTAL SETUP.................................................................. 116

7.3 RESULTS AND ANALYSIS .................................................................... 117

7.3.1 Overall EIS of DMFC ...................................................................... 117

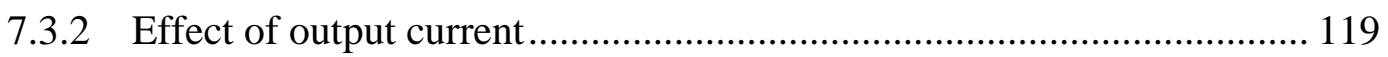

7.3.3 Effect of temperature ...................................................................... 121

7.3.4 Effect of fuel flow rate....................................................................... 121

7.3.5 Proposed equivalent circuit model......................................................... 123

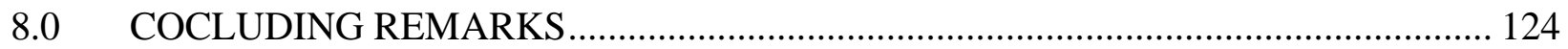

$8.1 \quad$ SUMMARY OF ACCOMPLISHMENTS ……………………………….... 124

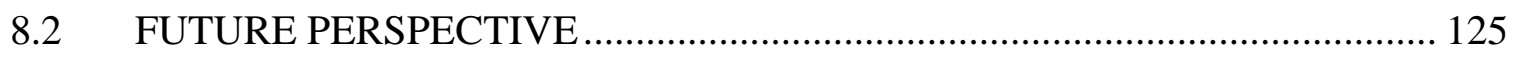

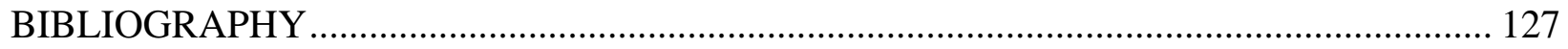




\section{LIST OF TABLES}

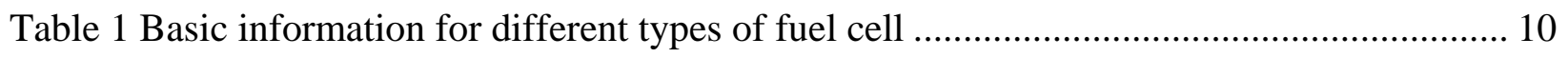

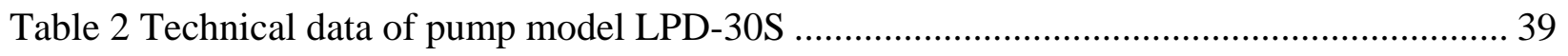

Table 3 Dimensions and material properties of the PZT disk-type bending actuator ................. 63

Table 4 Dimensions and material properties of the PZT disk-type bending actuator in [35]...... 70

Table 5 Dimensions and material properties of the PZT ring-type bending actuator.................. 77 


\section{LIST OF FIGURES}

Figure 1 William Grove's drawing of an experimental "gas battery" from an 1843 letter ............ 2

Figure 2 Schematic of an individual fuel cell ......................................................................... 3

Figure 3 Prototypes of MTI micro fuel cell system........................................................ 12

Figure 4 Intermac’s newest RFID handheld reader using Mobion ${ }^{\mathrm{TM}}$ fuel cell technology .......... 13

Figure 5 Two concept models incorporated with Mobion ${ }^{\mathrm{TM}}$ fuel cell technology ..................... 13

Figure 6 The Motorola DMFC prototype powering a PDA .............................................. 14

Figure 7 Prototype methanol micro fuel cell from Menlo Park............................................. 15

Figure 8 New electrolyte structure developed by Neah Power System................................... 15

Figure 9 Medis Technologies LTD’s power pack ........................................................... 15

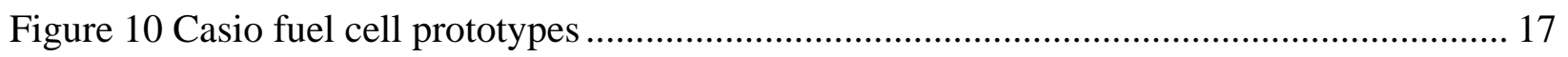

Figure 11 Hitachi fuel cell prototypes ............................................................................ 18

Figure 12 NEC fuel cell notebook prototypes from 2003 (left) to 2004 (center, right) .............. 18

Figure 13 NTT DoCoMo 3G cell phone handset powered by micro fuel cell ........................... 19

Figure 14 Toshiba prototypes fuel cell-powered PDA ...................................................... 20

Figure 15 Toshiba prototypes fuel cell-powered laptop ................................................... 20

Figure 16 Fraunhofer ISE's micro-fuel cell powering a laptop ........................................... 21

Figure 17 Illustration of waterwheels designs ................................................................. 25

Figure 18 Illustration of Archimedes’ screw pump ............................................................. 25

Figure 19 The piezoelectric micropump developed by J. G. Smits ....................................... 27

Figure 20 Reciprocating displacement micropump with single pump chamber [22] ................. 30 
Figure 21 Reciprocating displacement micropumps with different actuation mechanisms ......... 32

Figure 22 Piezoelectric valve-less micropump with conical nozzle/diffuser elements ............... 33

Figure 23 Piezoelectric valve-less micropump with pyramidic nozzle/diffuser elements ........... 34

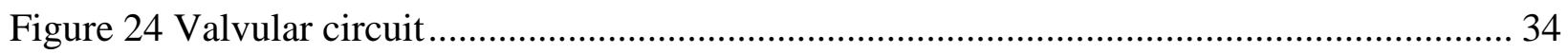

Figure 25 Photo of the micropump prototypes developed by PAR Technologies, Inc. ............... 39

Figure 26 Schematic of the miniaturized direct methanol fuel cell (DMFC) power system using valveless piezoelectric micropump for fuel delivery............................................ 41

Figure 27 Schematic of the pump actuator model in [47] ................................................. 44

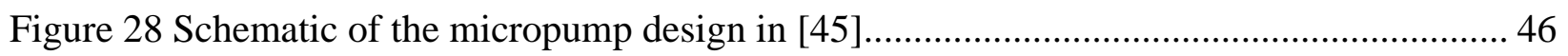

Figure 29 Schematic of the PZT disk-type bending actuator .............................................. 46

Figure 30 Schematic of the pump actuator model for analysis............................................. 46

Figure 31 Strain and stress distribution of the three-layer structure ..................................... 47

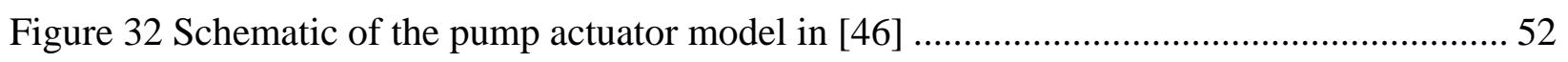

Figure 33 Schematic of the PZT disk-type bending actuator model ...................................... 55

Figure 34 Schematic of the PZT ring-type bending actuator model....................................... 58

Figure 35 Comparison of the diaphragm deflections under 100 volts and $1 \mathrm{kPa}$ separately........ 62

Figure 36 Deformation results of the PZT disk-type bending actuator using ANSYS .............. 71

Figure 37 Comparison of the diaphragm deflections for the PZT disk-type bending actuator

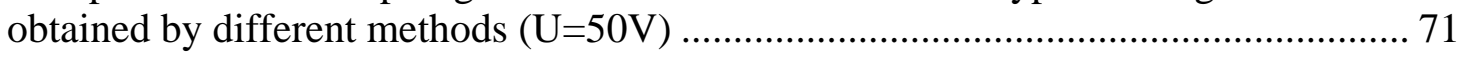

Figure 38 Relationship between the proportional coefficient $k_{U}$ and the PZT layer Young's

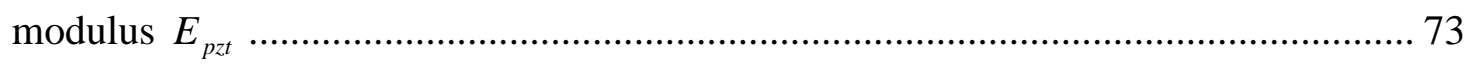

Figure 39 Relationship between the proportional coefficient $k_{U}$ and the passive plate Young's

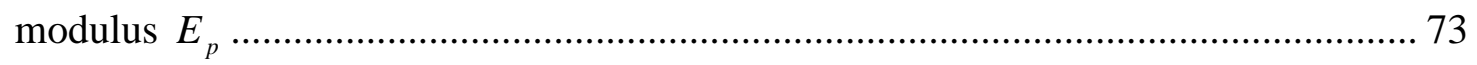

Figure 40 Coefficient $k_{U}$ and $k_{U}^{2} / C_{0}$ at different PZT/passive plate radius ratio..................... 75

Figure 41 Coefficient $k_{U}$ and $k_{U}^{2} / C_{0}$ at different passive plate radius with fixed PZT/passive

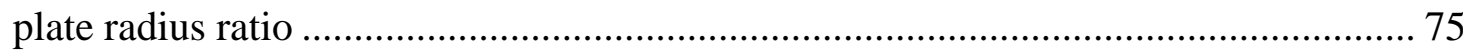


Figure 42 Coefficient $k_{U}$ and $k_{U}^{2} / C_{0}$ at different PZT layer thickness.

Figure 43 Coefficient $k_{U}$ and $k_{U}^{2} / C_{0}$ at different passive/PZT layer thickness ratio with fixed

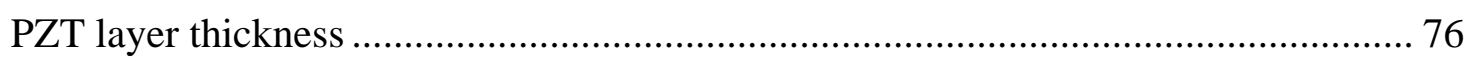

Figure 44 Deformation results of the PZT ring-type bending actuator using ANSYS............... 79

Figure 45 Comparison of the diaphragm deflections for the PZT ring-type bending actuator obtained by different methods $(\mathrm{U}=100 \mathrm{~V})$........................................................ 79

Figure 46 Relationship between the proportional coefficient $k_{U}$ and the PZT layer Young's

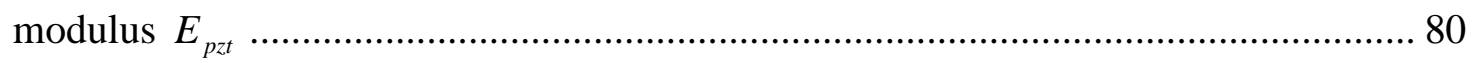

Figure 47 Relationship between the proportional coefficient $k_{U}$ and the passive plate Young's

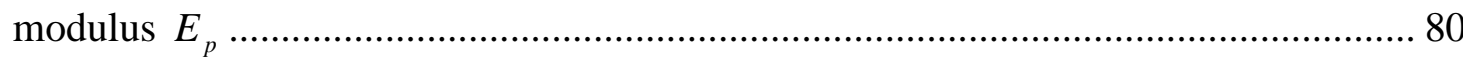

Figure 48 Coefficient $k_{U}$ and $k_{U}^{2} / C_{0}$ at different PZT/passive plate radius ratio.................... 81

Figure 49 Coefficient $k_{U}$ and $k_{U}^{2} / C_{0}$ at different passive plate radius with fixed PZT/passive

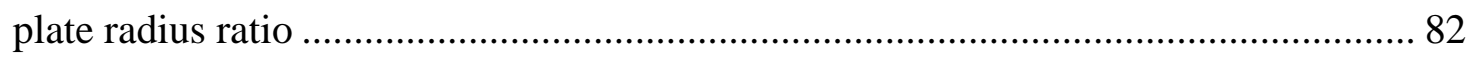

Figure 50 Coefficient $k_{U}$ and $k_{U}^{2} / C_{0}$ at different PZT layer thickness................................. 83

Figure 51 Coefficient $k_{U}$ and $k_{U}^{2} / C_{0}$ at different passive/PZT layer thickness ratio with fixed

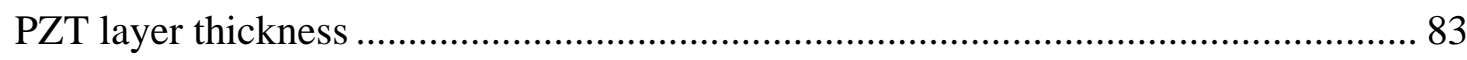

Figure 52 Design graph of the micropump housing ........................................................ 86

Figure 53 Photo of the piezoelectric valveless micropump prototype.................................... 86

Figure 54 Experimental setup for the micropump measurement ........................................... 88

Figure 55 Measured center deflection of the micropump diaphragm under different applied DC

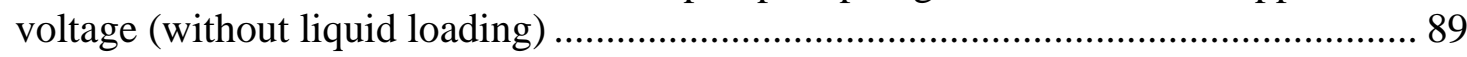

Figure 56 Measured center deflection of the micropump diaphragm under different applied AC

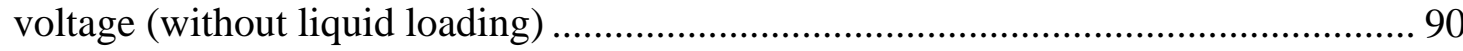

Figure 57 Measured deflection distribution of the micropump diaphragm under applied DC and

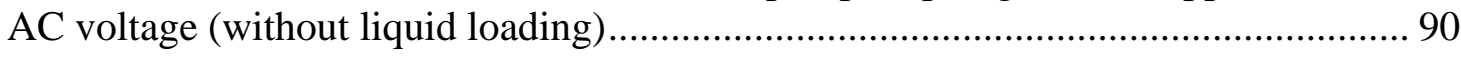

Figure 58 Frequency response of the micropump obtained by different methods (without liquid loading)..... 91 
Figure 59 Measured center deflection of the micropump diaphragm under different applied AC voltage (with liquid loading)

Figure 60 Frequency response of the micropump obtained by different methods (with liquid

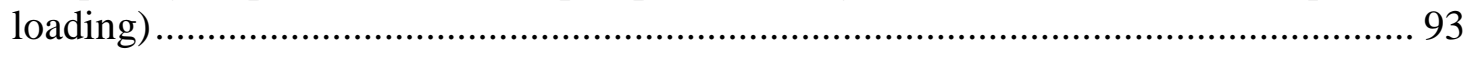

Figure 61 Measured flow rate versus pressure head (water as working fluid) .............................. 94

Figure 62 Measured pressure head at zero flow rate versus driving frequency (Vrms=100V).... 94

Figure 63 Selected experimental J-V data of a single fuel cell at different fuel flow rate [61].... 99

Figure 64 Calculated power density of a single fuel cell at different fuel flow rate..................... 99

Figure 65 Net power output under different operation conditions (Actuation frequency $=100 \mathrm{~Hz}$ )

Figure 66 Net power output under different operation conditions (Actuation frequency $=200 \mathrm{~Hz}$ )

Figure 67 Photo of the DMFC device used in the experiments.................................................... 104

Figure 68 Photo of the peristaltic variable flow mini pump ……….............................................. 104

Figure 69 Measured characteristic curves of the DMFC device using different concentration of

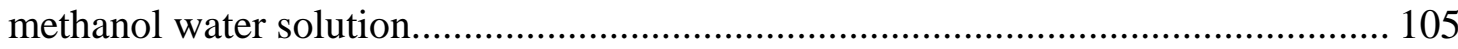

Figure 70 Calculated power of the DMFC device using different concentration of methanol water

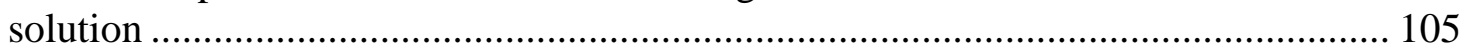

Figure 71 Measured characteristic curves of the DMFC device driven by mini pump ............... 107

Figure 72 Calculated power of the DMFC device driven by mini pump ................................... 107

Figure 73 Measured characteristic curves of the DMFC device driven by micropump .............. 108

Figure 74 Calculated power of the DMFC device driven by micropump ................................... 108

Figure 75 A simple electrochemical reaction and its equivalent circuit.................................... 114

Figure 76 Experimental setup for the impedance measurement of a fuel cell device ................. 116

Figure 77 Measured impedance amplitude of a fuel cell device versus frequency ..................... 117

Figure 78 Measured impedance phase shift of a fuel cell device versus frequency .................... 118

Figure 79 Nyquist plot of a fuel cell device............................................................................. 118 
Figure 80 Measured Nyquist plot of a fuel cell device with open circuit (room temperature, no

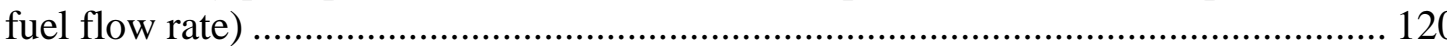

Figure 81 Measured Nyquist plot of a fuel cell device under different output current (room temperature, no fuel flow rate)

Figure 82 Measured Nyquist plot of a fuel cell device under different temperature (output current is $0.1 \mathrm{~A}$, fuel flow rate is $5 \mathrm{ml} / \mathrm{min}$ ). 121

Figure 83 Measured Nyquist plot of a fuel cell device under different fuel flow rate (room temperature, output current is $0.1 \mathrm{~A})$......................................................................... 122

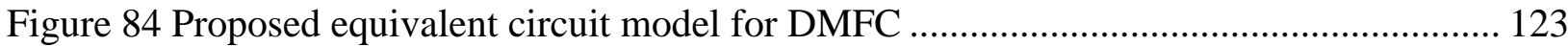




\section{ACKNOWLEDGEMENT}

The work in this dissertation has been carried out at the Microsensor and Microactuator Laboratory, Department of Mechanical Engineering, University of Pittsburgh, Pittsburgh, USA. Financial support has been received from the Pittsburgh Digital Greenhouse, a State High-Tech Consortium with research and development focus on System-On-A-Chip technology advancement. It is also partially supported by Department of Energy (DOE) and University of Pittsburgh Small Grant Funding.

Many people have supported and helped me in this work and I am very grateful to them.

First I would like to thank Dr. Qing-Ming Wang, my advisor for the tremendous guidance and support he has been given me since I started my PhD study at University of Pittsburgh. He is an excellent scientist, engineer, teacher and truly a friend. I have benefited a lot from him both professionally and personally. It is really a great experience to study with him.

Special thanks to Dr. David H. Archer for leading me into the world of fuel cell. I am really grateful to his wonderful teaching and helpful discussion, which aided me greatly in this research.

Many thanks to Dr. Minking K. Chyu, who initiated the fuel cell research in our department. I appreciate the supports and advices he has provided me with.

I am also grateful to the other committee members: Dr. Peyman Givi and Dr. Laura Schaefer for their helpful insights and contributions. 
Dr. Peiwen Li has been working with me in the research of polymer electrolyte fuel cell. I appreciate his help and assistance deeply.

I want to thank all the different friends that have been a part of my life during the last five years and I really enjoyed their friendship.

Finally, a very special thank you to my parents: Guangfan Zhang and Yunxian Yu, my brother: Wei Zhang, and my best friend: Miao Yu for their love, support, and faith in me. Thanks! 


\subsection{INTRODUCTION}

Energy was, is and will always be playing a vital role in human history. The never-ending pursuit of efficient and sustainable energy system consists one of the most important parts of modern science. The objective of this research is to explore the possibility of applying miniaturized fuel cell power system to portable electronic devices and most of the discussions are focused on the fuel delivery problem.

\subsection{FUEL CELL}

\subsubsection{Fuel cell history}

Unlike other new technologies, fuel cell actually has a relatively long history. William Robert Grove, an English lawyer turned scientist, developed the first fuel cell in 1839. As show in Fig. 1 from his 1843 letter “On the Gas Voltaic Battery”, he put oxygen and hydrogen into a series of separated tubes then filled between them with dilute sulphuric acid (left side of Fig. 1). The platinum electrodes were immersed into each tube then connected to an interposed voltmeter (right side of Fig. 1). He observed that a constant current would flow between the electrodes and the water level rose at the same time. 


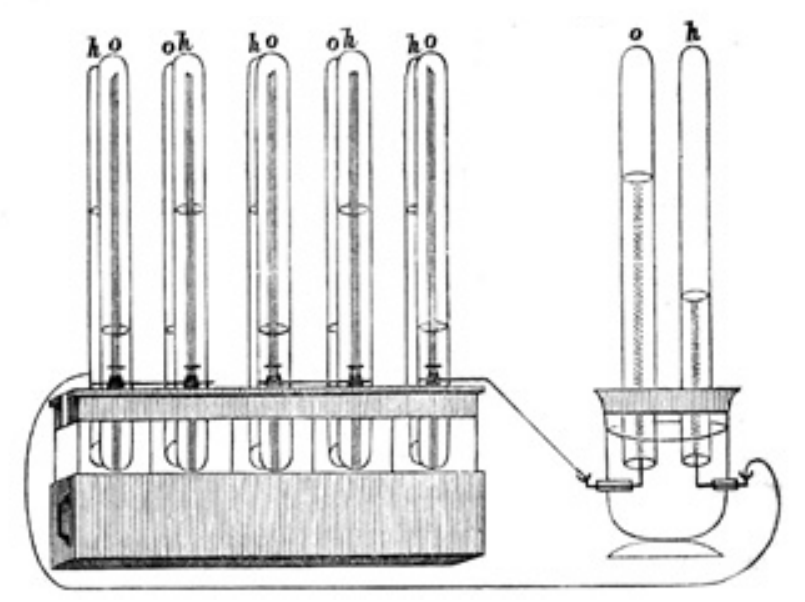

Figure 1 William Grove's drawing of an experimental "gas battery" from an 1843 letter What happened here was actually a reverse reaction of the electrolysis of water. This reaction represented by Eq. (1.1) is the same as that of burning hydrogen directly. The difference is that electricity energy is generated in fuel cell while only heat is generated in direct burning. This device also showed clearly that both oxygen and hydrogen were required to produce current.

$$
\mathrm{H}_{2}+\frac{1}{2} \mathrm{O}_{2} \rightarrow \mathrm{H}_{2} \mathrm{O}
$$

After Grove's invention, many researchers have been seeking various ways to improve the performance of fuel cell. In 1889, chemist Ludwig Mond and his assistant Carl Langer made a hydrogen/oxygen fuel cell that attained 6 amps current per square foot at 0.73 volts. The electrode they used was thin, perforated platinum and the electrolyte was in a quasi-solid form. In 1896, an electric engineer and chemist, William W. Jacques tried to make electricity directly from coal based on fuel cell principle. He used alkali as electrolyte and carbon as electrode, but failed to get an electrochemical reaction from his "carbon battery". Emil Baur from Switzerland conducted research on different fuel cells, including high temperature device (using molten silver as electrolyte) and a unit that used clay and metal oxides as a sold electrolyte. Friedrich Wilhelm Ostwald revealed the underlying chemistry theory of fuel cell operation in 1893. 
Although a lot of theoretical and experimental studies have been focused on fuel cell since 1839, the first successful fuel cell device has not been developed until 1930s. Francis Thomas Bacon, an English engineer, designed a hydrogen-oxygen cell using less corrosive alkaline electrolytes and inexpensive nickel electrodes in 1932. Later he and his coworkers demonstrated a practical five-kilowatt fuel cell system in 1959. Based on the Bacon’s patents, NASA has built compact alkaline fuel cells to provide electricity and clean water on spacecrafts since early 1960s. These fuel cells operated successfully in the manned moon flights and subsequent space missions.

As a clean and efficient power generation device, fuel cell has shown a great potential in a lot of fields such as stationary power, residential power, transportation, portable power and landfill/wastewater treatment. So far, many have already been in use and many are still in development.

\subsubsection{Fuel cell principle}

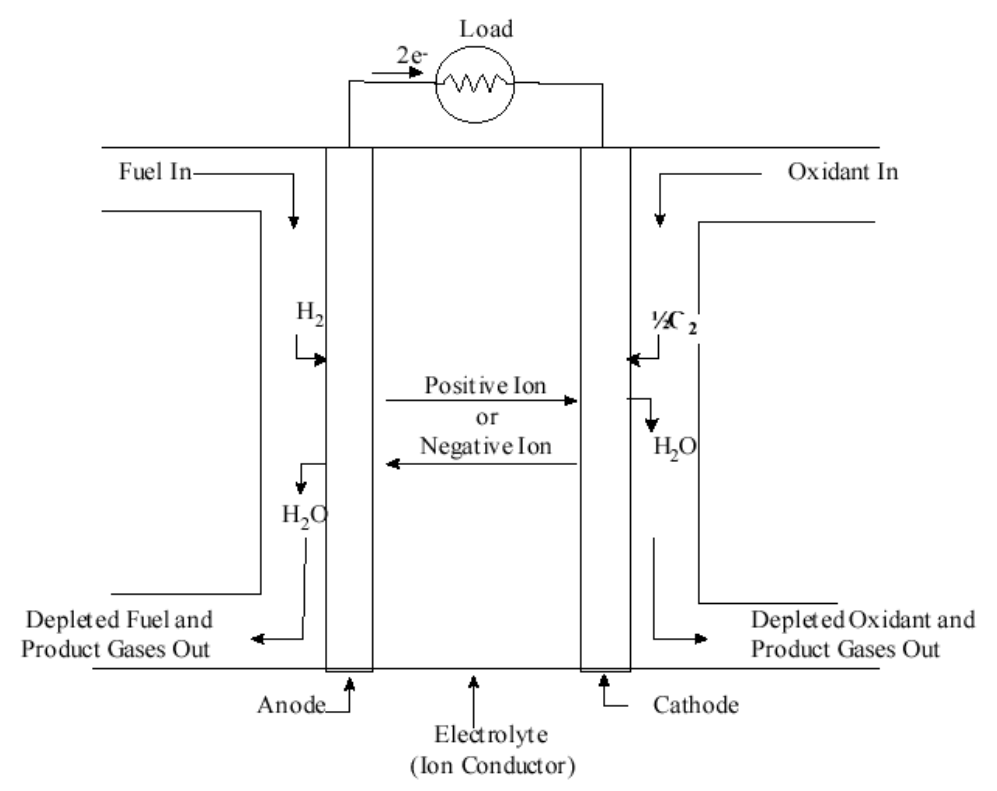

Figure 2 Schematic of an individual fuel cell 
As shown in Fig. 2, a basic fuel cell consists of three components: anode, electrolyte and cathode. When fuel cell is working, fuel is continuously fed into anode while oxidant gas is continuously fed into cathode. Electrochemical reactions occur at the interface between electrodes and electrolyte. The ions flowing inside electrolyte and the electrons passing through the outer load form a close circuit. Other reaction products include heat, water and carbon dioxide (if use hydrocarbon as fuel directly).

\subsubsection{Fuel cell types}

Based on the type of electrolyte used, fuel cells can be classified as polymer electrolyte fuel cell (PEFC), alkaline fuel cell (AFC), phosphoric acid fuel cell (PAFC), molten carbonate fuel cell (MCFC), solid oxide fuel cell (SOFC), and protonic ceramic fuel cell (PCFC). There are also some other types of fuel cells, such as direct methanol fuel cell (DMFC), zinc air fuel cell (ZAFC), bio-fuel cell (BFC), and regenerative fuel cell (RFC).

\section{Polymer electrolyte fuel cell (PEFC)}

This fuel cell utilizes an ion exchange membrane (fluorinated sulfonic acid polymer or other similar polymer) as electrolyte. Hydrogen ions can pass through this membrane while gas molecules and electrons cannot. The electrochemical reactions of this fuel cell are:

Anode: $\mathrm{H}_{2} \rightarrow 2 \mathrm{H}^{+}+2 e^{-}$

$$
\text { Cathode: } \frac{1}{2} \mathrm{O}_{2}+2 \mathrm{H}^{+}+2 e^{-} \rightarrow \mathrm{H}_{2} \mathrm{O}
$$

$$
\text { Overall: } \mathrm{H}_{2}+\frac{1}{2} \mathrm{O}_{2} \rightarrow \mathrm{H}_{2} \mathrm{O}
$$

Therefore, fuel is always hydrogen-rich gas and oxidant is oxygen or air. 
Due to the limitation imposed by polymer membrane, the operation temperature is quite low (below $80^{\circ} \mathrm{C}$ ). To speed the reactions at low temperature, a catalyst layer ( $\mathrm{Pt}$ or $\mathrm{Pt} / \mathrm{Ru}$ in most cases) is required for both anode and cathode. On the other hand, the low working temperature makes this fuel cell an ideal candidate for buildings, vehicles and portable electronics power applications. The main technique issues associated with this fuel cell are water balance, catalyst loading, manufacture cost and fuel storage.

\section{Direct methanol fuel cell (DMFC)}

DMFC uses the similar electrolyte as PEFC but employ methanol water solution as fuel. Since the methanol permeation rate is relatively high for ordinary PEFC membrane, some modifications have to be made, such as increasing the thickness of the membrane, adding barrier layer, changing the composition and the morphology of the membrane. The electrochemical reactions of DMFC are:

$$
\begin{gathered}
\text { Anode: } \mathrm{CH}_{3} \mathrm{OH}+\mathrm{H}_{2} \mathrm{O} \rightarrow \mathrm{CO}_{2}+6 \mathrm{H}^{+}+6 e^{-} \\
\text {Cathode: } \frac{3}{2} \mathrm{O}_{2}+6 \mathrm{H}^{+}+6 e^{-} \rightarrow 3 \mathrm{H}_{2} \mathrm{O} \\
\text { Overall: } \mathrm{CH}_{3} \mathrm{OH}+\frac{3}{2} \mathrm{O}_{2} \rightarrow \mathrm{CO}_{2}+2 \mathrm{H}_{2} \mathrm{O}
\end{gathered}
$$

DMFC also has the same working temperature and applications as PEFC. In comparisons with hydrogen, methanol solution is easier to store and the energy density is higher, therefore DMFC is more suitable for portable power applications. However, the reaction rate of DMFC is more sluggish and the fuel crossing over problem can bring extra loss to the performance.

\section{Alkaline fuel cell (AFC)}

The electrolyte used in this fuel cell is an aqueous solution of alkaline potassium hydroxide $(\mathrm{KOH})$ soaked in a matrix (usually asbestos). For different operation temperature, the 
concentration of $\mathrm{KOH}$ may vary. Both fuel (hydrogen) and oxidant (oxygen or air) have to be in high purity without reactive constituents in case the electrolyte will be altered. The electrochemical reactions of AFC are:

$$
\begin{gathered}
\text { Anode: } \mathrm{H}_{2}+2 \mathrm{OH}^{-} \rightarrow 2 \mathrm{H}_{2} \mathrm{O}+2 e^{-} \\
\text {Cathode: } \frac{1}{2} \mathrm{O}_{2}+\mathrm{H}_{2} \mathrm{O}+2 e^{-} \rightarrow 2 \mathrm{OH}^{-} \\
\text {Overall: } \mathrm{H}_{2}+\frac{1}{2} \mathrm{O}_{2} \rightarrow \mathrm{H}_{2} \mathrm{O}
\end{gathered}
$$

The operation temperature of $\mathrm{AFC}$ is from $65^{\circ} \mathrm{C}$ to $220^{\circ} \mathrm{C}$. NASA has used it on space missions since 1960s, which can provide both electricity and drinking water for spacecraft. However, this fuel cell is too expensive for commercial applications.

\section{Phosphoric acid fuel cell (PAFC)}

The electrolyte used in this fuel cell is $100 \%$ phosphoric acid. The matrix used to contain the electrolyte is often silicon carbide and Pt as catalyst is required at both electrodes. PAFC can tolerate a small amount of carbon dioxide so it can use hydrogen rich gas with $\mathrm{CO}_{2}$ existence. The electrochemical reactions of PAFC are:

Anode: $\mathrm{H}_{2} \rightarrow 2 \mathrm{H}^{+}+2 e^{-}$

$$
\begin{gathered}
\text { Cathode: } \frac{1}{2} \mathrm{O}_{2}+2 \mathrm{H}^{+}+2 e^{-} \rightarrow \mathrm{H}_{2} \mathrm{O} \\
\text { Overall: } \mathrm{H}_{2}+\frac{1}{2} \mathrm{O}_{2} \rightarrow \mathrm{H}_{2} \mathrm{O}
\end{gathered}
$$

The operation temperature of PAFC is from $150^{\circ} \mathrm{C}$ to $220^{\circ} \mathrm{C}$. It is reported that there are more than 200 such fuel cell systems installed all over the world - mostly used in buildings. However, the requirement of high cost precious metal as catalyst and CO intolerance limit its further development. 


\section{Molten carbonate fuel cell (MCFC)}

MCFC uses a combination of alkali carbonates retained in a ceramic matrix of $\mathrm{LiAlO}_{2}$ as electrolyte. At high temperature $\left(600^{\circ} \mathrm{C}-700^{\circ} \mathrm{C}\right)$, alkali carbonates form highly conducive molten salts that can let carbonate ions pass through. Noble metal catalysts are not required and the fuel selection range is very wide: hydrogen, carbon monoxide, natural gas, propane, diesel and etc. The primary electrochemical reactions of MCFC are:

$$
\begin{aligned}
& \text { Anode: } \mathrm{H}_{2}+\mathrm{CO}_{3}^{2-} \rightarrow \mathrm{H}_{2} \mathrm{O}+\mathrm{CO}_{2}+2 e^{-} \\
& \text {Cathode: } \frac{1}{2} \mathrm{O}_{2}+\mathrm{CO}_{2}+2 e^{-} \rightarrow \mathrm{CO}_{3}^{2-} \\
& \text { Overall: } \mathrm{H}_{2}+\frac{1}{2} \mathrm{O}_{2}+\mathrm{CO}_{2} \rightarrow \mathrm{H}_{2} \mathrm{O}+\mathrm{CO}_{2}
\end{aligned}
$$

MCFC has mainly been used in electric utility applications and stationary applications. The disadvantages of MCFC includes highly corrosive and mobile electrolyte, $\mathrm{CO}_{2}$ source requirement at cathode to form carbonate ions, material problem due to high working temperature and etc.

\section{Solid oxide fuel cell (SOFC)}

The electrolyte in SOFC is ceramic which mainly consists of a solid, nonporous metal oxide, usually Y2O3-stabilized ZrO2. Mostly, the anode is Ni-YSZ cermet and the cathode is Sr-doped LaMnO3. SOFC has two work temperature ranges: $600^{\circ} \mathrm{C}-800^{\circ} \mathrm{C}$ and $800^{\circ} \mathrm{C}-1000^{\circ} \mathrm{C}$. The cell shape of SOFC can be either planar or tubular. At high temperature, the electrolyte is conductive for oxygen ions. Also to improve the ionic conduction, thin film technology has been widely introduced in the production of SOFC cell. Alternative electrolyte and electrodes are also being developed. The primary electrochemical reactions of SOFC are:

$$
\text { Anode: } \mathrm{H}_{2}+\mathrm{O}^{2-} \rightarrow \mathrm{H}_{2} \mathrm{O}+2 e^{-}
$$




$$
\begin{aligned}
& \text { Cathode: } \frac{1}{2} \mathrm{O}_{2}+2 e^{-} \rightarrow \mathrm{O}^{2-} \\
& \text { Overall: } \mathrm{H}_{2}+\frac{1}{2} \mathrm{O}_{2} \rightarrow \mathrm{H}_{2} \mathrm{O}
\end{aligned}
$$

The commercialization of SOFC is mainly focused on industry and large-scale electrical power stations. However, some companies are also trying to develop SOFC for motor vehicles and other small-scale applications. The high working temperature brings about a lot of technique challenges in the fabrication of SOFC, especially the sealing between cells. How to decrease the manufacturing cost is the key to the successful commercial applications of SOFC.

\section{Zinc air fuel cell (ZAFC)}

A typical ZAFC consists of a gas diffusion electrode (GDE), a zinc anode separated by electrolyte (usually concentrated $\mathrm{KOH}$ ), and some mechanical separators. Oxygen can pass through the GDE and form hydroxyl ions. The hydroxyl ions then travel through the electrolyte to the Zinc anode and reacts with Zinc. The electrochemical reactions of ZAFC are:

$$
\begin{gathered}
\text { Anode: } \mathrm{Zn}+2 \mathrm{OH}^{-} \rightarrow \mathrm{ZnO}+\mathrm{H}_{2} \mathrm{O}+2 e^{-} \\
\text {Cathode: } \frac{1}{2} \mathrm{O}_{2}+\mathrm{H}_{2} \mathrm{O}+2 e^{-} \rightarrow 2 \mathrm{OH}^{-} \\
\text {Overall: } \mathrm{Zn}+\frac{1}{2} \mathrm{O}_{2} \rightarrow \mathrm{ZnO}
\end{gathered}
$$

After the Zinc is used up, a recycling/refueling system uses electrolysis to convert the zinc oxide back to zinc metal in pellet form using AC electricity. Based on the similar principle, other metal can also be used as fuel for the metal-air fuel cell, such as aluminum air fuel cell. This type of fuel cell has a potential wide range of applications, ranging from electrical vehicles, commercial electronics to military. The extra recycling/refueling system and the efficiency are the main drawbacks of this fuel cell. 


\section{Protonic ceramic fuel cell (PCFC)}

This fuel cell is based on a ceramic electrolyte material that has high protonic conductivity at high temperatures. At $700^{\circ} \mathrm{C}$, together with the same thermal and kinetic advantages as MCFC and SOFC, PCFC exhibit all the intrinsic benefits of proton conduction in PEFC and PAFC. Therefore, the primary electrochemical reactions of PCFC are as same as PEFC and PAFC.

High operating temperature is necessary to achieve high fuel efficiency with hydrocarbon fuels. PCFC can operate at high temperatures and utilize fossil fuels directly, which eliminates the costly reforming process. Gaseous molecules of the hydrocarbon fuel are absorbed on the surface of the anode in the presence of water vapor, and separated hydrogen ions pass through the electrolyte, with carbon dioxide as the primary reaction product. Additionally, PCFC have a solid electrolyte so water management becomes much easier. Protonetics International Inc. first developed this type of fuel cell.

\section{Regenerative fuel cell (RFC)}

Regenerative fuel cell provides a closed-loop form of power generation. Water is first separated into hydrogen and oxygen using a solar-powered electrolyser. Then hydrogen and oxygen are fed into the fuel cell to generate electricity, heat and water. The water is then recirculated back to the solar-powered electrolyser and the process repeats again. The electrochemical reactions of RFC are as same as PEFC. This is an attractive method to store and covert solar energy. NASA and others developers worldwide are currently investigating this type of fuel cell.

\section{Bio-fuel cell (BFC)}

Bio-fuel cell ranges from microbially sustained cells at the seawater/sediment interface or in waste streams, to highly engineered bioanodes and biocathodes using redox enzymes. The electrochemical reactions of microbial BFC using acetic acid as fuel are: 
Anode: $\mathrm{C}_{2} \mathrm{H}_{4} \mathrm{O}_{2}+2 \mathrm{H}_{2} \mathrm{O} \rightarrow 8 \mathrm{H}^{+}+2 \mathrm{CO}_{2}+8 e^{-}$

Cathode: $2 \mathrm{O}_{2}+8 \mathrm{H}^{+}+8 e^{-} \rightarrow 4 \mathrm{H}_{2} \mathrm{O}$

Overall: $\mathrm{C}_{2} \mathrm{H}_{4} \mathrm{O}_{2}+2 \mathrm{O}_{2} \rightarrow 2 \mathrm{H}_{2} \mathrm{O}+2 \mathrm{CO}_{2}$

The research issues with microbial fuel cell include choice of external or internal electron mediators to achieve useful current densities, elucidation, and exploitation of the complex set of redox reactions and gradients at the electrodes, and microbial selection and immobilization. Biofuel cell utilizing engineered redox enzymes at both electrodes requires all catalytic components to be immobilized (i.e., non-freely diffusible) at high density and correctly oriented. Mechanisms for rapid electron and hydride transfer between the electrodes and the biocatalytic redox center need to be developed. Also of interest are experimental and theoretical approaches to bio-mimic catalysts able to activate C-H bonds at ambient temperatures in fuels such as alkanes.

Basic information about these various types of fuel cell is summarized in Table 1.

Table 1 Basic information for different types of fuel cell

\begin{tabular}{|c|c|c|c|c|}
\hline Fuel Cell Type & Fuel & Electrolyte & $\begin{array}{l}\text { Operation } \\
\text { Temperature }\end{array}$ & Applications \\
\hline PEFC & $\begin{array}{l}\text { Hydrogen } \\
\text { Gas }\end{array}$ & $\begin{array}{c}\text { Ion Exchange } \\
\text { Membrane }\end{array}$ & $<80^{\circ} \mathrm{C}$ & $\begin{array}{l}\text { Mobile and portable } \\
\text { applications, also for lower } \\
\text { power CHP systems. }\end{array}$ \\
\hline DMFC & $\begin{array}{l}\text { Methanol } \\
\text { Water } \\
\text { Solution }\end{array}$ & $\begin{array}{l}\text { Ion Exchange } \\
\text { Membrane }\end{array}$ & $<80^{\circ} \mathrm{C}$ & $\begin{array}{l}\text { Portable power } \\
\text { applications. }\end{array}$ \\
\hline AFC & $\begin{array}{l}\text { Hydrogen } \\
\text { Gas }\end{array}$ & $\begin{array}{c}\text { Mobilized or } \\
\text { immobilized } \\
\text { Potassium } \\
\text { Hydroxide }\end{array}$ & $65^{\circ} \mathrm{C}-220^{\circ} \mathrm{C}$ & NASA space missions. \\
\hline PAFC & $\begin{array}{l}\text { Hydrogen } \\
\text { Gas }\end{array}$ & $\begin{array}{l}\text { Immobilized } \\
\text { Liquid } \\
\text { Phosphoric } \\
\text { Acid } \\
\end{array}$ & $150^{\circ} \mathrm{C}-220^{\circ} \mathrm{C}$ & $\begin{array}{l}\text { Many } 200 \mathrm{~kW} \text { CHP } \\
\text { systems in use. }\end{array}$ \\
\hline MCFC & $\begin{array}{l}\text { Gaseous } \\
\text { Hydrocarbon } \\
\text { Fuel }\end{array}$ & $\begin{array}{c}\text { Immobilized } \\
\text { Liquid } \\
\text { Molten } \\
\text { Carbonate }\end{array}$ & $600^{\circ} \mathrm{C}-700^{\circ} \mathrm{C}$ & $\begin{array}{l}\text { Medium to large scale } \\
\text { CHP systems, up to MW } \\
\text { capacity }\end{array}$ \\
\hline
\end{tabular}




\begin{tabular}{|c|c|c|c|c|}
\hline SOFC & $\begin{array}{c}\text { Gaseous } \\
\text { Hydrocarbon } \\
\text { Fuel }\end{array}$ & Ceramic & $\begin{array}{c}600^{\circ} \mathrm{C}-800^{\circ} \mathrm{C} \\
\text { or } \\
800^{\circ} \mathrm{C}-1000^{\circ} \mathrm{C}\end{array}$ & $\begin{array}{l}\text { All sizes of CHP systems, } \\
2 \mathrm{~kW} \text { to multi MW. }\end{array}$ \\
\hline ZAFC & Zinc & $\begin{array}{l}\text { Concentrated } \\
\text { Potassium } \\
\text { Hydroxide }\end{array}$ & $<70^{\circ} \mathrm{C}$ & $\begin{array}{l}\text { Mobile and portable } \\
\text { applications. }\end{array}$ \\
\hline PCFC & $\begin{array}{c}\text { Gaseous } \\
\text { Hydrocarbon } \\
\text { Fuel }\end{array}$ & Ceramic & $\sim 700^{\circ} \mathrm{C}$ & CHP systems. \\
\hline RFC & $\begin{array}{l}\text { Hydrogen } \\
\text { Gas }\end{array}$ & $\begin{array}{l}\text { Ion Exchange } \\
\text { Membrane }\end{array}$ & $<80^{\circ} \mathrm{C}$ & NASA space missions. \\
\hline $\mathrm{BFC}$ & Biomass & $\begin{array}{l}\text { Seawater or } \\
\text { Waste } \\
\text { Streams }\end{array}$ & $\begin{array}{c}\text { Ambient } \\
\text { Temperature }\end{array}$ & $\begin{array}{l}\text { Small power sources for } \\
\text { micro sensors, micro } \\
\text { actuators and telemetry } \\
\text { devices }\end{array}$ \\
\hline
\end{tabular}

\subsubsection{Current developments of fuel cell for portable electronic devices}

As recently reported [1], there exists a great need for a long lifetime, high-efficiency power generation system with compact size to provide the driving power for portable or self-sustaining electronic systems. Of all kinds of fuel cells, PEFC and DMFC can deliver high power density and operate at low temperature, allowing for faster startups and immediate response to changes in the demand for power. Together with low weight, cost, and compact volume, PEFC and DMFC are believed to be the ideal power generation source for powering electronic devices. However, as the development of other miniaturized devices, many issues related to the design, fabrication and performance evaluation of PEFC and DMFC in the small scales still remain to be solved for the actual applications of power devices.

Up to now, many companies have focused their research on developing micro or miniaturized PEFC and DMFC for powering portable electronic devices and some of them have already demonstrated some interesting prototypes. Most of these prototypes are used to provide electricity for PDAs, cell phones and notebooks. 
In USA, MTI is one of the most aggressive companies working on the development of micro fuel cells used for portable electronic devices. It has recently made a lot of technique breakthroughs that led to a series of successful micro DMFC prototypes. Its first proof-of-concept DMFC system was demonstrated in the summer of 2001, which is shown in the top left of Fig. 3. This consisted of two micro fuel cells stacked together to provide greater overall power output while retaining a compact design. Later in October 2001, it presented an early prototype hybrid micro fuel cell system shown in top right of Fig. 3, which included a micro fuel cell, a fuel cartridge, a small hybridizing battery and the voltage regulation electronics. This system prototype demonstrated the ability to operate in any orientation, even upside down. The bottom left one in Fig. 3 was demonstrated in March 2002 and the power output was more than double that of the previous prototype. The bottom right one in Fig. 3 was finished in August 2002. This design included a replaceable methanol fuel cartridge, no pumps and worked in any orientation. The size of it was only 50 percent of that of the October 2001 prototype.

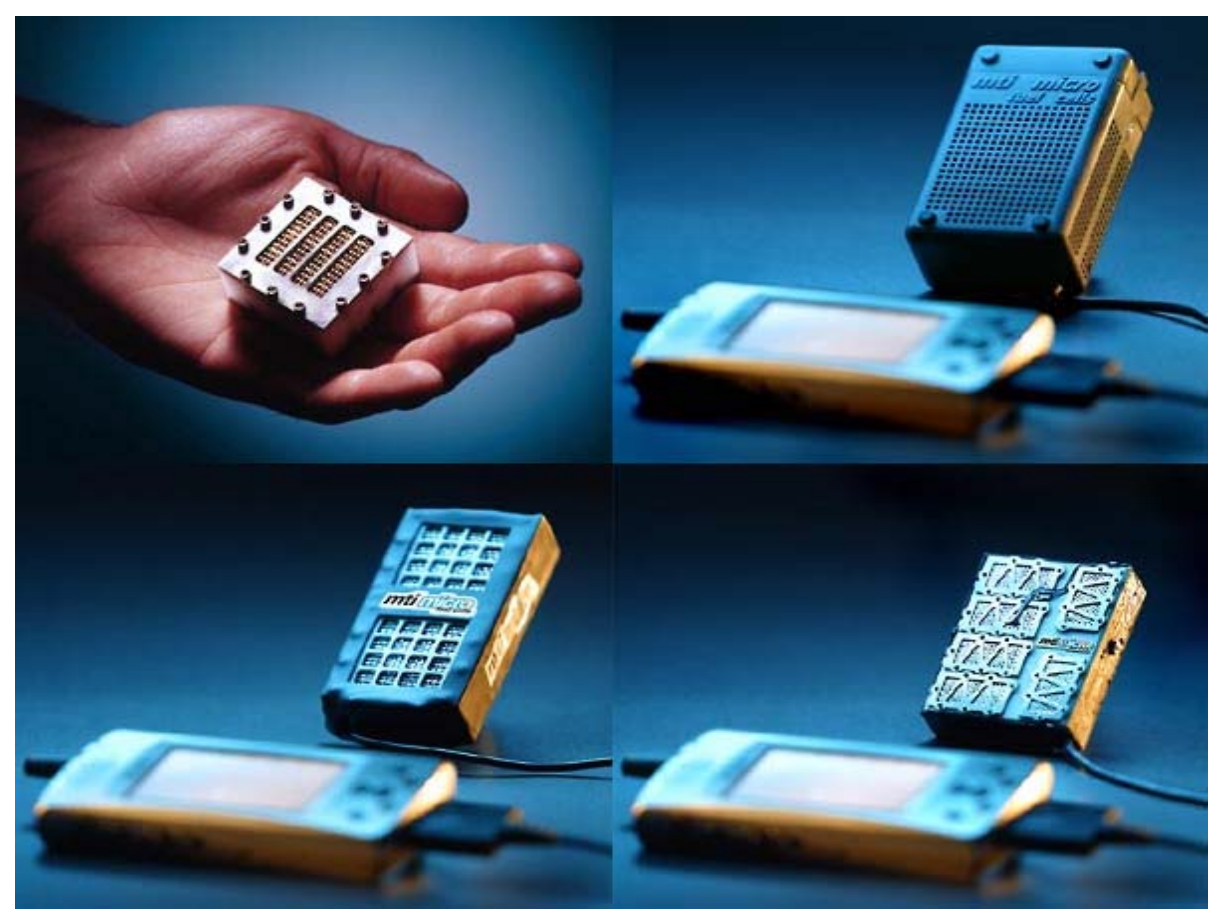

Figure 3 Prototypes of MTI micro fuel cell system 


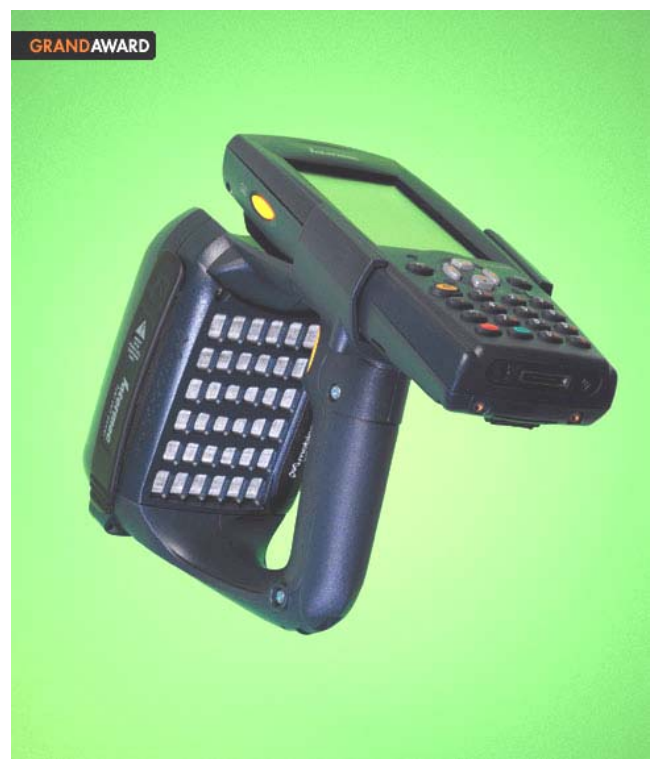

Figure 4 Intermac's newest RFID handheld reader using Mobion ${ }^{\mathrm{TM}}$ fuel cell technology

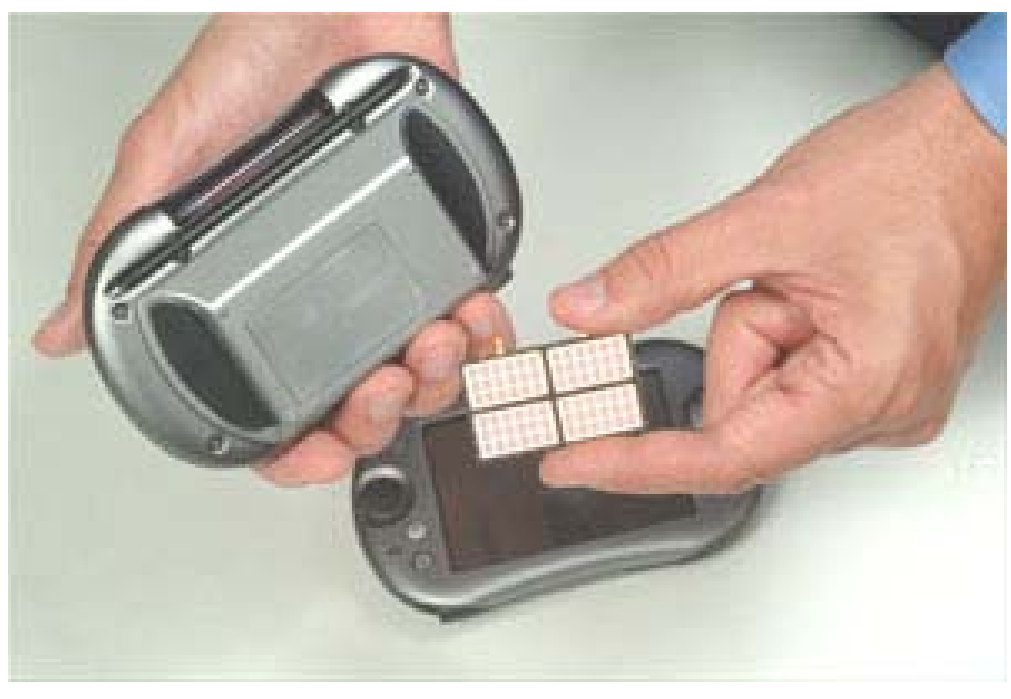

Figure 5 Two concept models incorporated with Mobion ${ }^{\mathrm{TM}}$ fuel cell technology

The latest technology developed by MTI is called Mobion ${ }^{\mathrm{TM}}$, which uses a different approach to solve the water management problem. It is claimed that this technology can achieve the water flow within the cell from cathode to anode without extra water management system. Therefore the $100 \%$ methanol can be directly fed into the anode that means more fuel can be carried and the whole system is more compact. Fig. 4 shows a radio-frequency identification reader (RFID) utilizing this new technology. It is reported that this device can run for 30 hours (8 hours if use 
conventional battery) before a new fuel cartridge is needed. The Mobion ${ }^{\mathrm{TM}}$ fuel cell can also be used for other portable electronic devices such as shown in Fig. 5: one is a handheld entertainment system, and the other is a PDA/smart phone.

Other American companies such as Motorola, PolyFuel, Neah Power Systems, and Medis Technologies LTD have also been working on micro fuel cell power systems. Fig. 6 shows a PDA powered by a Motorola DMFC prototype. The dimension of it is 2 inches by 2 inches. Electronic circuits integrated directly onto a ceramic substrate efficiently converts the output voltage of the planar fuel cell stack to the higher voltage required for driving the portable electronics. This prototype uses a highly simplified and miniaturized passive air-breathing design. Motorola lab is also exploring the possibility of utilizing reformed methanol fuel cell in which the methanol fuel is converted into a mixture of hydrogen and carbon dioxide before fed into the fuel cell stack.

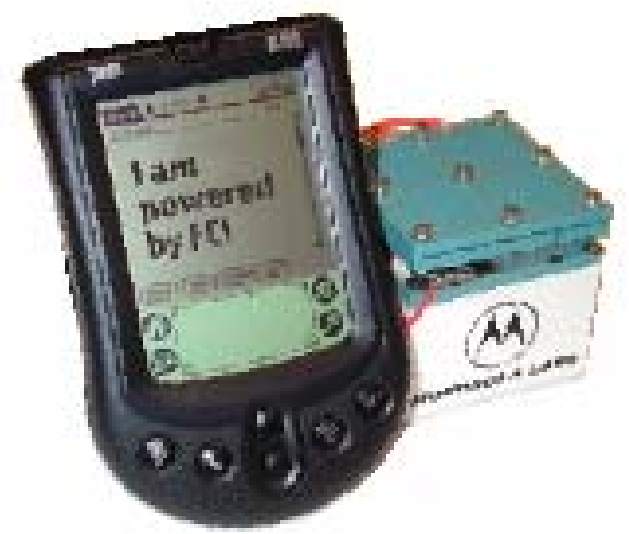

Figure 6 The Motorola DMFC prototype powering a PDA

Fig. 7 is one of the cell phone prototypes. It is a CA-based PolyFuel power system fitting on the back of a Nokia cell phone. Note that the honeycombed vents can let air in, water vapor out. PolyFuel uses a self-developed hydrocarbon membrane that is said to have less water flux and less methanol crossover than perfluorinated membranes. 


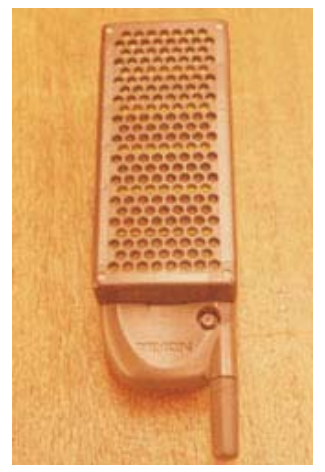

Figure 7 Prototype methanol micro fuel cell from Menlo Park

Instead of polymer membranes, Neah Power Systems utilizes porous silicon filled with liquid electrolyte (shown in Fig. 8) as the core of fuel cell. It is expected that the new structure can provide a much larger reaction area for electricity generation than conventional PEFC. Another advantage is that this design can use higher concentration of methanol fuel without methanol crossover. Taken together, a higher output power density can be achieved.

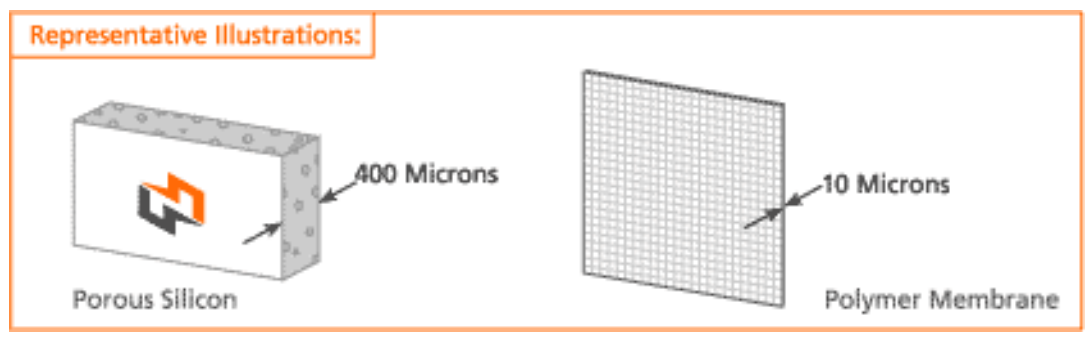

Figure 8 New electrolyte structure developed by Neah Power System
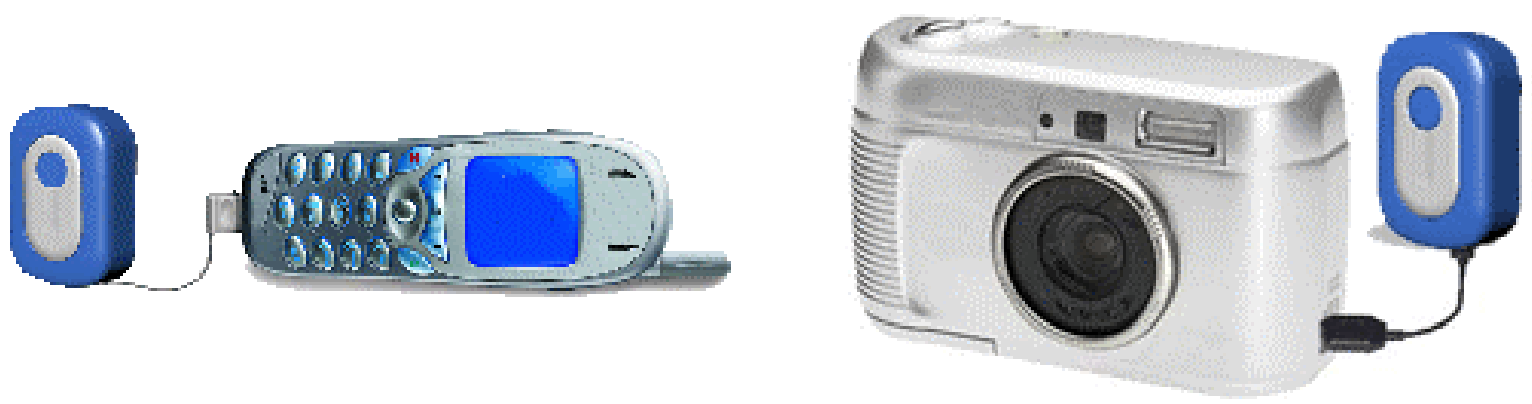

Figure 9 Medis Technologies LTD's power pack 
Fuel used in micro fuel cell power systems can also be different. Medis Technologies LTD developed a highly advanced liquid fuel, the basic components of which are borohydridealkaline solutions combined with alcohols. This kind of fuel has high levels of electrochemical activity. Unlike methanol, it is not flammable and not toxic. The electrolyte used is in liquid form. The power pack developed by Medis Technologies LTD has a very simple design and architecture, only consisting of an anode, a cathode, a chamber for the liquid electrolyte and a fuel chamber. The anticipated size of the power pack is 80 x 55 x 30 mm ( 3.2 x 2.2 x 1.2 inches) and has a weight of 120 grams empty and 200 grams fueled. Fig. 9 shows some of the applications of this power pack.

Many Japan companies are also very active in the research and development of micro fuel cell power systems. Casio’s fuel cell prototype for laptop applications uses methanol as fuel but not directly. A micro-reactor shown in the bottom right of Fig. 10 is used to reform methanol to hydrogen gas. It is reported that the reforming rate is very high, more than $98 \%$. Since the fuel fed into the fuel cell stack is hydrogen gas, the high output power density can be obtained while keeping a compact size. It is expected that a laptop PC with Casio fuel cell using methanol will be half in weight and provide about four times in the battery life time compared with that using a Lithium ion rechargeable battery. 


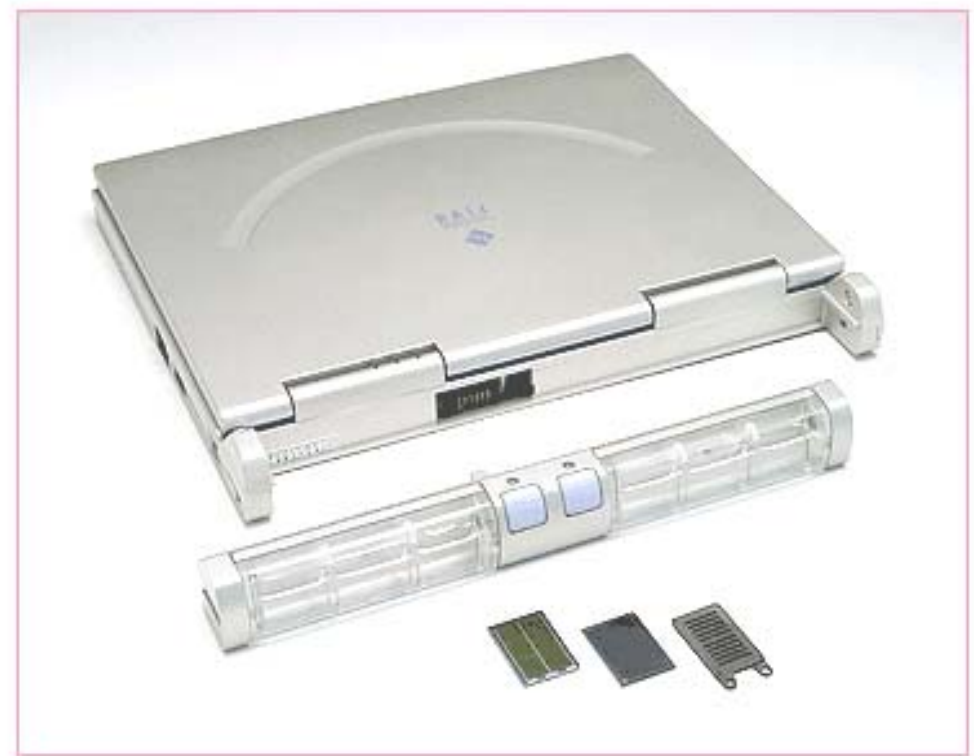

Commercial version(image)

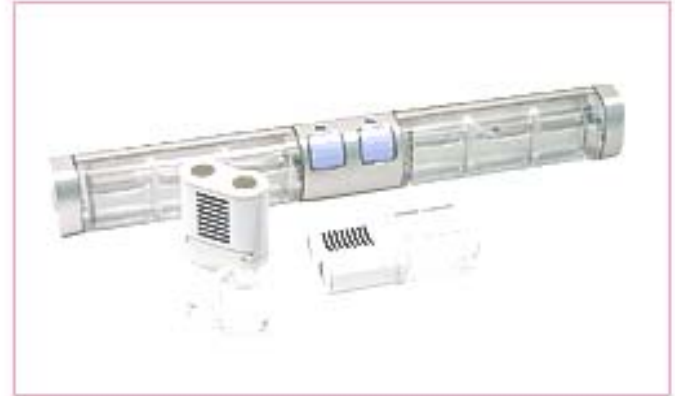

Upper component: laptop PC module Lower component: digital camera module (image)

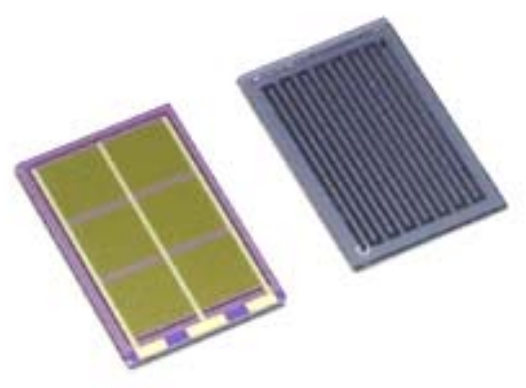

Micro-Reactor

(front and back)

Figure 10 Casio fuel cell prototypes

Fig. 11 shows a portable information terminal device powered by a methanol fuel cell. The whole system is developed by Hitachi. Shown in the top right is the fuel cartridge, which can be plugged into the cylinder chamber at the left side of the portable electronic device. The opening at the bottom of the device is for natural air ventilation. 


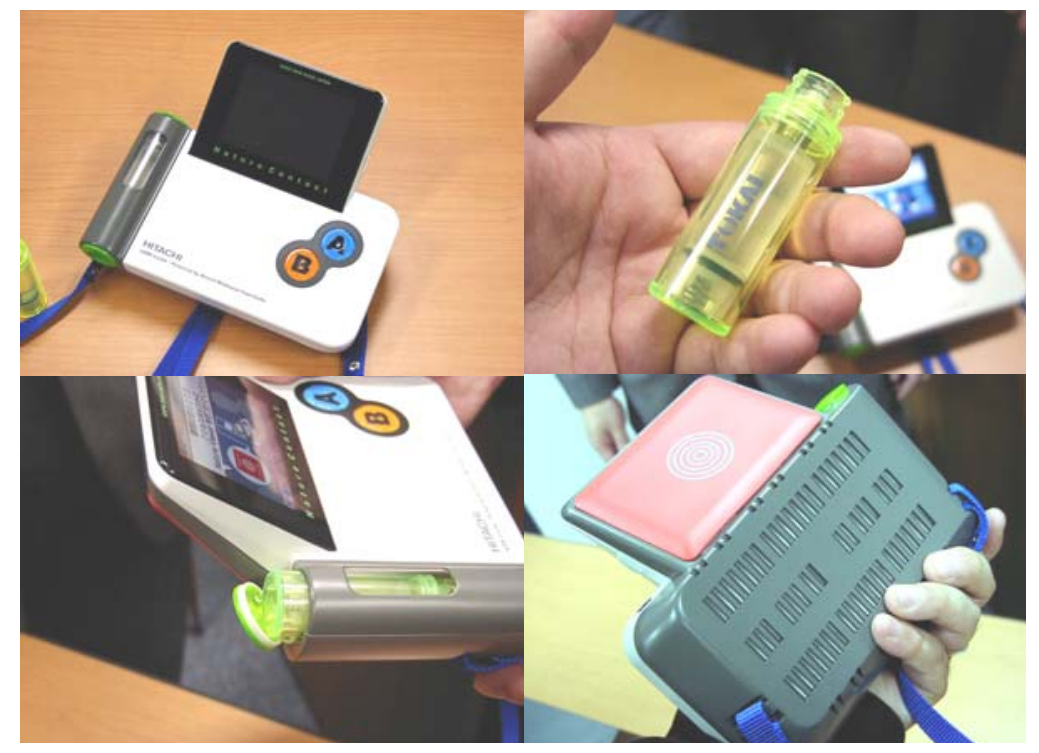

Figure 11 Hitachi fuel cell prototypes

The NEC's DMFC prototype shown in Fig. 12 employs carbon nanotubes as the basic building materials for its electrodes. The platinum particles deposited on carbon nanohorns are smaller than on ordinary substrates so the effective contact surface of the catalyst is increased. The power output density of the 2003's system was $50 \mathrm{~mW} / \mathrm{cm}^{2}$, and it is increased to $70 \mathrm{~mW} / \mathrm{cm}^{2}$ for the new model. NEC is also focusing on equipping the fuel cell with monitoring and management systems so that the power generation can be smooth and stable.
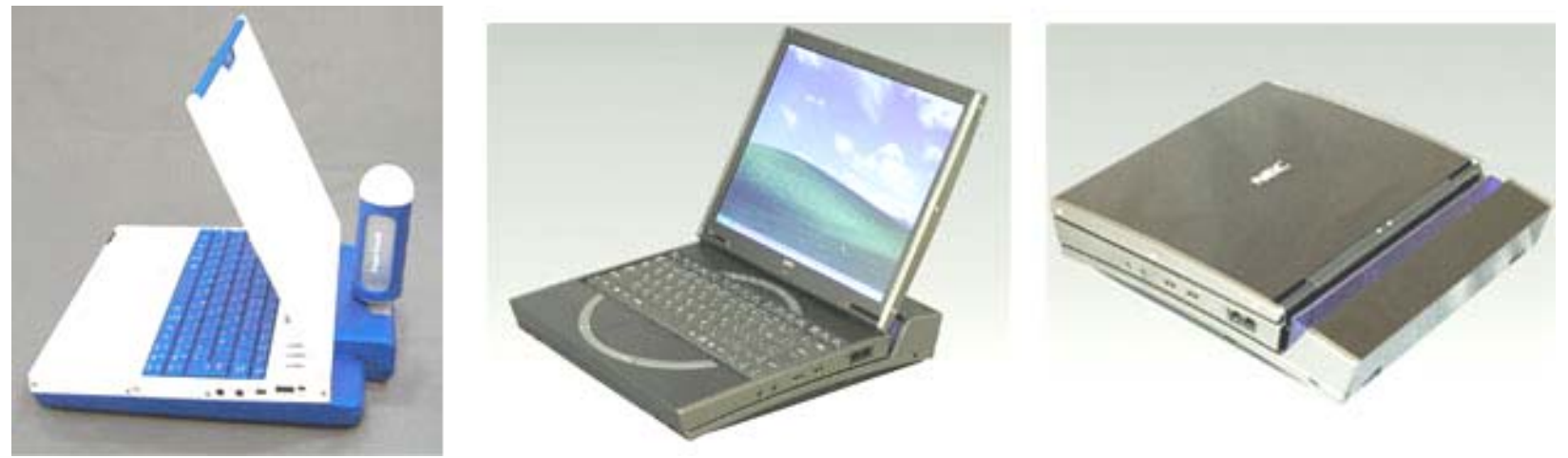

Figure 12 NEC fuel cell notebook prototypes from 2003 (left) to 2004 (center, right) 
NTT DoCoMo is developing a micro fuel cell prototype for its 3G cell phone handsets. The dimension of this prototype is 152 × 57 x $16 \mathrm{~mm}$ and it has a weight of 190 grams. The fuel used is $30 \%$ methanol and the power output is expected to be $5.4 \mathrm{~V}, 700 \mathrm{~mA}$.

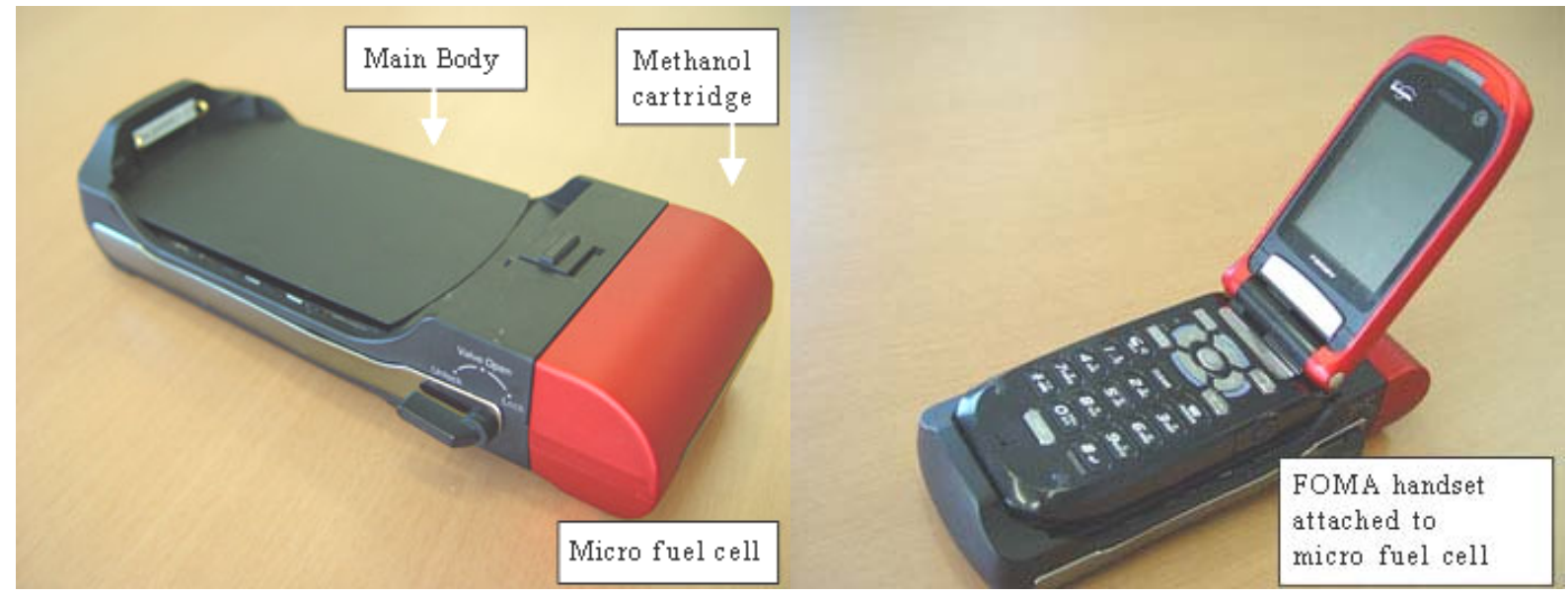

Figure 13 NTT DoCoMo 3G cell phone handset powered by micro fuel cell

Toshiba had developed micro fuel cell prototypes for both PDAs (Fig. 14) and laptops (Fig. 15). As shown in Fig. 14, Toshiba incorporates water recovery, recycling, carbon dioxide separation and onboard methanol/water mixing into their DMFC prototype to power a PDA therefore the whole system is quite large. To increase the current density, a motor-powered pump is used to speed up the methanol diffusion. Two tanks containing methanol and water separately are installed for internal dilution. The maximum output of this prototype is $8 \mathrm{~W}$ and the average output ranges from $3 \mathrm{~W}$ to $5 \mathrm{~W}$. Toshiba's micro fuel cell prototype for laptops is also very large (the back part attached to the laptop in Fig. 15) and it is necessary to shrink the dimension for real applications. 
(a) Driving a PDA

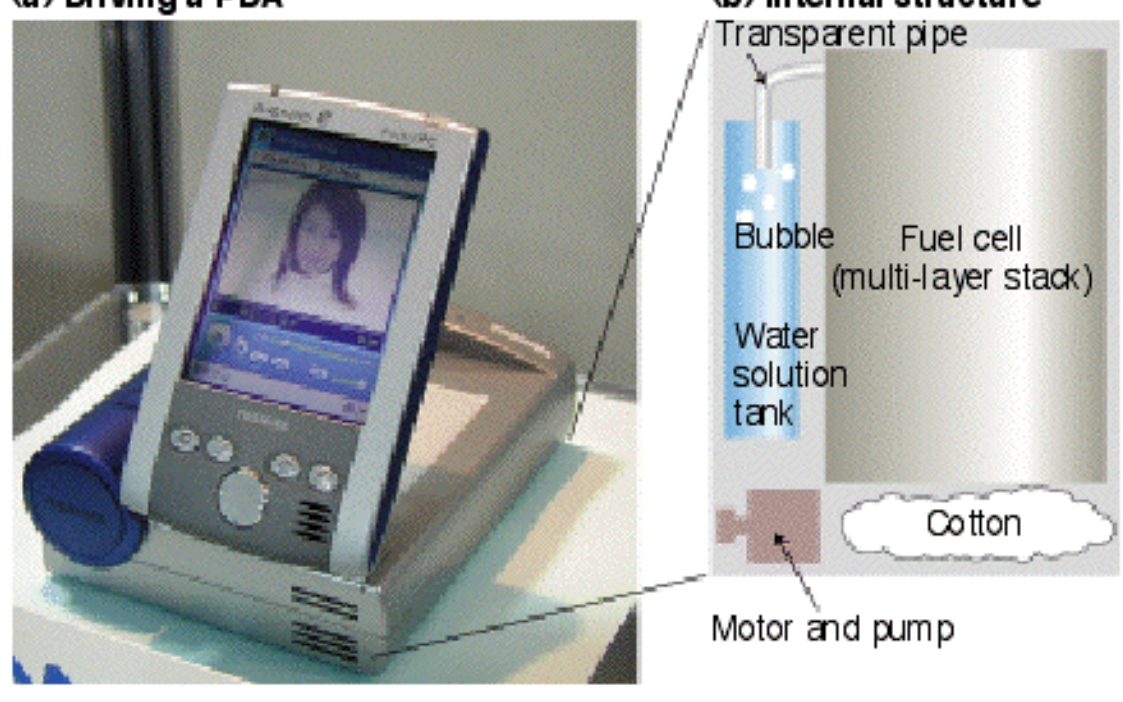

Figure 14 Toshiba prototypes fuel cell-powered PDA

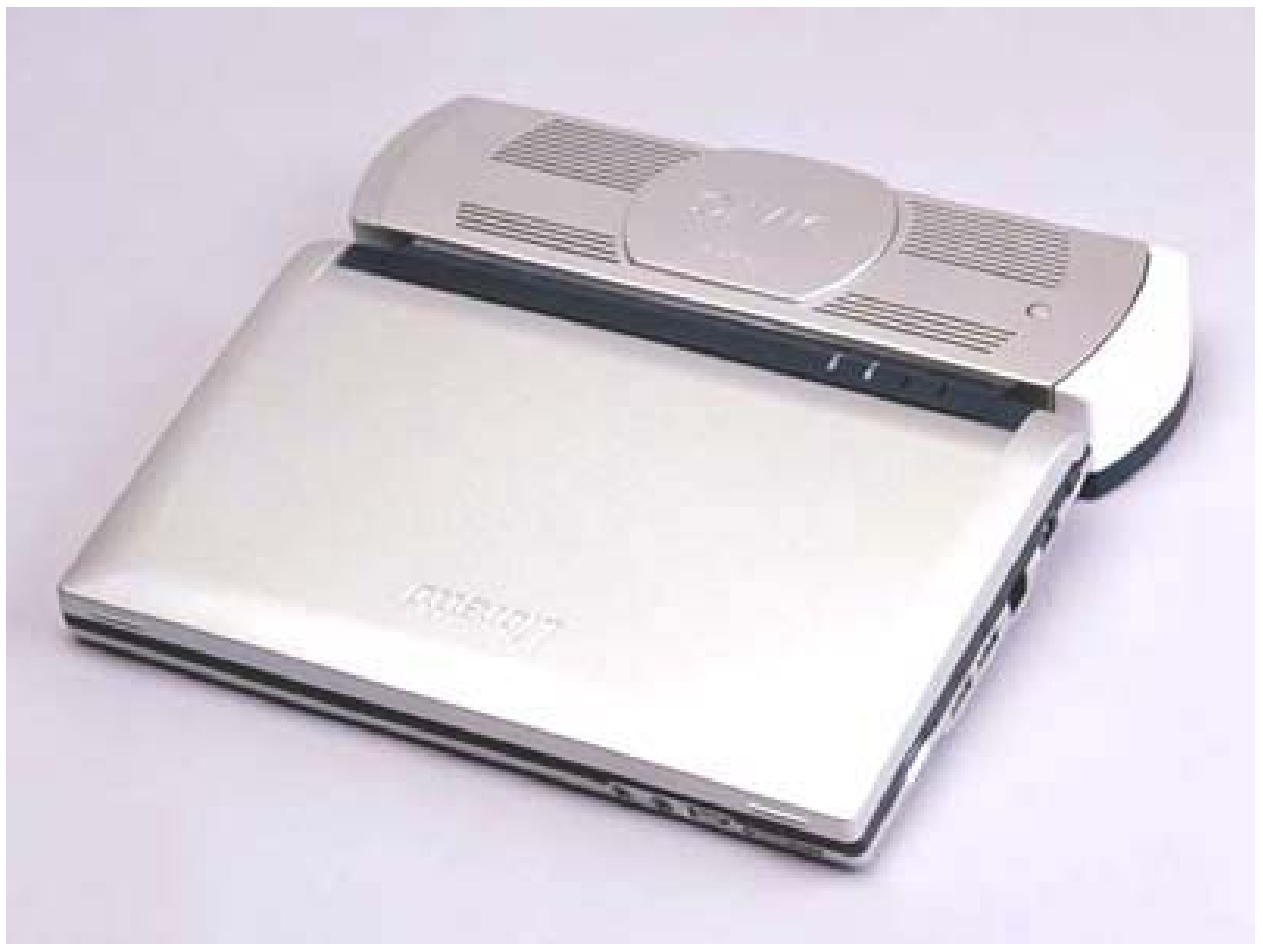

Figure 15 Toshiba prototypes fuel cell-powered laptop

In Europe, micro fuel cell power system for portable electronics is also in development. Most of the prototypes there are focused on PEMFC using hydrogen as fuel so the fuel storage problem remains to be solved. The prototype shown in Fig. 16 is a miniaturized hydrogen PEFC system 
developed by a German company. A small metal hydride tank is used to supply hydrogen to the fuel cell stack. The whole fuel cell stack looks like a perforated cylinder so the air can flow into the cells stack through the mesh-like holes on the side. Obviously this is not a compact design and the cost of the hydrogen storage tank makes the whole system too expensive for ordinary users.

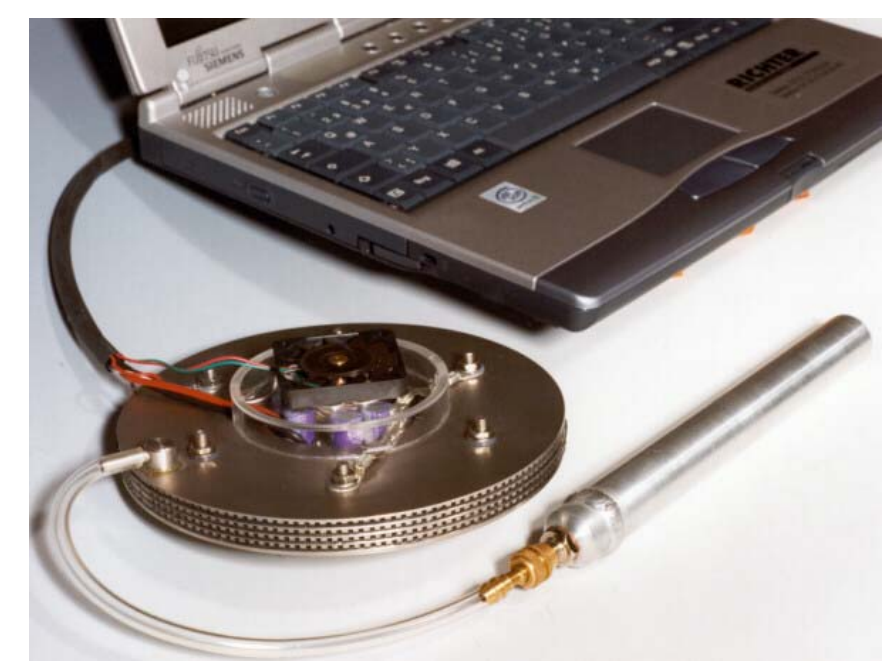

Figure 16 Fraunhofer ISE's micro-fuel cell powering a laptop

From these prototypes introduced above, it can be found that the micro or miniaturized DMFC is the most widely used technology for powering portable electronics. This is because PEFC suffers from the storage problem of hydrogen although it usually has a better performance than DMFC. Using liquid methanol solution as fuel directly, DMFC is therefore much more straightforward and attractive for commercial applications. Many researchers [2, 3] have discussed the benefits and challenges utilizing such a direct-fuelled system. Also for commercial portable applications, the preferred operation conditions for micro or miniaturized DMFC are ambient pressure and temperature, air breathing (air supply by natural convection) cathodes and as few peripheral devices as possible (pumps, valves, fans, etc.) [4]. 
To optimize a micro or miniaturized DMFC power system, there are many engineering issues to be considered, which include materials selection, fabrication method, thermal management, water management, methanol crossover, fuel delivery, oxidant supply, power conditioning and etc. Many of them have already been addressed in academic publications.

Most commonly, various micro fuel cell designs are fabricated on silicon substrate using advanced silicon-processing and thin film technology such as photolithographic pattering, dry and wet etching, chemical and physical vapor deposition, spin-cast coating [5-8]. Most of these micro fuel cells were tested using hydrogen but could use methanol as fuel with further adaptation. The designs can be either monolithic [5, 6] or bilayer [6-8]. It was found that bilayer design had a much better performance than monolithic design [6]. Also the performance of miniaturized fuel cells was generally lower than that of conventional fuel cells and high cell resistance was suggested to be one of the major reasons [6]. This problem can be partially solved by sputtering a $\mathrm{Cu} / \mathrm{Au}$ composite layer on the top of the silicon wafer to reduce its resistance as a current collector [8].

Other than silicon, other substrates can also be used to fabricate micro fuel cells. It is reported that prototypes of self-breathing PEFC with a size of $1 \mathrm{~cm} \times 1 \mathrm{~cm}$ and a thickness of $200 \mu \mathrm{m}$ were fabricated on stainless steel foil and the stable operation was achieved at $80 \mathrm{~mW} / \mathrm{cm}^{2}$ under ambient condition with dry hydrogen fuel [9]. Another micro fuel cell was fabricated on a polydimethylsiloxane (PDMS) substrate and operated at room temperature with a maximum power density of about $700 \mu \mathrm{W} / \mathrm{cm}^{2}$ with hydrogen as fuel [10].

Comparing with hydrogen, the electrochemical reactivity of methanol is more sluggish. One way to solve this problem is to use a micro methanol fuel processor that can convert methanol to hydrogen, carbon dioxide and remainder carbon monoxide. For a $15 \mathrm{~W}$ portable fuel cell power 
supply, the thermal efficiency of the fuel processor was able to reach $60 \%$ and the efficiency of the whole system was $29 \%$ [11]. For another sub-watt application, a 33\% thermal efficiency of the fuel processor was achieved [12]. Certain progress has been made on micro fuel professors. However, the design with a micro methanol fuel processor is less favorable because it will add to the complexity and cost of the whole system, also with extra energy consumption.

Other methods that can improve the performance of micro fuel cells have also been investigated. A new "flip-flop" interconnection between micro fuel cell arrays was presented to simplify the membrane processing, interconnection design and decrease connection resistance [13]. The factor of scaling behavior of different flow patterns (interdigitated, serpentine and spiral interdigitated) was studied using CFD [14]. Different membranes such as acid loaded porous silicon [15] were fabricated and tested. The addition of sulfuric acid with methanol fuel can also improve the cell performance by increasing the conductivity of fuel solution [16].

Another issue has to be aware about micro or miniaturized DMFC is the separation and removal of carbon dioxide generated at the anode side. In the worst case, these carbon dioxide bubbles can cling to the reaction surface or block the micro flow channels. Either way, the performance of the micro fuel cells will deteriorate very quickly. Using deep reactive ion etching (DRIE) technique, a passive gas-liquid separation system for micro DMFC was presented [17]. Both gas and liquid separators consisted of staggered arrays of etched-through holes. The gas separator was coated with a thin layer of hydrophobic polymer while the liquid separator was simply made of an array of holes on a hydrophilic silicon wafer. Successful gas-liquid separation was observed at liquid pressures between 14.2 and $22.7 \mathrm{cmH}_{2} \mathrm{O}$, liquid flow rates between 0.705 and $1.786 \mathrm{cc} / \mathrm{min}$, and $\mathrm{CO}_{2}$ flow rates between 0.15160 and $0.20435 \mathrm{cc} / \mathrm{min}$. 
As mentioned above, there are many research issues to be solved for the development of micro or miniaturized DMFC power systems. In this thesis, the main focus will be the fuel delivery problem. There are several requirements for the fuel delivery system. First of all, it should be able to provide sufficient flow rate for the fuel cell reaction. Second, the power consumption of it should be as small as possible so more net output power can be obtained. Also it should be easy to control so it can match well with the fluctuation of the outer load. Last the fabrication and the system integration should be simple, economical, and the size of it should be as small as possible. For medium and large size DMFC, conventional pumps can be used to drive the fuel flowing through the fuel cell. For micro or miniaturized DMFC, there are several options for fuel delivery and micropump is one of them.

\subsection{MICROPUMP}

\subsubsection{Pump basics}

Pump is one of the earliest inventions in human civilization and the earliest pumps can be traced back to the undershoot-bucket waterwheels that can carry water into a collecting rough as the force of stream is pushing the wheels rotating (center of Fig. 17). Another early pump, Archimedes' screw pump (Fig. 18) is still being utilized to transport liquid-solid mixtures nowadays. With the time passing by, numerous pumps with different dimensions have been developed based on various pumping mechanisms for different applications. It is estimated that the pump is probably the second most common machine in use, exceeded only by the electric motor [18]. 

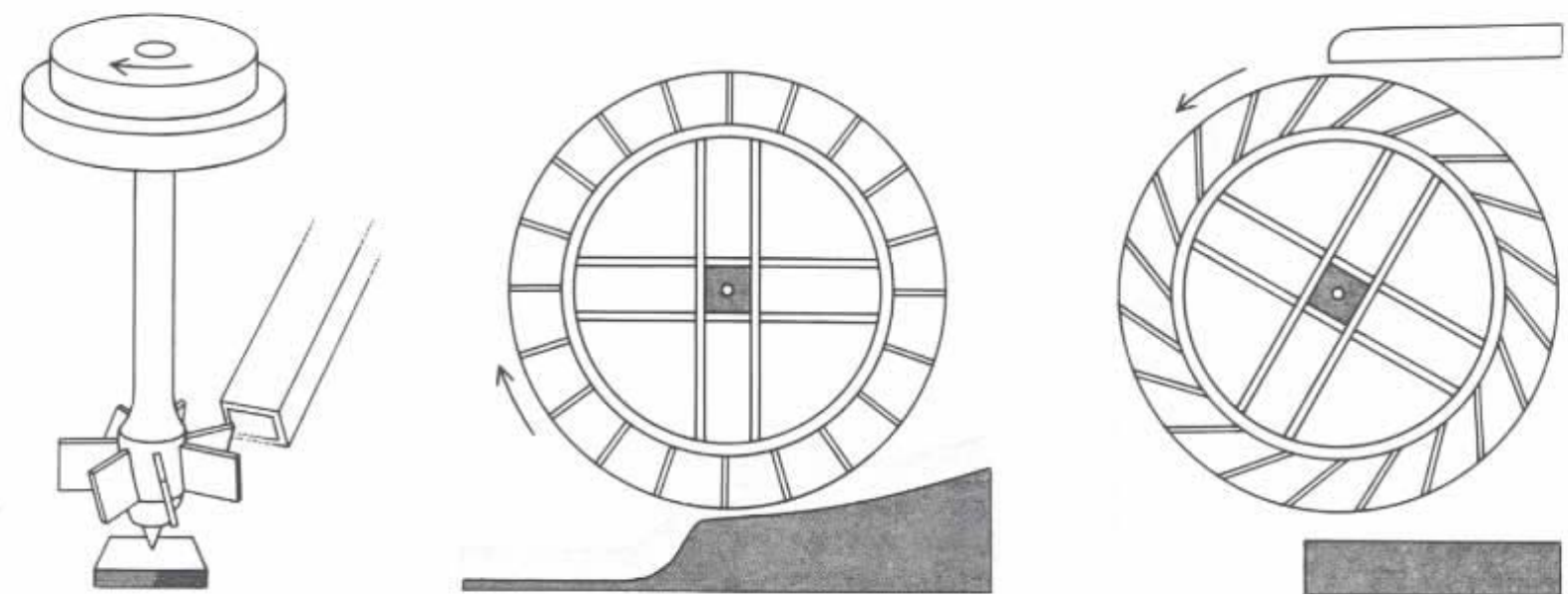

Figure 17 Illustration of waterwheels designs

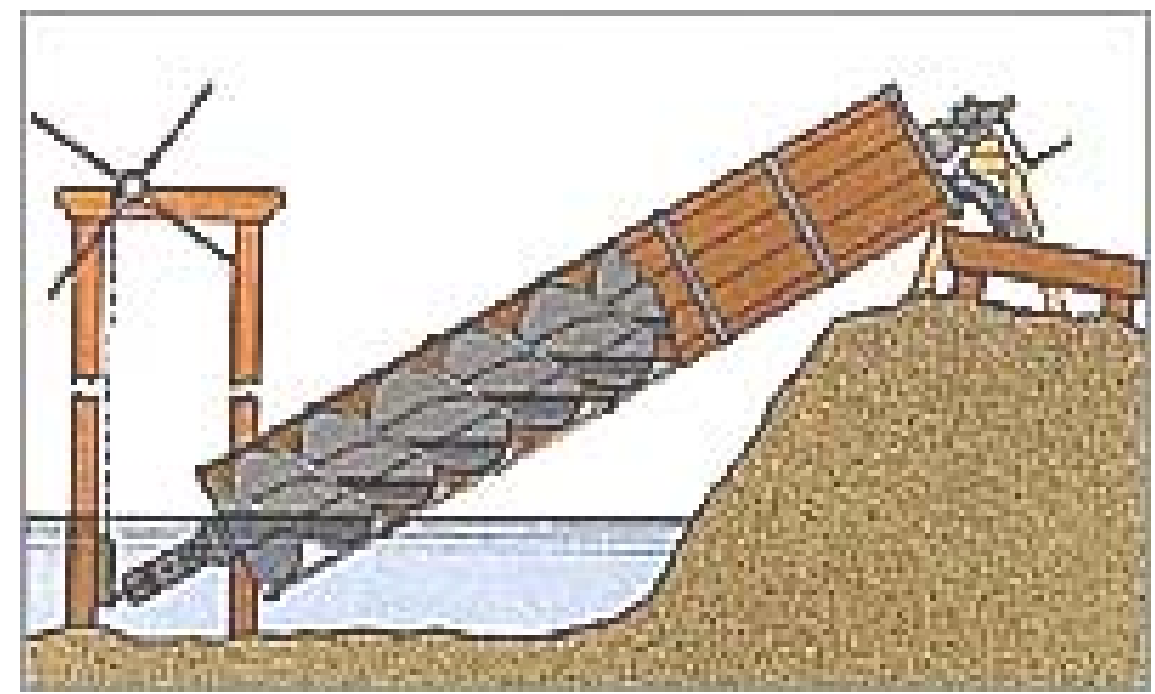

Figure 18 Illustration of Archimedes' screw pump

There are many methods that can be used to classify pumps such as the applications they served, the materials being used for manufacturing, and the liquids in transportation. All these classifications can be limited and overlap with each other. A more general classification based on the pumping principle is used here and according to this method, pumps can be divided into two groups: dynamic pumps and displacement pumps. In dynamic pumps, the momentum energy is continuously added to the fluid so that high fluid velocities can be obtained within the pumps. Since these velocities are higher than those at the outlet, this velocity difference can result in a 
pressure difference. This pressure difference is then pushing fluid moving forward. Dynamic pumps can be further divided into centrifugal pumps (including axial flow, mixed flow or radial flow, and peripheral) and other special-effect pumps (including jet, gas lift, hydraulic ram and electromagnetic). In displacement pumps, the energy is periodically added to the fluid by applying force to the movable boundaries of an enclosed fluid volume. As a result, the pressure of the fluid varies periodically while the inlet and outlet open alternately. Therefore the fluid is being transported. A classic example of displacement pumps is human heart that can pump blood throughout the whole body. Displacement pumps can be further divided into reciprocating pumps (including diaphragm, piston or plunger) and rotary pumps (including vane, screw, gear, flexible membrane and etc.).

Generally, dynamic pumps can supply a higher flow rate and the output flow is much steadier, while displacement pumps can provide a high-pressure rise and have less difficulties in the delivery of high-viscosity fluids.

\subsubsection{Micropump history}

The research on micropumps initially emerged at Stanford University in 1980 [19]. Since then micropumps have received a lot of attention and have played an important role in the development of microfluidics systems. The applications of micropumps include chemical analysis systems, microdosage systems, ink jet printers and other microelectromechanical systems (MEMS) that require microliquid handling.

During the last several decades, various designs of micropumps made of different materials and based on different pumping mechanisms have been presented. As often cited, J. G. Smits [20] developed one of the earliest micropumps and published it in 1990. As shown in Fig. 19, this 
micropump was fabricated on a 2-inch silicon wafer bonded with 2 glass plates on each side. And three piezoelectric disks were glued on the thinner glass plate over the area where the inlet and outlet orifices were located. When these three piezoelectric elements were actuated one by one to form a peristaltic motion, a certain flow rate from inlet to outlet was established. Water was the working fluid. The pump was able to obtain a flow rate of $100 \mu \mathrm{l} / \mathrm{min}$ at zero backpressure and a maximum pump pressure of $60 \mathrm{~cm} \mathrm{H}_{2} \mathrm{O}$ at zero flow rate. The maximum pumping speed was reached at a driving frequency of $15 \mathrm{~Hz}$ and no pumping effect was observed at driving frequencies higher than $50 \mathrm{~Hz}$. The self-priming ability of this pump was very poor and the outgassing of water was necessary for the normal operation. In spite of these drawbacks and low performance, the basic principle of this micropump proved to be solid and has been adopted into other new micropump designs till now.

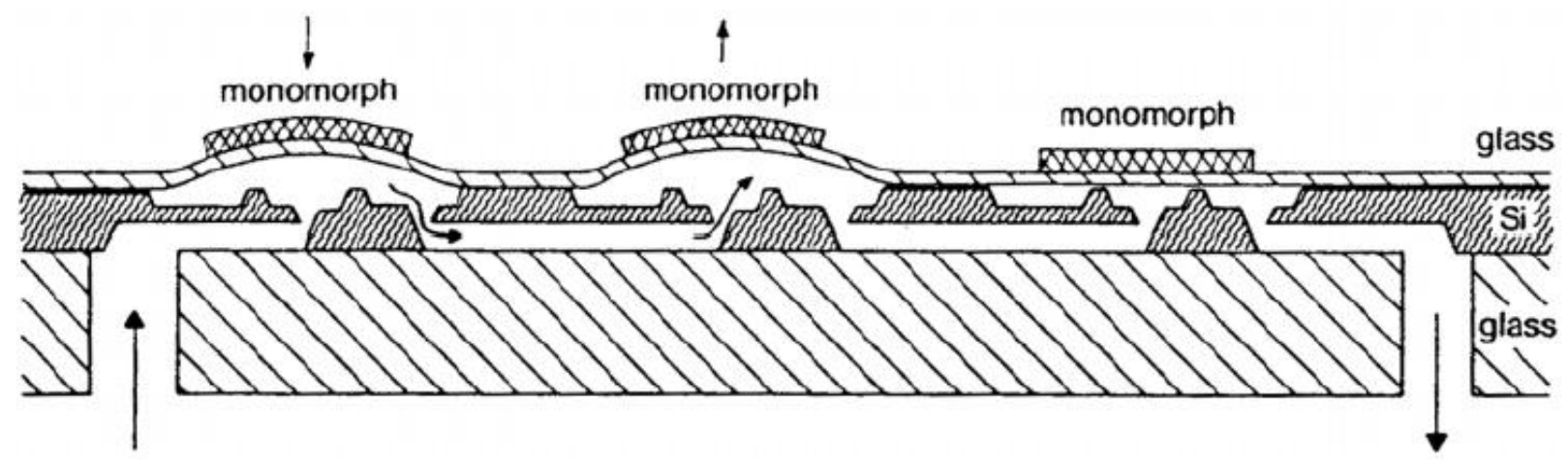

Figure 19 The piezoelectric micropump developed by J. G. Smits 


\subsubsection{Micropump types}

According to the classification method mentioned above, micropump can also be divided into two groups: dynamic micropumps and displacement micropumps. An extensive review about different types of micropumps has been presented recently [21] and some of its content related to this research is introduced briefly below.

\section{Dynamic micropumps}

There are great difficulties and limitations in scaling down the conventional centrifugal pumps. Other alternatives to pump fluid at micro scale have been explored including electromagnetic effects and acoustic effects. Based on the interactions between the fluid and the electromagnetic field, electrohydrodynamic, electroosmotic and magnetohydrodynamic micropumps are developed.

\section{Electrohydrodynamic (EHD) micropumps}

The principle of electrohydrodynamic (EHD) micropumps is to utilize the effects of electrostatic forces with ions in dielectric fluids. For the EHD micropumps using the Coulomb force acting on free charges, the operation of them requires the existence of space charge in a dielectric fluid. For the EHD micropumps using the polarization force rather than the Coulomb force, the flow is generated through electric field interactions with dipoles in a polarized medium. One of the possible applications of EHD micropumps is microelectronics cooling.

\section{Electroosmotic (EO) micropumps}

When a fluid comes in contact with a solid, certain surface charge will develop spontaneously. Also a so-called electrical double layer (EDL) will form due to the shielding of this surface charge by the bulk liquid counter-ions. In electroosmotic (EO) micropumps, an electric field 
parallel to the wall is applied so that some portions of the counter-ions in EDL can be set into motion. The mobile ions then drag bulk liquid in the direction of the electric force. The performance of EO micropumps depends on (i) the magnitude of the applied electric filed and applied voltage, (ii) the cross-sectional dimensions of the structure in which flow is generated, (iii) the surface charge density of the solid surface that is in contact with the working fluid and (iv) ion density and $\mathrm{pH}$ of the working fluid. EO flows has been used in a wide range of applications, especially in the area of chemical and biological analysis.

Magnetohydrodynamic (MHD) micropumps

In magnetohydrodynamic (MHD) micropumps, a magnetic field is applied to the aqueous solutions with current-carrying ions so that a Lorentz force can be generated to induce flow. The performance of MHD micropumps is limited by (i) the magnetic flux density, (ii) the scaling effect that makes miniaturization difficult, and (iii) the thermal effect that limits current density.

\section{Other dynamic micropumps}

The dynamic micropumps based on the flow induce by flexural waves propagating through a membrane in contact with the fluid have been reported recently. Piezoelectric actuators are used to generate the ultrasonic flexural waves in these micropumps. Other dynamic micropumps based on thermal transpiration have also been presented.

\section{Displacement micropumps}

Displacement micropumps can be further divided into reciprocating displacement, aperiodic displacement and rotary displacement micropumps.

\section{Reciprocating displacement micropumps}

Of all the micropumps, reciprocating displacement micropumps that use movable boundary to push the working fluid periodically attract the most research attention. In these micropumps, the 
movable boundary is often a deformable plate - the pump diaphragm - with fixed edges. The pump diaphragm can be made of silicon, glass, plastic and metal. Besides the pump diaphragm, other basic components include a pump chamber, an actuator mechanism or driver and flow directing elements (valves, nozzle/diffuser and etc.). In operation, the actuator drives the pump diaphragm to increase and decrease the volume of the pump chamber periodically. When the pump chamber is in expansion, the fluid is drawn in. When the pump chamber is in contraction, the fluid is forced out. The flow directing elements are carefully designed and regulated so that a net flow from inlet to outlet can be obtained during one circle of expansion and contraction.

There exists a wide range of designs in pump chambers, actuation mechanisms and flow directing elements for reciprocating displacement micropumps. Most micropumps only have one single pump chamber but some have multiple pump chambers connected in series or in parallel. The micropump shown in Fig. 20 only has one pump chamber and two passive check valves. And the micropump shown in Fig. 19 has three pump chambers connected in series and no valve is required.

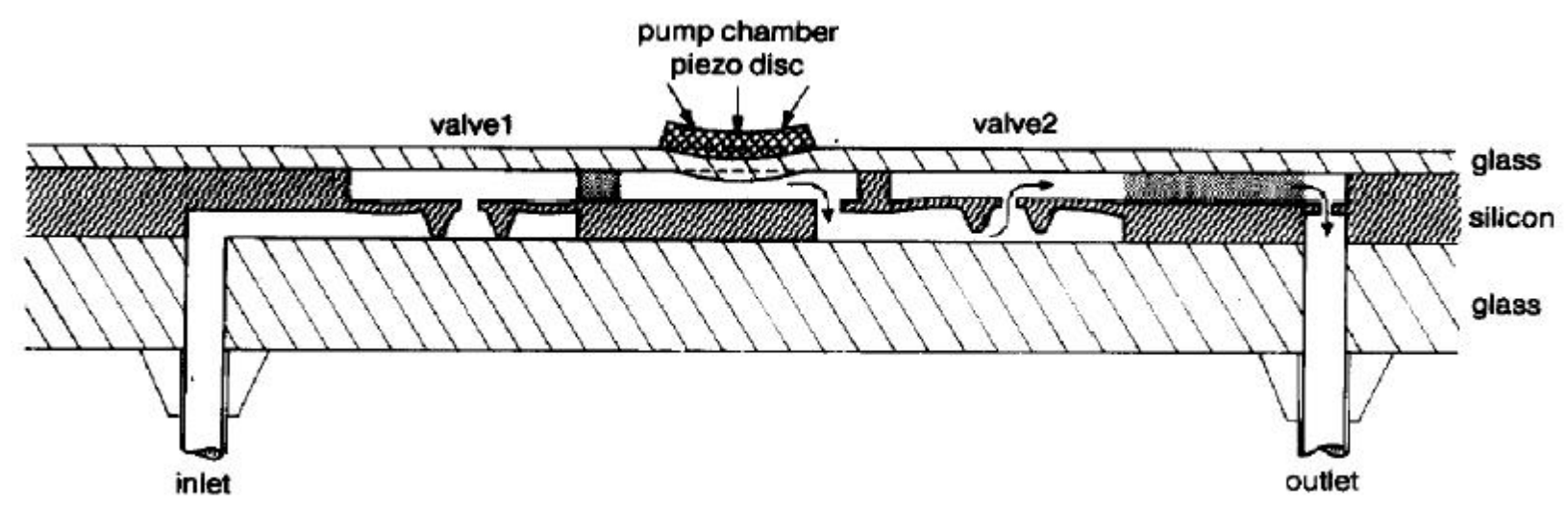

Figure 20 Reciprocating displacement micropump with single pump chamber [22]

The actuation mechanisms used in reciprocating micropumps include piezoelectric effect, thermopneumatic effect, electrostatic effect, and pneumatic effect. How these mechanisms work in micropumps is shown in Fig. 21. In (a), the micropump is driven by a piezoelectric bending 
mode actuator. The bottom surface of the piezoelectric disk is bonded to the pump diaphragm while the top surface is free. When applying an electric field in the polarization direction, the diaphragm deflects due to the bending moment introduced by radial strain in the piezoelectric disk. Different in (b), the micropump is driven by a piezoelectric thickness mode actuator. The bottom surface of the piezoelectric disk is also bonded to the pump diaphragm but the top surface is bonded to a rigid frame. When applying an electric field in the polarization direction, the diaphragm deflects mainly as a result of the axial strain in the piezoelectric disk. The thermopneumatic mechanism is used in (c). When the thin film heater heats the driver working fluid inside the second chamber on top of the pump chamber, the heated fluid will expand and therefore exert pressure on the diaphragm. In (d), the electrostatic driver is used to pump the fluid. There are two parallel electrodes in this micropump: One is bonded to the pump diaphragm and the other is bonded to a rigid frame. The diaphragm can deflect by applying an electric field between these two electrodes. Last in (e), the pressure above the pump diaphragm varies periodically by alternately pressurizing and venting the gas in a second chamber above the pump chamber. This is called an external pneumatic driver. 

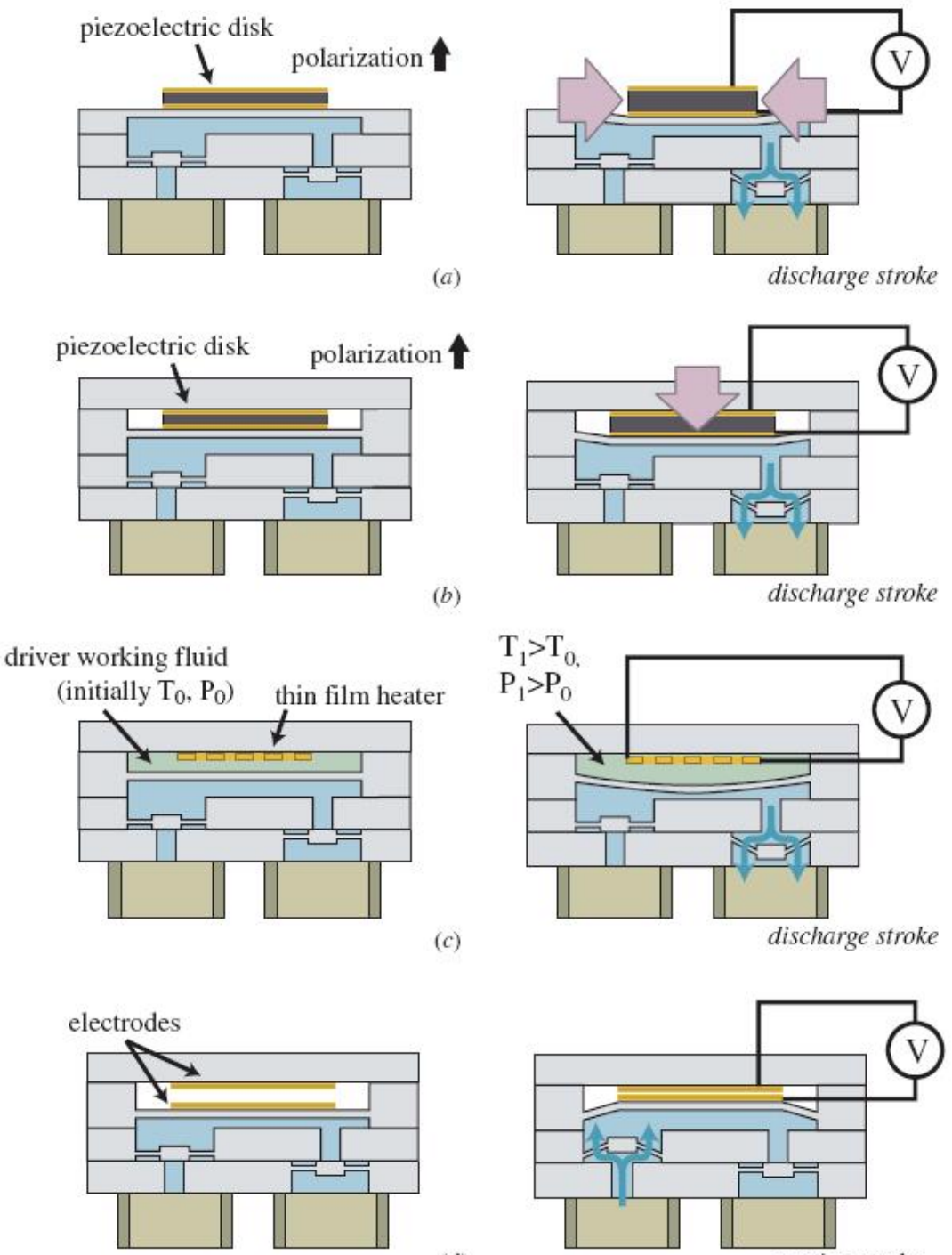

(d)
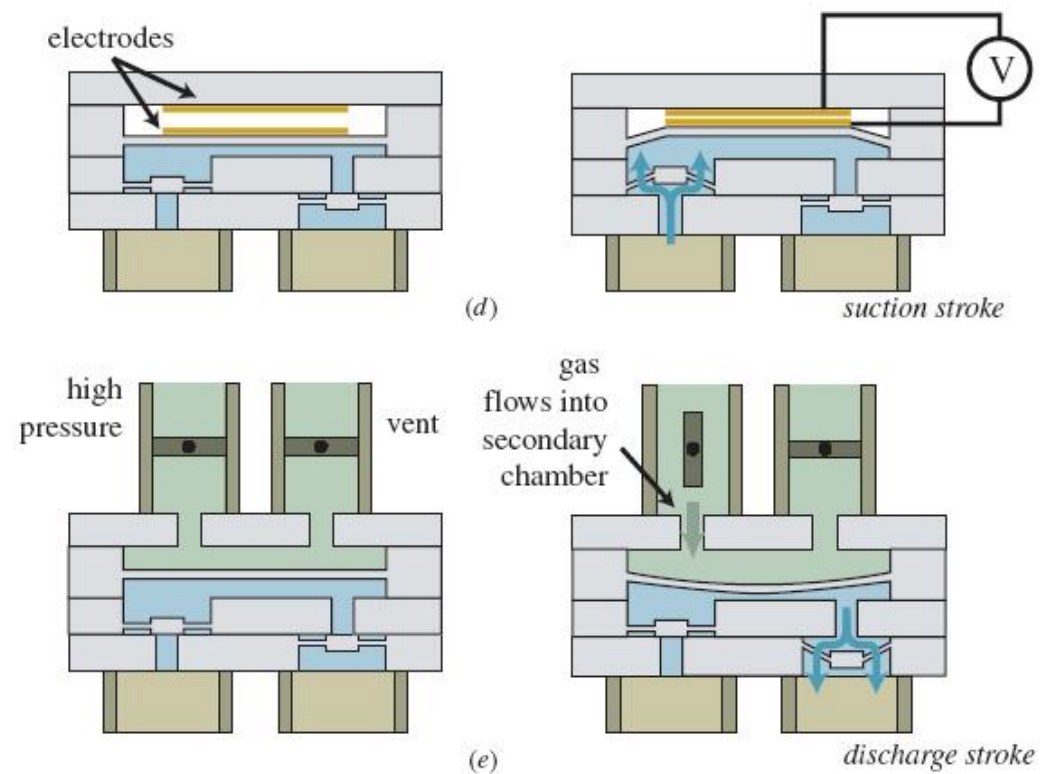

Figure 21 Reciprocating displacement micropumps with different actuation mechanisms 
The flow directing elements are very important for the micropump operation and several different designs have been presented. In earlier research, passive check valves were often used (Fig. 20 and Fig. 21). To eliminate the wear and fatigue of valves and reduce clogging, valve-less designs started to attract more research attention. The first piezoelectric valve-less micropump was introduced in 1993 [23]. As shown in Fig. 22, nozzle/diffuse elements were used to rectify the flow direction in this design. The opening angles of these nozzle/diffuser elements were small, normally less than $20^{\circ}$ and the diffuser direction was the positive flow direction. A different type of nozzle/diffuser elements fabricated in silicon using anisotropic wet etching was presented in 1995 [24, 25]. As shown in Fig. 23, the opening angle of this design was $70.5^{\circ}$ and the positive flow direction was in the converging wall direction. Besides nozzle/diffuser elements, another flow directing element called valvular conduit was also used in micropumps and it was reported that this design had higher volumetric efficiency than nozzle/diffuser elements [26]. The photo of this valvular conduit is shown in Fig. 24.

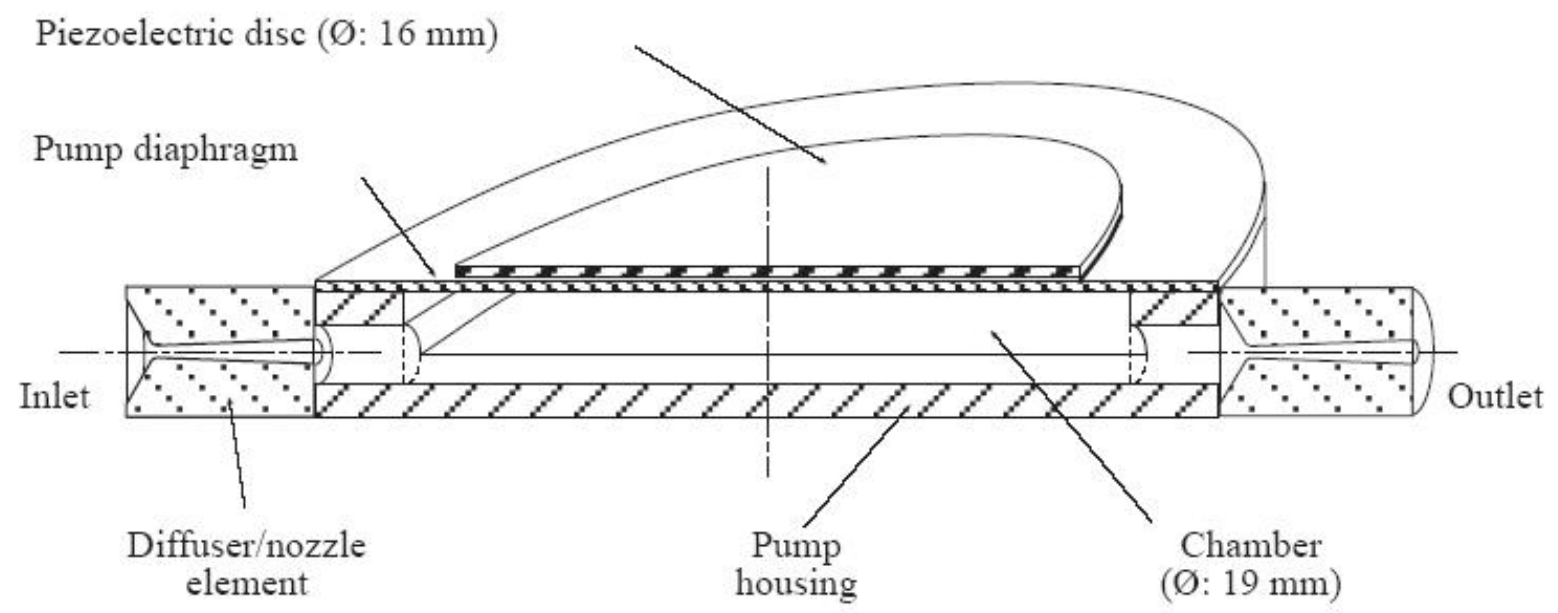

Figure 22 Piezoelectric valve-less micropump with conical nozzle/diffuser elements 


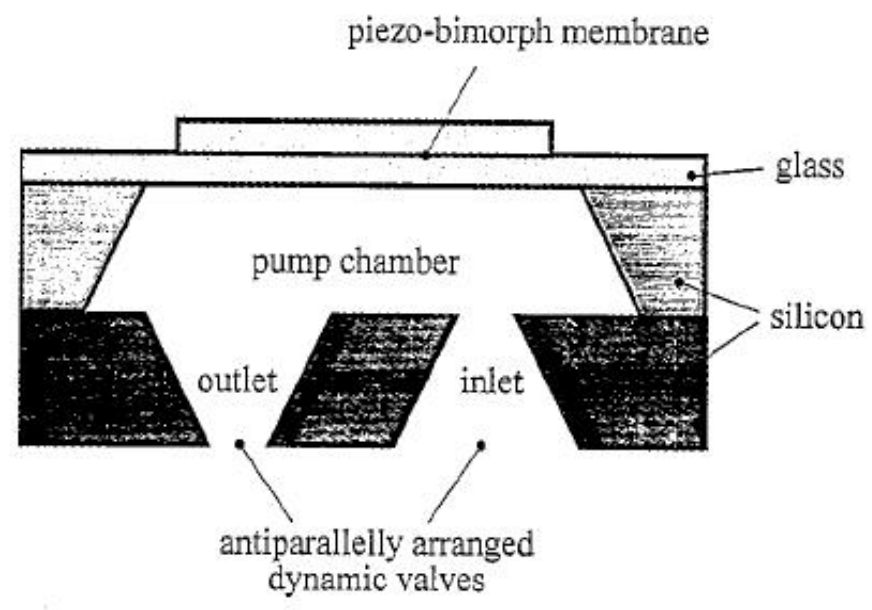

Figure 23 Piezoelectric valve-less micropump with pyramidic nozzle/diffuser elements

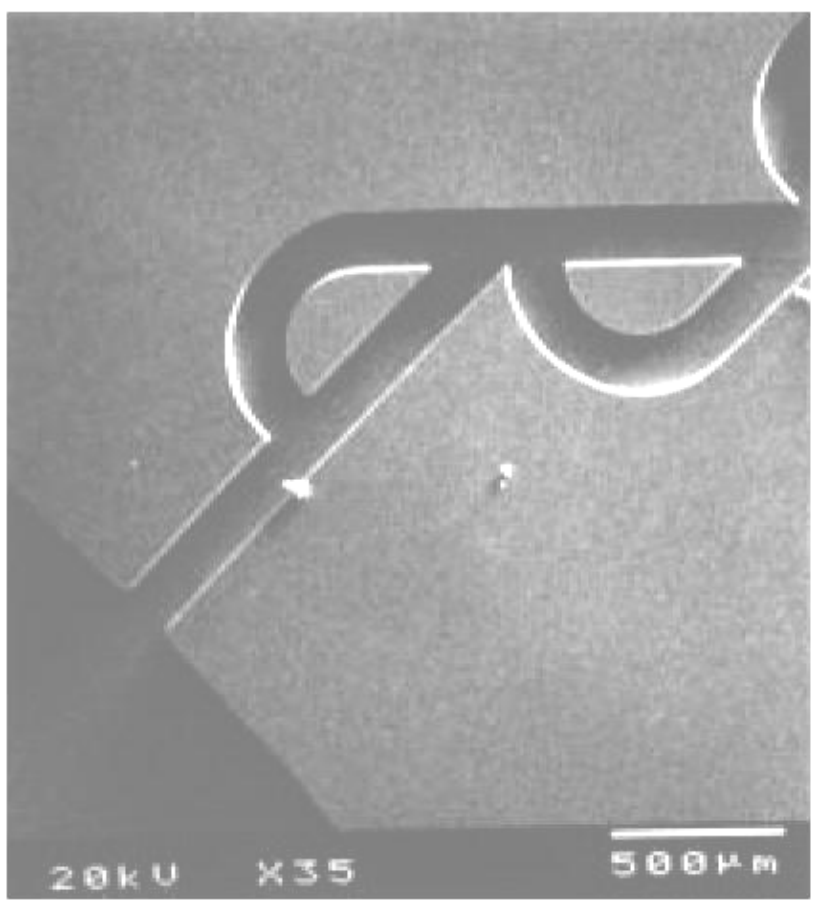

Figure 24 Valvular circuit 
In summary, reciprocating displacement micropumps have a very wide variety of chamber configurations, actuation mechanisms and flow directing element types. Accordingly the performance of them also covers a broad range: the flow rate ranges from several $\mu \mathrm{l} / \mathrm{min}$ to tens of $\mathrm{ml} / \mathrm{min}$, the pressure head ranges from several hundred $\mathrm{Pa}$ to hundreds of $\mathrm{kPa}$ and there is almost no limitation for working fluids.

Aperiodic displacement micropumps

Aperiodic displacement micropumps also drive the working fluid by the deformation of movable surface or boundary, but the movement of such surface or boundary is neither reciprocating nor periodic. Usually, this type of micropumps is only suitable for pumping finite volumes of fluid. Pneumatically driven aperiodic displacement pumps have been used in miniaturized drug delivery systems and the recharging of pressure source is required after long-term use. Other methods to drive aperiodic displacement micropumps include thermal or electrochemical phase change, electrical control of interfacial tension (also called electrowetting), thermocapillary effects, osmosis, and interactions of local electric fields.

\section{Rotary displacement micropumps}

Of a small number of rotary displacement micropumps reported, most were micro gear pumps. There are several main challenges that limit the development of such micropumps: 1) It is achievable to manufacture micro gear structures but very difficult to minimize the gaps between the gears and the housing, through which backflow can occur. 2) The operation backpressure of gear pumps generally scales with the inverse of viscosity so the best-suited working fluids for these pumps are moderately high viscosity liquids. 3) External motors used to drive gear pumps limit the possibility of miniaturization while other driving methods are still unsatisfactory. 


\subsubsection{Current developments of valveless piezoelectric micropump}

Valveless piezoelectric micropump belongs to the category of reciprocating displacement micropumps. Piezoelectric bending actuator is used to drive the pump diaphragm oscillating periodically and inlet/outlet channels with suitable geometries rectify the fluid flow. This type of pump can provide a broad range of flow rates and pressure head with the minimum risk of valve wear, fatigue and clogging. Both experimental and theoretical research has been conducted on this subject since the first piezoelectric valve-less micropump was introduced in 1993 [23].

It is Swedish researchers, E. Stemme and G. Stemme who developed one of the earliest piezoelectric valve-less micropumps in 1993 [23]. In this micropump, conical nozzle/diffuser elements with small opening angles $\left(<20^{\circ}\right)$ were used to rectify the flow. The diameter of the pump chamber was $19 \mathrm{~mm}$ and the pumping frequency was of the order of $100 \mathrm{~Hz}$. Using water as working fluid, the maximum flow rate was $16 \mathrm{ml} / \mathrm{min}$ and the maximum pressure head was 2 $\mathrm{mH}_{2} \mathrm{O}$. Anders Olsson et al. continued their research on this micropump. They presented a new design with two pump chambers connected in parallel in 1995 [27] and later fabricated it on silicon [28, 29]. The performance of different nozzle/diffuser elements was also investigated [30]. A lumped-mass model for this pump was established using MATLAB [31] and a CFD model using ANSYS/Flotran was applied to analyze the flow directing ability of nozzle/diffuser elements [32]. All of these works were also summarized in [33]. 
As mentioned above, Torsten Gerlach et al. from Germany used pyramidic nozzle/diffuser elements with an opening angle of $70.5^{\circ}$ in their micropumps [24, 25]. The pump diaphragm consisted of a Pyrex glass foil with a thickness of $120 \mu m$ and a PZT disk with a thickness of $200 \mu m$. The pump housing and the nozzle/diffuser elements were fabricated on a silicon wafer. A prototype with $7 \times 7 \mathrm{~mm}$ pump diaphragm reached flow rates of $400 \mu \mathrm{l} / \mathrm{min}$ for water at an excitation frequency between $1 \mathrm{kHz}$ and $10 \mathrm{kHz}$.

The valvular circuit developed by Fred K. Forster et al. from US also showed flow directing ability in micropumps [26]. A linearized dynamic system model was established to help improve the original design and dramatic improvements were obtained [34]. They also utilized the finite element method to find the optimal geometric and material parameters for the circular piezoelectric micropump actuator [35].

The earlier micropump design presented by M. Koch et al. from UK utilized passive cantilever valves to control the fluid flow [36, 37]. The pump actuator was fabricated by screen-printing a piezoelectric layer onto a silicon membrane ( $8 \mathrm{~mm}$ x $4 \mathrm{~mm} \times 70 \mu \mathrm{m})$. Using ethanol as working fluid, the maximum flow rate was $120 \mu \mathrm{l} / \mathrm{min}$ and the maximum pressure head was $2 \mathrm{kPa}$ at an excitation voltage of $600 \mathrm{~V}$ peak to peak and an excitation frequency of $200 \mathrm{~Hz}$. Later the improved design with the same nozzle/diffuser elements as used in Torsten Gerlach's [24,25] were fabricated and tested. With ethanol as test liquid, flow rates up to $155 \mu \mathrm{l} / \mathrm{min}$ and a maximum pressure head of $1 \mathrm{kPa}$ were achieved at the same driving voltage of $600 \mathrm{~V}$ peak to peak [38].

Other than micromachined technology and MEMS technology, Nam-Trung Nguyen et al from Singapore have also used printed circuit board technique to fabricate piezoelectric valveless micropump [39]. Nozzle/diffuse elements with an opening angle of $4.29^{\circ}$ were used. The pump 
diaphragm consisted two layers: the brass disk with a diameter of $15 \mathrm{~mm}$ and a thickness of 95 $\mu m$, the piezoelectric layer with a diameter of $12 \mathrm{~mm}$ and a thickness of $175 \mu \mathrm{m}$. Peristaltic micropump has also been investigated. The maximum flow rate was $3 \mathrm{ml} / \mathrm{min}$ at low driving frequencies up to $100 \mathrm{~Hz}$. Numerical simulations of such pumps using ANSYS/Flotran and CFD/ACE were given in [40]. They have also tried to make micropumps on polymeric materials [41].

The research introduced above focused more on the fabrication and test of valveless piezoelectric micropumps. Analytical modeling and numerical modeling are often used to optimize the design parameters and operation condition. Amos Ullmann from Israel established some simple analytical models to analyze the performance of single and double chamber micropumps [42]. Later he proposed an improved dynamic model so that the dynamic effects of the micropump especially at the inlet and outlet lines could be taken into account [43]. The more detailed model to study the influence of the fluid viscosity to fluid flow through the micropump channels was presented by Oana Tatiana Nedelcu and Victor Moagar-Poladian [44]. Due to the complexity of their model, only the numerical results were obtained and it was found that the pump efficiency was higher for smaller frequencies. They also conducted an ANSYS analysis for pump membrane deflection in various conditions. The analytical solutions for the deflection of circular PZT pumps actuator caused only by driving voltage have recently been reported $[45,46]$. The analytical results they obtained showed good agreement with experimental data and numerical results. All these models can provide very useful insights about the design and operation of micropumps. However, due to the complicated coupling effects between the vibration of pump actuator and the flow of working liquid, there are still some discrepancies between these models and the actual micropumps with liquid loading. 
Besides academic efforts, some companies have been working on this valveless piezoelectric micropump as well. Fig. 25 shows the photo of some micropump products developed by PAR Technologies, Inc. These micropumps are made of plastic or metal and the excitation voltage is up to $400 \mathrm{~V}$ peak to peak from 3-12 V DC input. Table 2 shows the technical data for the smallest model, LPD-30S.

Table 2 Technical data of pump model LPD-30S

\begin{tabular}{|c|c|}
\hline Model No. & LPD-30S \\
\hline Flow Rate & Up to $30 \mathrm{ml} / \mathrm{min}$ \\
\hline Pumping Frequency & $130 \mathrm{~Hz}$ \\
\hline Pressure Head & $0-1 \mathrm{psi}$ \\
\hline Input Voltage & $2.5-12 \mathrm{VDC}$ \\
\hline Current Draw & $10-130 \mathrm{~mA}$ \\
\hline Size & $0.63 \times 0.63 \times 0.21$ inch \\
\hline
\end{tabular}

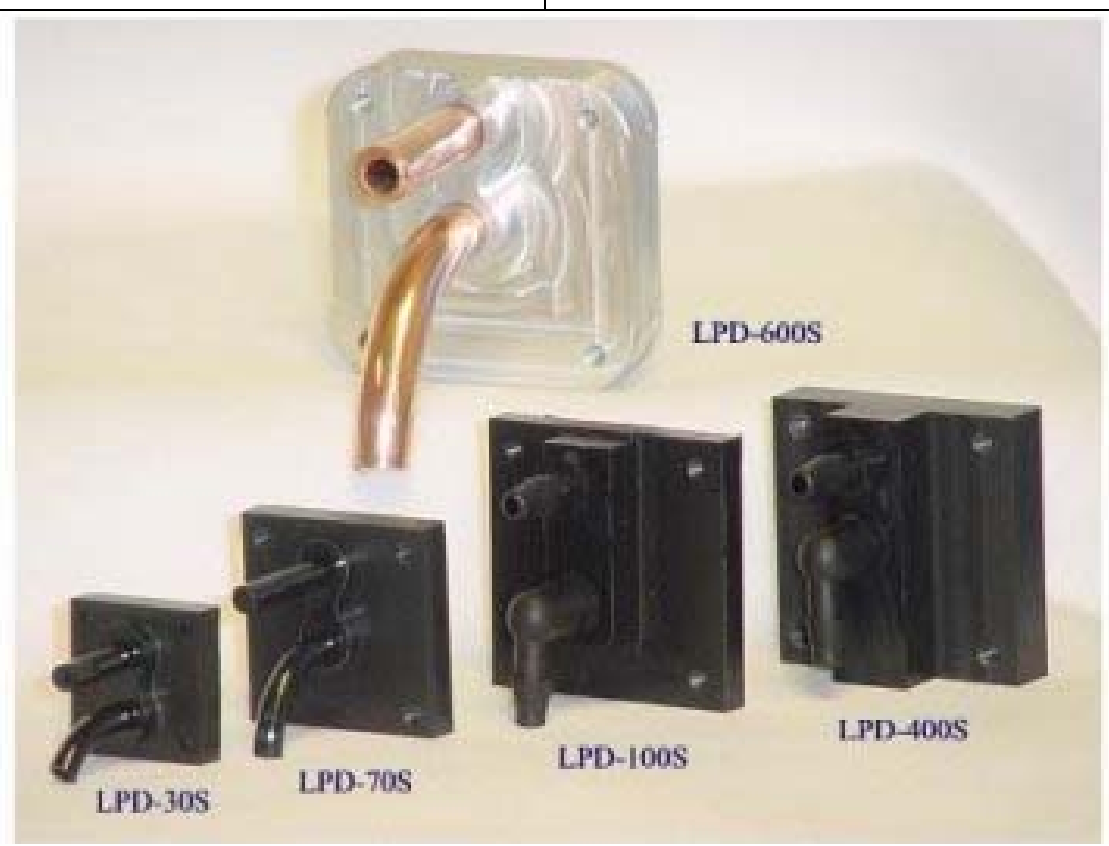

Figure 25 Photo of the micropump prototypes developed by PAR Technologies, Inc. 


\subsection{SUMMARY}

An extensive review of both fuel cell and micropump has been presented including the history, working principle, types, and current research status. They are very useful technologies and have already been used in many applications. To improve their performance and fit into the specific applications, a lot of challenges still have to be solved.

As mentioned earlier, DMFC is a promising candidate for portable power applications and many prototypes have already been developed. However, the performance of such system is still unsatisfactory due to many factors such as slower electrochemical reaction, methanol crossover, fuel delivery, oxidant supply, water management, thermal management and etc. Of them, fuel delivery is the focus of this research and it is expected that micropump technology can be applied to solve this problem effectively. 


\subsection{RESEARCH OBJECTIVE}

The objective of this research is to develop a simple, efficient and compact power system for portable electronics by combining fuel cell technology with micropump technology. As mentioned in previous chapters, DMFC is a preferable choice for commercial applications because methanol solution is much easier to deal with as fuel than hydrogen. To improve its more sluggish performance comparing to PEFC, an appropriate fuel delivery system will play an important role. Therefore, a compact DMFC power system driving by valveless piezoelectric micropump is presented here and it is expected that the fuel delivery problem will be solved well in this system.

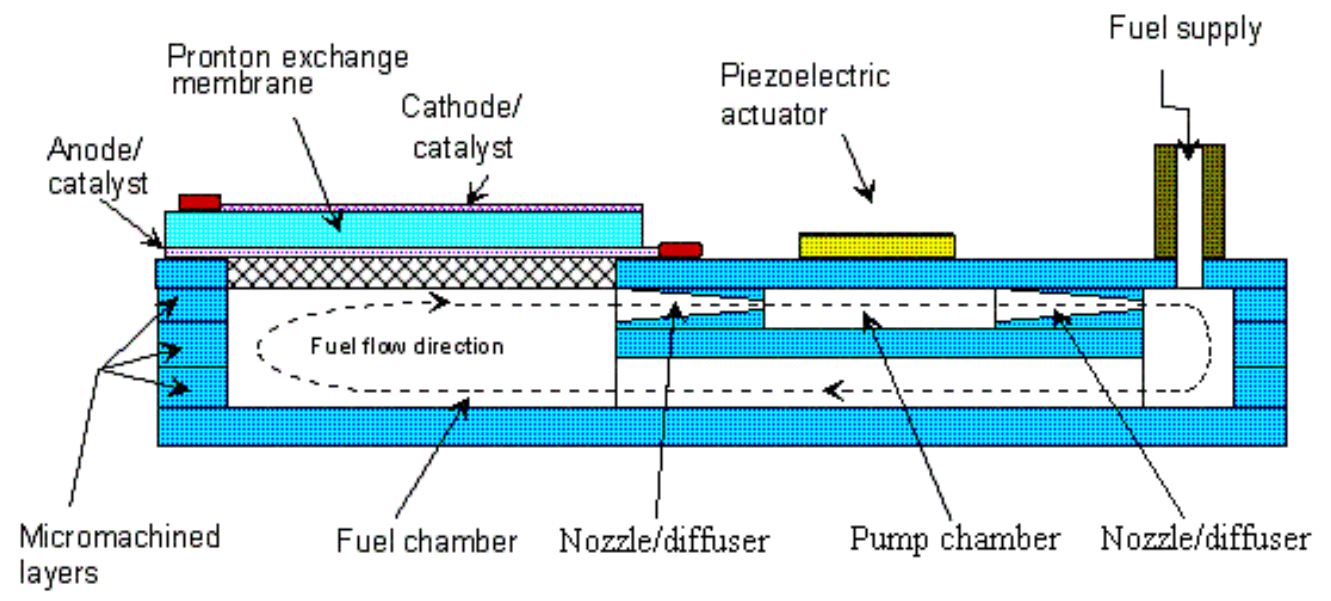

Figure 26 Schematic of the miniaturized direct methanol fuel cell (DMFC) power system using valveless piezoelectric micropump for fuel delivery

The cross-section of the DMFC power system with valveless piezoelectric micropump is shown schematically in Fig. 26. Note that the figure is not drawn in the actual scale. This system mainly consists of the following parts: fuel cell membrane electrode assembly (MEA), fuel chamber, 
nozzle/diffuser, micropump and pump chamber, and fuel supply manifold. All these parts are fabricated in a multi-layer structure to obtain a compact system. The fuel cell MEA is made of a Nafion ${ }^{\circledR} 117$ membrane layer sandwiched by two electrode layers with catalysts deposited on them. And the micropump is fabricated by bonding a thin piezoelectric disk on a metal diaphragm. When applying an alternating voltage to the piezoelectric disk, the diaphragm is actuated to produce bending deformation that causes the volume change of pump chamber. By selecting appropriate shape and dimension of the nozzle/diffuser between pump chamber and fuel chamber, the fuel can circulate in the desired direction. And the fuel supply from the right chamber can compensate the fuel consumption.

Before the performance of this whole system can be evaluated, it is necessary to design a micropump that can fit the requirements of the system. First, certain flow rate has to be obtained by this micropump so that enough fuel can be supplied for the electrochemical reaction and generated carbon dioxide can be removed from the reaction site by the flow. Second, since the power that is used to drive the micropump comes from the fuel cell itself, it is very important to keep this power consumption as small as possible. To calculate the flow rate and the power consumption, a theoretical model of the micropump is established in the next chapter. 


\subsection{THEORETICAL ANALYSIS OF MICROPUMP}

The most important part of a micropump is the actuator, which in this case is actually a piezoelectric bending actuator made of three layers: PZT layer, bonding layer and passive plate. When applying an alternating electrical field across the PZT layer, it will generate a reciprocating deflection in the direction vertical to the surface of the actuator. This deflection is then transferred to the pumping effect that drives the fluid inside the pump chamber flowing through the inlet/outlet. Therefore the first thing need to do in the micropump modeling is to calculate this deflection. In this chapter, two types of bending actuators have been investigated: one is the disk-type bending actuator, and the other is the ring-type bending actuator. Both can be used to drive the micropump.

\subsection{DEFLECTION OF DISK-TYPE BENDING ACTUATOR}

\subsubsection{Models developed by previous researchers}

One of the earliest models to estimate the deflection of the disk-type bending actuator was presented in 1973 [47]. The actuator investigated in this paper was used in ink jet printers. The schematic of this model is shown in Fig. 27. In this model, the bonding layer is ignored and the radius of the PZT disk is as same as that of the passive plate. The edges of both PZT disk and the passive plate are fixed. 


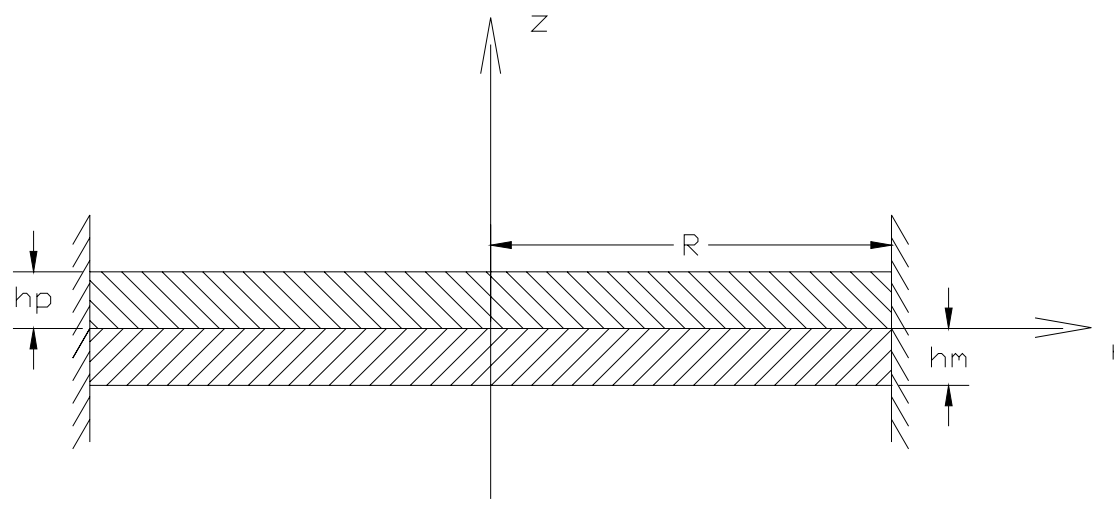

Figure 27 Schematic of the pump actuator model in [47]

Under the assumption of pure bending, the expression for the deflection of such a structure is given by Eq. (3.1-3.2) from [47],

$$
\begin{aligned}
& w(r) \approx \frac{R^{2}-r^{2}}{h_{p}^{2}\left[3\left(1-\alpha^{2} \gamma\right)^{2}-4(1+\alpha \gamma)\left(1+\alpha^{3} \gamma\right)\right]} \\
& \cdot\left[\frac{3 s_{11}^{E}\left(1-v_{p}^{2}\right)(1+\alpha \gamma)}{16 h_{p}} \cdot\left(r^{2}-\frac{5+v_{p}}{1+v_{p}} R^{2}\right) p_{p l}+3 d_{31}(1+\alpha) \alpha \gamma U\right]
\end{aligned}
$$

Here,

$$
\begin{aligned}
& \alpha=h_{m} / h_{p} \\
& \gamma=s_{11}^{E} \times E_{m} \times\left(1-v_{p}^{2}\right) /\left(1-v_{m}^{2}\right)
\end{aligned}
$$

$h_{p}, h_{m}$ are the thickness of the PZT disk and the passive plate; $v_{p}, v_{m}$ are the Poisson's ratio of the PZT disk and the passive plate; $E_{m}$ is the Young's modulus of the passive plate; $s_{11}^{E}$ is the elastic compliance of the PZT disk at constant electric field; $d_{31}$ is the piezoelectric charge coefficient and $R$ is the radius of the whole circular structure. As shown in Eq. (3.1), the total deflection consists of two parts: one is proportional to the pressure difference exerted on the whole structure, $p_{p l}$; the other is proportional to the applied voltage across the PZT disk, $U$. 
The volume change of the pump chamber induced by this deflection can be calculated by Eq. (3.3-3.5) from [47],

$$
\begin{gathered}
\Delta V=2 \pi \int_{0}^{R} w(r) r d r \\
\Delta V \approx-\frac{\pi s_{11}^{E}\left(1-v_{p}\right)\left(7+v_{p}\right)(1+\alpha \gamma) R^{6}}{16 h_{p}^{3}\left[3\left(1-\alpha^{2} \gamma\right)^{2}-4(1+\alpha \gamma)\left(1+\alpha^{3} \gamma\right)\right]} \\
\cdot\left[p_{p l}-\frac{24 h_{p} d_{31}(1+\alpha) \alpha \gamma}{s_{11}^{E} R^{2}\left(1-v_{p}\right)\left(7+v_{p}\right)(1+\alpha \gamma)} U\right] \\
=k_{1} p_{p l}+k_{2} U
\end{gathered}
$$

Here,

$$
\begin{aligned}
& k_{1}=-\frac{\pi s_{11}^{E}\left(1-v_{p}\right)\left(7+v_{p}\right)(1+\alpha \gamma) R^{6}}{16 h_{p}^{3}\left[3\left(1-\alpha^{2} \gamma\right)^{2}-4(1+\alpha \gamma)\left(1+\alpha^{3} \gamma\right)\right]} \\
& k_{2}=\frac{3 \pi d_{31}(1+\alpha) \alpha \gamma R^{4}}{2 h_{p}^{2}\left[3\left(1-\alpha^{2} \gamma\right)^{2}-4(1+\alpha \gamma)\left(1+\alpha^{3} \gamma\right)\right]}
\end{aligned}
$$

Similar to the deflection, the volume change also consists of two parts: one is proportional to the pressure difference, and the other is proportional to the applied voltage. If an alternating voltage is applied to the piezoelectric disk and the driving frequency is far less than the resonant frequency of the system, $U$ can then be substituted by $U_{m} \sin (\omega t)$.

In most of the applications for micropumps, the PZT disk is smaller than the passive plate and therefore the edge of the PZT disk is often considered to be free. A model that is more accurate for such cases has recently been developed [45]. Fig. 28 shows the basic structure of the valveless piezoelectric micropump studied in this literature. The schematic of the actuator part is illustrated in Fig. 29. As shown in the figure, the PZT disk is adhered to the passive plate by a thin layer of conductive epoxy. 


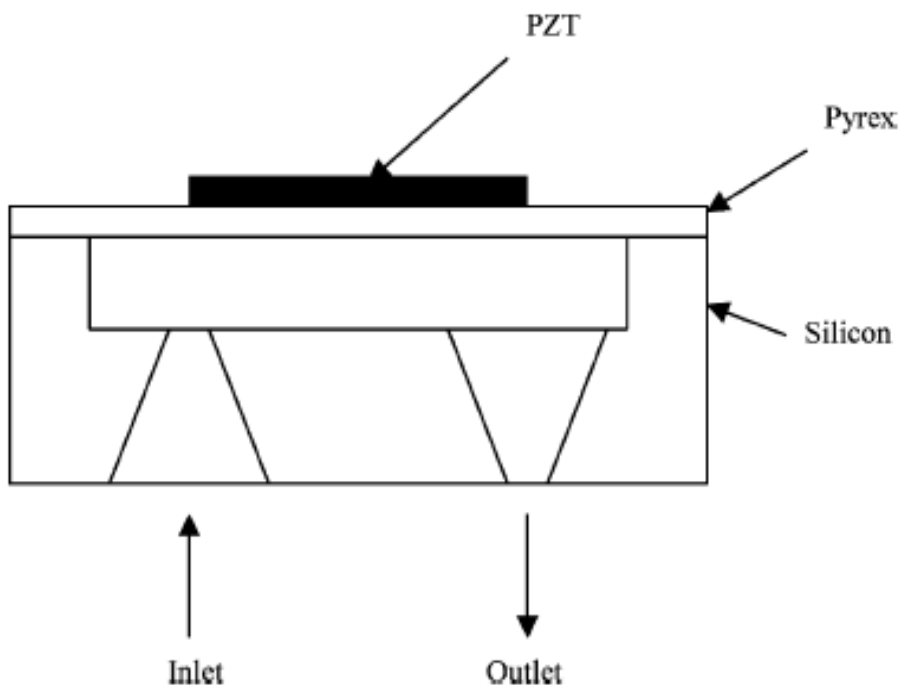

Figure 28 Schematic of the micropump design in [45]

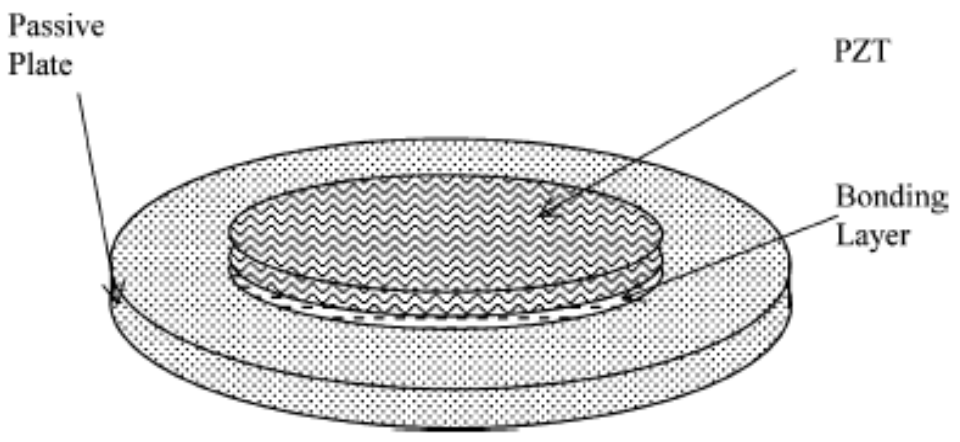

Figure 29 Schematic of the PZT disk-type bending actuator

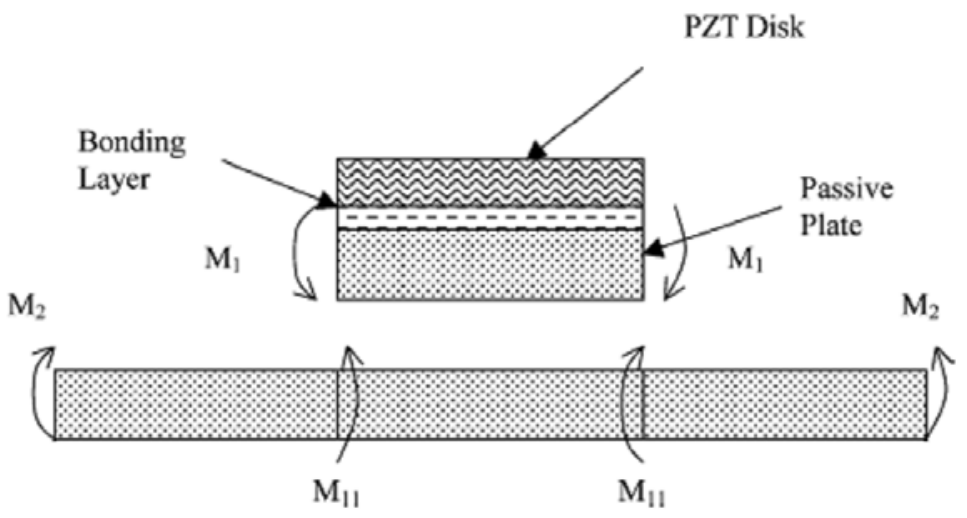

Figure 30 Schematic of the pump actuator model for analysis 


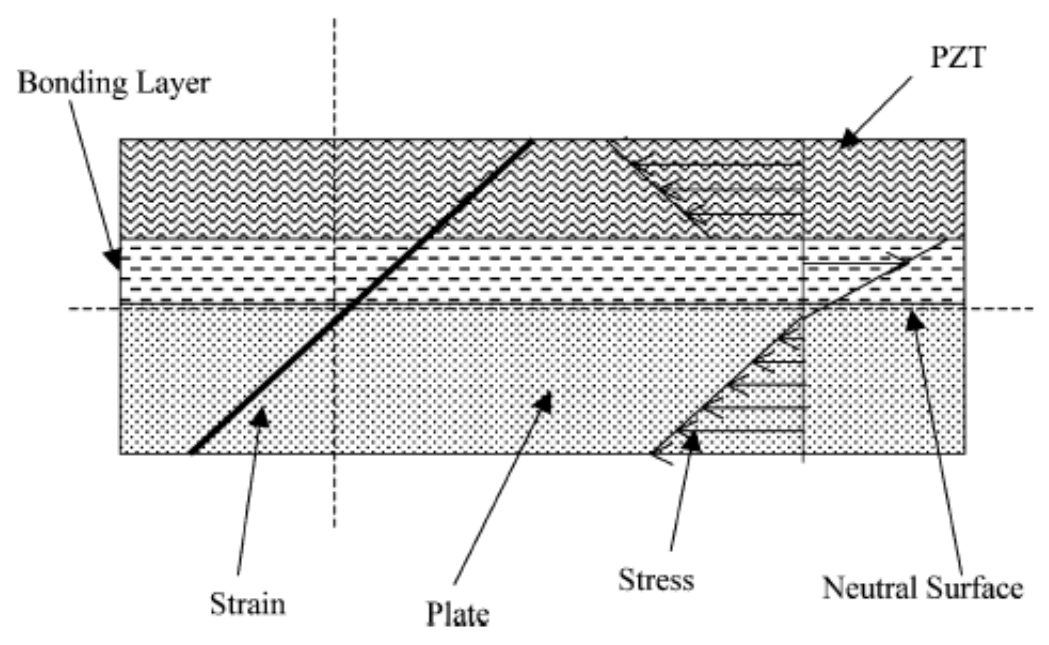

Figure 31 Strain and stress distribution of the three-layer structure

To model this bending actuator, the whole structure is divided into two parts: one is the threelayer structure including PZT disk, bonding layer and passive plate, and the other is the rest part of passive plate that has the shape of a ring (Shown in Fig. 30). Since the thickness of the threelayer structure is very small, it can be assumed that the strain distribution across the thickness direction is linear. Also assume that there exists a neutral surface that has no transverse or radial strain. These assumptions are shown in Fig. 31. The detailed derivation of this model is given below.

Based on the work done in [48], the location of the neutral surface is

$$
h=\frac{1}{2} \frac{\left.\left.E_{p} h_{p}^{2} /\left(1-\gamma_{p}^{2}\right)+E_{b} \mid\left(h_{p}+h_{b}\right)^{2}-h_{p}^{2}\right\rfloor /\left(1-\gamma_{b}^{2}\right)+E_{p z t} \mid\left(h_{p}+h_{b}+h_{p z t}\right)^{2}-\left(h_{p}+h_{b}\right)^{2}\right\rfloor /\left(1-\gamma_{p z t}^{2}\right)}{E_{p} h_{p} /\left(1-\gamma_{p}^{2}\right)+E_{b} h_{b} /\left(1-\gamma_{b}^{2}\right)+E_{p z t} h_{p z t} /\left(1-\gamma_{p z t}^{2}\right)}(3.6)
$$

Here, $E_{p}, E_{b}$, and $E_{p z t}$ represent the Young's modulus of the passive plate, the bonding layer and the PZT disk respectively. $\gamma_{p}, \gamma_{b}$, and $\gamma_{p z t}$ represent the Poisson's ratio of the passive plate, the bonding layer and the PZT disk respectively. $h_{p}, h_{b}$, and $h_{p z t}$ represent the thickness of the passive plate, the bonding layer and the PZT disk respectively. 
For the bonding layer and the passive plate, stress $\sigma$ and strain $\varepsilon$ satisfy that

$$
\sigma_{x}=\frac{E_{x}}{1-\gamma_{x}^{2}} \varepsilon_{x}
$$

The subscript $x$ can be either $p$ or $b$.

Due to the linear strain assumption, we have

$$
\varepsilon(z)=z k
$$

We also have

$$
\frac{\sigma_{p}}{\sigma_{i}}=\frac{z}{h_{p}-h} \Rightarrow \sigma_{p}=\frac{\sigma_{i}}{h_{p}-h} z
$$

where $z$ is the deflection from the neutral surface, $\sigma_{i}$ is the stress at the interface between the bonding layer and the passive plate, and $h$ is the distance between the neutral surface and the bottom surface of the passive plate.

Combining these three equations, the slope of the strain $k$ can be obtained

$$
k=\frac{1-\gamma_{p}^{2}}{E_{p}} \frac{\sigma_{i}}{h_{p}-h}
$$

Therefore, the stress distribution in the bonding layer can be expressed as

$$
\sigma_{b}=\frac{E_{b}}{1-\gamma_{b}^{2}} k z=\frac{1-\gamma_{p}^{2}}{1-\gamma_{b}^{2}} \frac{E_{b}}{E_{p}} \frac{\sigma_{i}}{h_{p}-h} z
$$

And using the linear piezoelectric constitutive equations, the stress distribution inside the PZT layer is

$$
\sigma_{p z t}=\frac{E_{p z t}}{1-\gamma_{p z t}^{2}}\left(k z-\frac{U}{h_{p z t}} d_{31}\right)=\frac{1-\gamma_{p}^{2}}{1-\gamma_{p z t}^{2}} \frac{E_{p z t}}{E_{p}} \frac{\sigma_{i}}{h_{p}-h} z-\frac{E_{p z t}}{1-\gamma_{p z t}^{2}} \frac{U}{h_{p z t}} d_{31}
$$

Here, $U$ is the applied voltage and $d_{31}$ is the piezoelectric charge coefficient. 
From the momentum balance of the three-layer structure, we have

$$
\int_{-h}^{h_{p}-h} \sigma_{p} z d z+\int_{h_{p}-h}^{h_{p}-h+h_{b}} \sigma_{b} z d z+\int_{h_{p}-h+h_{b}}^{h_{p}-h+h_{b}+h_{p z t}} \sigma_{p z t} z d z=0
$$

Substituting Eqs. (3.9), (3.11) and (3.12) into the equation above, we obtain that

$$
\sigma_{i}=\frac{3 \eta U d_{31}\left(2 h^{\prime}+2 h_{b}+h_{p z t}\right) h^{\prime}}{2\left\{h^{\prime 3}+h^{3}+\alpha\left[\left(h^{\prime}+h_{b}\right)^{3}-h^{\prime 3}\right]+\beta\left[\left(h^{\prime}+h_{b}+h_{p z t}\right)^{3}-\left(h^{\prime}+h_{b}\right)^{3}\right]\right\}}
$$

Here,

$$
h^{\prime}=h_{p}-h, \alpha=\frac{1-\gamma_{p}^{2}}{1-\gamma_{b}^{2}} \frac{E_{b}}{E_{p}}, \beta=\frac{1-\gamma_{p}^{2}}{1-\gamma_{p z t}^{2}} \frac{E_{p z t}}{E_{p}}, \eta=\frac{E_{p z t}}{1-\gamma_{p z t}^{2}}
$$

The moment $M_{1}$ is given by

$$
M_{1}=\int_{-h}^{h^{\prime}} \sigma_{p} z d z=\frac{\eta U d_{31}\left(2 h^{\prime}+2 h_{b}+h_{p z t}\right)\left(h^{\prime 3}+h^{3}\right)}{2\left[h^{3}+(1-\alpha) h^{\prime 3}+(\alpha-\beta)\left(h^{\prime}+h_{b}\right)^{3}+\beta\left(h^{\prime}+h_{b}+h_{p z t}\right)^{3}\right]}
$$

Using the equations derived from [49], the effective Young's modulus and Poisson's ratio for the three-layer structure can be calculated. First, for the PZT and the bonding layer, the effective properties are:

$$
\begin{gathered}
E^{\prime}=C_{1} E_{p z t}+C_{2} E_{b}+\frac{C_{1} C_{2} E_{p z t} E_{b}\left(\gamma_{p z t}-\gamma_{b}\right)^{2}}{C_{1} E_{p z t}\left(1-\gamma_{b}^{2}\right)+C_{2} E_{b}\left(1-\gamma_{p z t}^{2}\right)} \\
\gamma^{\prime}=\frac{C_{1} \gamma_{p z t} E_{p z t}\left(1-\gamma_{b}^{2}\right)+C_{2} \gamma_{b} E_{b}\left(1-\gamma_{p z t}^{2}\right)}{C_{1} E_{p z t}\left(1-\gamma_{b}^{2}\right)+C_{2} E_{b}\left(1-\gamma_{p z t}^{2}\right)}
\end{gathered}
$$

Here,

$$
h^{\prime \prime}=h_{p z t}+h_{b}, C_{1}=h_{p z t} / h^{\prime \prime}, C_{2}=h_{b} / h^{\prime \prime}
$$

Now, as for the whole structure, the effective properties can be derived: 


$$
\begin{gathered}
E_{c}=C_{1}^{\prime} E_{p}+C_{2}^{\prime} E^{\prime}+\frac{C_{1}^{\prime} C_{2}^{\prime} E_{p} E^{\prime}\left(\gamma_{p}-\gamma^{\prime}\right)^{2}}{C_{1}^{\prime} E_{p}\left(1-\gamma^{\prime 2}\right)+C_{2}^{\prime} E^{\prime}\left(1-\gamma_{p}^{2}\right)} \\
\gamma_{c}=\frac{C_{1}^{\prime} \gamma_{p} E_{p}\left(1-\gamma^{\prime 2}\right)+C_{2}^{\prime} \gamma^{\prime} E^{\prime}\left(1-\gamma_{p}^{2}\right)}{C_{1}^{\prime} E_{p}\left(1-\gamma^{\prime 2}\right)+C_{2}^{\prime} E^{\prime}\left(1-\gamma_{p}^{2}\right)}
\end{gathered}
$$

Here,

$$
h_{\text {all }}=h_{p z t}+h_{b}+h_{p}, C_{1}^{\prime}=h_{p} / h_{\text {all }}, C_{2}^{\prime}=h^{\prime \prime} / h_{\text {all }}
$$

As mentioned before, the whole pump actuator is divided into two parts and therefore the deflections of them can be calculated separately. For the rest part of the passive plate, its deflection $w_{2}$ is obtained using the equations given by [50],

$$
w_{2}(r)=\frac{M_{11} a^{2}}{2 D_{p}\left[\left(1-\gamma_{p}\right) b^{2}+\left(1+\gamma_{p}\right) a^{2}\right]} \times\left(-r^{2}+2 b^{2} \log \frac{r}{b}+b^{2}\right)
$$

For the three-layer structure, its deflection $w_{1}$ also follows [50],

$$
w_{1}(r)=\frac{M_{11} a^{2}}{2 D_{p}\left[\left(1-\gamma_{p}\right) b^{2}+\left(1+\gamma_{p}\right) a^{2}\right]} \times\left(-a^{2}+2 b^{2} \log \frac{a}{b}+a^{2}\right)+\frac{M_{1}}{2 D_{c}\left(1+\gamma_{c}\right)}\left(a^{2}-r^{2}\right)
$$

Here, $a$ represents the radius of the PZT disk and $b$ represents the radius of the whole passive plate. The flexural modulus for the rest part of the passive plate and the three-layer structure are given by

$$
D_{p}=\frac{E_{p} h_{p}^{3}}{12\left(1-\gamma_{p}^{2}\right)}, \quad D_{c}=\frac{E_{c} h_{a l l}^{3}}{12\left(1-\gamma_{c}^{2}\right)}
$$

To satisfy the continuity condition,

$$
\left.\frac{d w_{1}(r)}{d r}\right|_{r=a}=\left.\frac{d w_{2}(r)}{d r}\right|_{r=a}
$$

Substituting Eqs. (3.23) and (3.24) into (3.26), the moment $M_{11}$ is found to be 


$$
M_{11}=-\frac{M_{1} D_{p}\left[\left(1-\gamma_{p}\right) b^{2}+\left(1+\gamma_{p}\right) a^{2}\right]}{D_{c}\left(1+\gamma_{c}\right)\left(b^{2}-a^{2}\right)}
$$

Combining Eqs. (3.23), (3.24), (3.16) and (3.27), the deflection distribution $w(r)$ of the whole pump actuator can be fully determined. After rearrangements, the equations of the whole deflection can be rewritten as,

$$
\begin{gathered}
w_{1}(r)=\frac{M_{1}\left[\left(b^{2}-a^{2}\right)\left(a^{2}-r^{2}\right)+a^{2}\left(a^{2}-2 b^{2} \log \frac{a}{b}-b^{2}\right)\right]}{2 D_{c}\left(1+\gamma_{c}\right)\left(b^{2}-a^{2}\right)}, \quad(0 \leq r \leq a) . \\
w_{2}(r)=\frac{M_{1} a^{2}\left(r^{2}-2 b^{2} \log \frac{r}{b}-b^{2}\right)}{2 D_{c}\left(1+\gamma_{c}\right)\left(b^{2}-a^{2}\right)}, \quad(a \leq r \leq b) .
\end{gathered}
$$

The volume change of the pump chamber induced by this deflection can be calculated using Eq. (3.3). Note that in this case,

$$
w(r)=\left\{\begin{array}{ll}
w_{1}(r), & 0 \leq r<a ; \\
w_{2}(r), & a \leq r \leq b .
\end{array} \text { and } R=b .\right.
$$

It can be shown that the volume change of the pump chamber $\Delta V$ is ultimately proportional to the applied voltage $U$ and can be written as

$$
\Delta V=k_{e f f} U
$$

The proportional coefficient $k_{\text {eff }}$ only depends on the dimensions and the material properties of the pump actuator. The same conclusion has been obtained in the model mentioned previously. Also $U$ can be substituted by $U_{m} \sin (\omega t)$ under certain circumstances. The deflection caused by pressure difference is not considered in this model. 
Another similar model [46] has been developed with some major differences. The schematic of this model is shown in Fig. 32. The whole structure is also divided into two parts. This step is just the same as in the previous model.

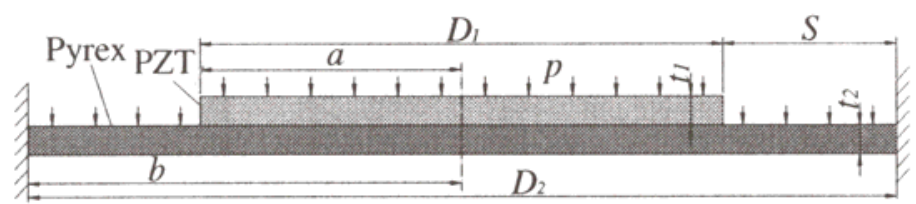

(a)

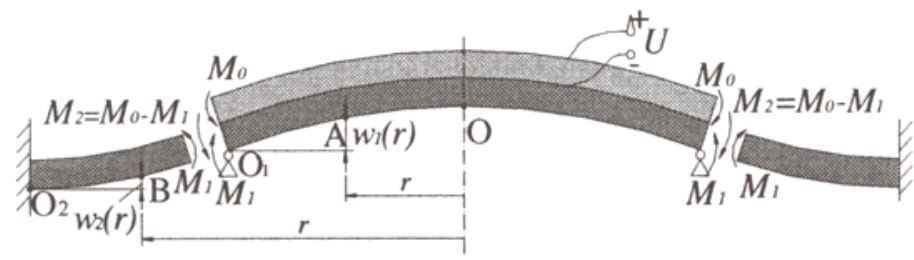

(b)

Figure 32 Schematic of the pump actuator model in [46]

In this model, the bonding layer is neglected so the central part is only a two-layer structure. For this two-layer part, simply supported at $r=a$, the deflection at point $A$ relative to the supporting point $O_{1}$ is

$$
w_{1}^{\prime}(r)=\frac{M_{2}}{2 D_{e}\left(1+v_{e}\right)}\left(a^{2}-r^{2}\right), \quad(0 \leq r \leq a) .
$$

Here $D_{e}$ and $v_{e}$ are the equivalent flexural modulus and the equivalent Poisson's ration of the two-layer structure, respectively. $M_{2}$ is the intermediate moment applied at the outer edge of the two-layer structure and $a$ is the radius of the PZT disk.

The outer part is actually a ring with outer edge fixed supported at $r=b$, inner edge free. Its deflection at point $B$ relative to the supporting point $O_{2}$ is

$$
w_{2}(r)=\frac{M_{1} a^{2}\left(r^{2}-2 b^{2} \log \frac{r}{b}-b^{2}\right)}{2 D_{s 2}\left[\left(1+v_{2}\right) a^{2}+\left(1-v_{2}\right) b^{2}\right]}, \quad(a \leq r \leq b) .
$$


Here $D_{s 2}$ and $v_{2}$ are the flexural modulus and the Poisson's ration of the Pyrex, respectively. $M_{1}$ is the intermediate moment applied at the inner edge of the ring structure and $b$ is the radius of the whole Pyrex plate.

The relationship between the two intermediate moments can be expressed as

$$
M_{2}=M_{0}-M_{1}
$$

From [51], the moment caused by actuation of the PZT is

$$
M_{0}=D_{e} \frac{-d_{31} U / t_{1}}{\frac{h}{2}+\frac{2}{h}\left(\frac{1}{E_{11} t_{1}}+\frac{1}{E_{2} t_{2}}\right)\left(D_{s 1}+D_{s 2}\right)}
$$

Here $U$ is the voltage applied on the PZT disk and $h$ is the total thickness of the pump actuator. $t_{1}, t_{2}$ represent the thickness of the PZT disk and the passive plate, respectively. $D_{s 1}, E_{11}$ and $d_{31}$ are the flexural modulus, Young's modulus and the piezoelectric charge coefficient of the PZT, respectively. $E_{2}$ is the Young's modulus of the passive plate.

Therefore the maximum deflection of the pump actuator at point $O$ can be calculated by

$$
w_{\max }=w_{1}^{\prime}(0)+w_{2}(a)
$$

The deflection of the whole structure relative to the supporting point $O_{2}$ can be summarized as

$$
\begin{gathered}
w_{1}(r)=w_{1}^{\prime}(r)+w_{2}(a)=\frac{M_{2}}{2 D_{e}\left(1+v_{e}\right)}\left(a^{2}-r^{2}\right)+\frac{M_{1} a^{2}\left(a^{2}-2 b^{2} \log \frac{a}{b}-b^{2}\right)}{2 D_{s 2}\left[\left(1+v_{2}\right) a^{2}+\left(1-v_{2}\right) b^{2}\right]}, \quad(0 \leq r \leq a) . \\
w_{2}(r)=\frac{M_{1} a^{2}\left(r^{2}-2 b^{2} \log \frac{r}{b}-b^{2}\right)}{2 D_{s 2}\left[\left(1+v_{2}\right) a^{2}+\left(1-v_{2}\right) b^{2}\right]}, \quad(a \leq r \leq b) .
\end{gathered}
$$

Also by satisfying the continuity condition Eq. (3.26), it is found that,

$$
M_{1}=M_{0} \frac{D_{s 2}\left[\left(1+v_{2}\right) a^{2}+\left(1-v_{2}\right) b^{2}\right]}{D_{s 2}\left[\left(1+v_{2}\right) a^{2}+\left(1-v_{2}\right) b^{2}\right]+D_{e}\left(1+v_{e}\right)\left(b^{2}-a^{2}\right)}
$$


Therefore the deflection of the whole structure can be rewritten as,

$$
\begin{aligned}
& w_{1}(r)=\frac{M_{0}\left[\left(b^{2}-a^{2}\right)\left(a^{2}-r^{2}\right)+a^{2}\left(a^{2}-2 b^{2} \log \frac{a}{b}-b^{2}\right)\right]}{2\left\{D_{s 2}\left[\left(1+v_{2}\right) a^{2}+\left(1-v_{2}\right) b^{2}\right]+D_{e}\left(1+v_{e}\right)\left(b^{2}-a^{2}\right)\right\}}, \quad(0 \leq r \leq a) . \\
& w_{2}(r)=\frac{M_{0} a^{2}\left(r^{2}-2 b^{2} \log \frac{r}{b}-b^{2}\right)}{2\left\{D_{s 2}\left[\left(1+v_{2}\right) a^{2}+\left(1-v_{2}\right) b^{2}\right]+D_{e}\left(1+v_{e}\right)\left(b^{2}-a^{2}\right)\right\}}, \quad(a \leq r \leq b) .
\end{aligned}
$$

Same as the previous model, the volume change of the pump chamber can be determined using Eq. (3.3) and the final result can be expressed using Eq. (3.31).

There are two major differences between this model and the previous model: the modeling of the bonding layer and the calculation of the moment caused by PZT actuation. Since the influence of the bonding layer is actually very small, the latter one is more critical. Both models did not give a theoretical solution to the deflection caused by pressure but included this deflection in the numerical analysis.

\subsubsection{Modified model for disk-type bending actuator}
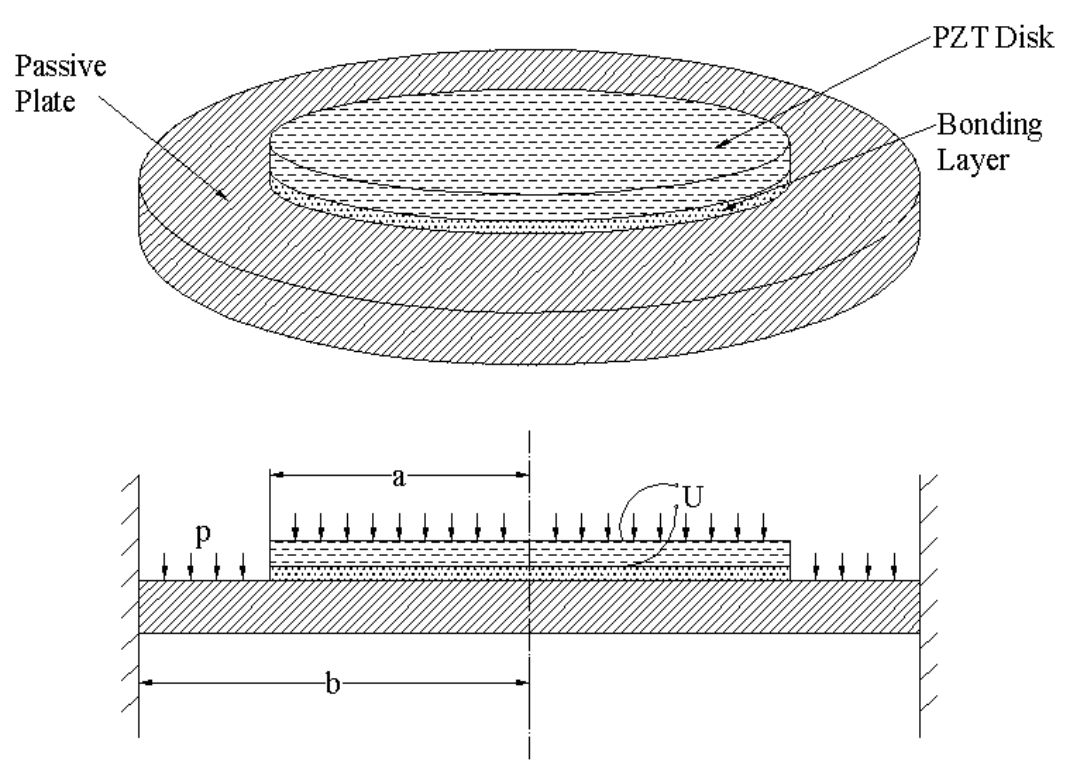
Figure 33 Schematic of the PZT disk-type bending actuator model

Based on the models introduced above, a modified theoretical model is developed to estimate the deflection of the disk-type bending actuator. The schematic of the disk-type bending actuator and its model is shown in Fig. 33.

The following assumptions are made to simplify the modeling process:

1. The whole structure is circumferentially symmetrical.

2. The thickness of each layer is much smaller than its radius and the deformation is very small in comparison with the dimension of the structure. So the whole system can be considered as thin plates with small deflection (Three assumptions of the pure bending are satisfied [50]).

3. The thickness of the bonding layer is small in comparison with the thicknesses of the PZT disk and the passive plate therefore the influence of the bonding layer on the deflection of the whole structure is neglected.

4. The bonding between the PZT disk and the passive plate is perfect.

5. The outer edge of the passive plate is fixed.

As mentioned above, the deflection of the whole structure can be caused by two factors: the applied voltage across the PZT disk, $U$, and the pressure difference exerted on the whole structure, $p$. And the evaluation of them will be carried out separately.

\section{Deflection caused by the applied voltage}

As for the deflection caused by the applied voltage, the model developed by [46] can be adopted here directly. Given that the material properties, dimensions and the applied voltage are known, this deflection is fully determined by Eqs. (31), (36) and (37). Here these equations are rewritten with the notations that will be used throughout the rest of the dissertation. 
The intermediate moment caused by the actuation of the PZT is,

$$
M_{0}=D_{e} \frac{-d_{31} U / h_{p z t}}{\frac{h}{2}+\frac{2}{h}\left(\frac{1}{E_{p z t} h_{p z t}}+\frac{1}{E_{p} h_{p}}\right)\left(D_{p z t}+D_{p}\right)}
$$

The deflection of the two-layer structure (the central part) is,

$$
w_{1}(r)=\frac{M_{0}\left[\left(b^{2}-a^{2}\right)\left(a^{2}-r^{2}\right)+a^{2}\left(a^{2}-2 b^{2} \log \frac{a}{b}-b^{2}\right)\right]}{2\left\{D_{p}\left[\left(1+v_{p}\right) a^{2}+\left(1-v_{p}\right) b^{2}\right]+D_{e}\left(1+v_{e}\right)\left(b^{2}-a^{2}\right)\right\}}, \quad(0 \leq r \leq a) .
$$

The deflection of the passive plate ring (the outer part) is,

$$
w_{2}(r)=\frac{M_{0} a^{2}\left(r^{2}-2 b^{2} \log \frac{r}{b}-b^{2}\right)}{2\left\{D_{p}\left[\left(1+v_{p}\right) a^{2}+\left(1-v_{p}\right) b^{2}\right]+D_{e}\left(1+v_{e}\right)\left(b^{2}-a^{2}\right)\right\}}, \quad(a \leq r \leq b) .
$$

Here $U$ is the voltage applied across the PZT disk; $d_{31}$ is the piezoelectric charge coefficient; $h$ is the total thickness of the PZT disk and the passive plate; $h_{p z t}, h_{p}$ are the thickness of the PZT disk and the passive plate, respectively; $E_{p z t}, E_{p}$ are the Young's modulus of the PZT disk and the passive plate, respectively; $v_{p z t}, v_{p}$ are the Poisson's ratio of PZT disk and the passive plate, respectively; $D_{p z t}, D_{p}$ are the flexural modulus of the PZT disk and the passive plate, respectively; $E_{e}, v_{e}$, and $D_{e}$ are the equivalent Young's modulus, Poisson's ratio, and flexural modulus of the two-layer structure, respectively.

The calculations of these equivalent material properties of the two-layer structure are as follows,

$$
E_{e}=C_{1} E_{p z t}+C_{2} E_{p}+\frac{C_{1} C_{2} E_{p z t} E_{p}\left(v_{p z t}-v_{p}\right)^{2}}{C_{1} E_{p z t}\left(1-v_{p}^{2}\right)+C_{2} E_{p}\left(1-v_{p z t}^{2}\right)}
$$




$$
\begin{gathered}
v_{e}=\frac{C_{1} v_{p z t} E_{p z t}\left(1-v_{p}^{2}\right)+C_{2} v_{p} E_{p}\left(1-v_{p z t}^{2}\right)}{C_{1} E_{p z t}\left(1-v_{p}^{2}\right)+C_{2} E_{p}\left(1-v_{p z t}^{2}\right)} \\
D_{e}=\frac{E_{e} h^{3}}{12\left(1-v_{e}^{2}\right)}
\end{gathered}
$$

Here,

$$
C_{1}=h_{p z t} / h, C_{2}=h_{p} / h
$$

\section{Deflection caused by the pressure}

The deflection caused by the mechanical pressure difference is also calculated respectively for the two same sections: the central part and the outer part. By using the method of superposition, the established equations from [50] can be combined together to obtain the solution for this specific case.

Under constant pressure difference $p$, the deflection of the two-layer structure is,

$$
\begin{aligned}
& w_{3}(r)=\frac{p}{64 D_{p}}\left(b^{2}-a^{2}\right)^{2}+\frac{\left(M_{1}-M_{2}\right) a^{2}\left(a^{2}-2 b^{2} \log \frac{a}{b}-b^{2}\right)}{2 D_{p}\left[\left(1-v_{p}\right) b^{2}+\left(1+v_{p}\right) a^{2}\right]} \\
& +\frac{p\left(a^{2}-r^{2}\right)}{64 D_{e}}\left(\frac{5+v_{e}}{1+v_{e}} a^{2}-r^{2}\right)+\frac{M_{2}}{2 D_{e}\left(1+v_{e}\right)}\left(a^{2}-r^{2}\right), \quad(0 \leq r \leq a) .
\end{aligned}
$$

And the deflection of the passive plate ring is,

$$
w_{4}(r)=\frac{p}{64 D_{p}}\left(b^{2}-r^{2}\right)^{2}+\frac{\left(M_{1}-M_{2}\right) a^{2}\left(r^{2}-2 b^{2} \log \frac{r}{b}-b^{2}\right)}{2 D_{p}\left[\left(1-v_{p}\right) b^{2}+\left(1+v_{p}\right) a^{2}\right]}, \quad(a \leq r \leq b) \text {. }
$$

Here, $M_{1}$ and $M_{2}$ are two intermediate bending moments, of which

$$
M_{1}=\frac{p}{16}\left[\left(1+v_{p}\right) b^{2}-\left(3+v_{p}\right) a^{2}\right]
$$


Similar to the case of the deflection induced by the piezoelectric effect, the continuity of the deflection at $r=a$ is already satisfied in Eqs. (3.49-3.50). And the continuity condition of the slope of the deflection at $r=a$ can be used to determine the other moment $M_{2}$. Due to its complexity, the equation of $M_{2}$ is not listed here.

\subsection{DEFLECTION OF RING-TYPE BENDING ACTUATOR}
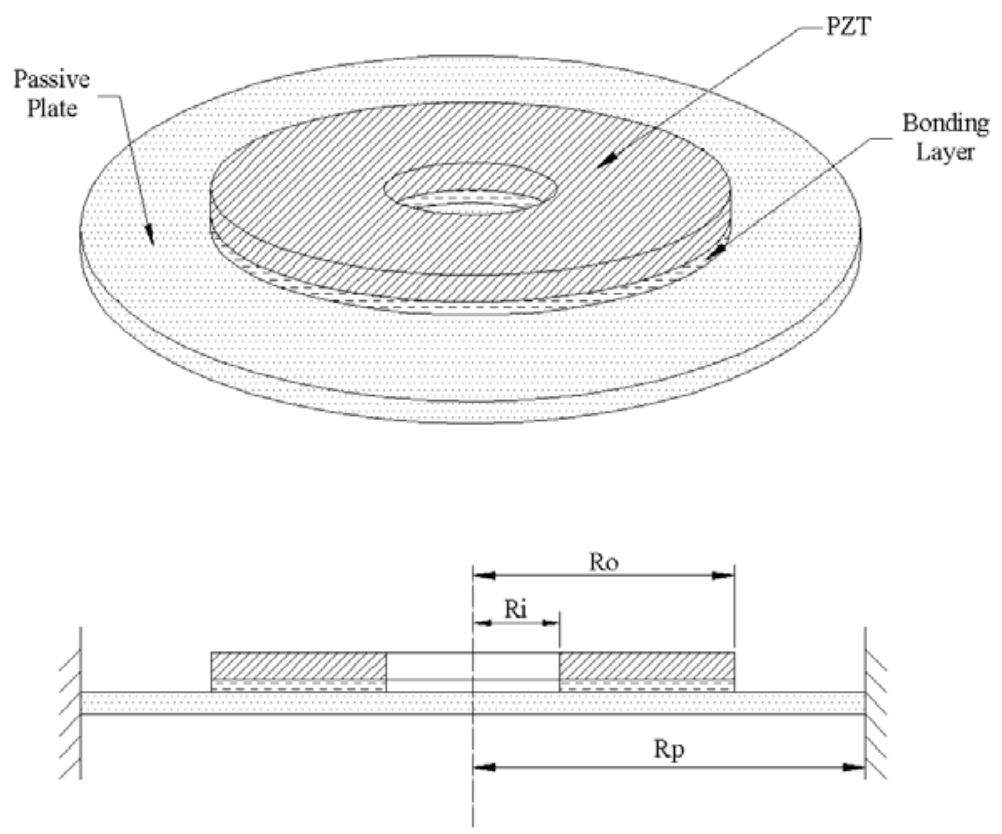

Figure 34 Schematic of the PZT ring-type bending actuator model

The schematic of the ring-type bending mode actuator and its model is shown in Fig. 34. It also consists of three layers: PZT ring, bonding layer and passive plate. The same assumptions used in the modeling of the disk-type bending actuator can be applied here as well. Both the applied voltage and the pressure difference can cause the actuator to bend. 


\section{Deflection caused by the applied voltage}

Now for a close approximation, assuming the whole system is linear, the deflection of the ringtype bending actuator can be obtained by subtracting the deflection of a smaller disk-type bending actuator from the deflection of a larger disk-type bending actuator. The radius of the smaller disk-type actuator is equal to the inner radius of the ring-type actuator and the radius of the larger disk-type bending actuator is equal to the outer radius of the ring-type bending actuator. Therefore, the deflection equations for the ring-type bending actuator can be written as,

$$
\begin{aligned}
& w_{1}(r)=\frac{M_{0}\left[\left(R_{p}^{2}-R_{o}^{2}\right)\left(R_{o}^{2}-r^{2}\right)+R_{o}^{2}\left(R_{o}^{2}-2 R_{p}^{2} \log \frac{R_{o}}{R_{p}}-R_{p}^{2}\right)\right]}{2\left\{D_{p}\left[\left(1+v_{p}\right) R_{o}^{2}+\left(1-v_{p}\right) R_{p}^{2}\right]+D_{e}\left(1+v_{e}\right)\left(R_{p}^{2}-R_{o}^{2}\right)\right\}} \\
& M_{0}\left[\left(R_{p}^{2}-R_{i}^{2}\right)\left(R_{i}^{2}-r^{2}\right)+R_{i}^{2}\left(R_{i}^{2}-2 R_{p}^{2} \log \frac{R_{i}}{R_{p}}-R_{p}^{2}\right)\right] \\
& -\frac{\left[D_{p}\left[\left(1+v_{p}\right) R_{i}^{2}+\left(1-v_{p}\right) R_{p}^{2}\right]+D_{e}\left(1+v_{e}\right)\left(R_{p}^{2}-R_{i}^{2}\right)\right\}}{2}, \quad\left(0 \leq r \leq R_{i}\right) . \\
& w_{2}(r)=\frac{M_{0}\left[\left(R_{p}^{2}-R_{o}^{2}\right)\left(R_{o}^{2}-r^{2}\right)+R_{o}^{2}\left(R_{o}^{2}-2 R_{p}^{2} \log \frac{R_{o}}{R_{p}}-R_{p}^{2}\right)\right]}{2\left\{D_{p}\left[\left(1+v_{p}\right) R_{o}^{2}+\left(1-v_{p}\right) R_{p}^{2}\right]+D_{e}\left(1+v_{e}\right)\left(R_{p}^{2}-R_{o}^{2}\right)\right\}} \\
& -\frac{M_{0} R_{i}^{2}\left(r^{2}-2 R_{p}^{2} \log \frac{r}{R_{p}}-R_{p}^{2}\right)}{2\left\{D_{p}\left[\left(1+v_{p}\right) R_{i}^{2}+\left(1-v_{p}\right) R_{p}^{2}\right]+D_{e}\left(1+v_{e}\right)\left(R_{p}^{2}-R_{i}^{2}\right)\right\}}, \quad\left(R_{i} \leq r \leq R_{o}\right) . \\
& w_{3}(r)=\frac{M_{0} R_{o}^{2}\left(r^{2}-2 R_{p}^{2} \log \frac{r}{R_{p}}-R_{p}^{2}\right)}{2\left\{D_{p}\left[\left(1+v_{p}\right) R_{o}^{2}+\left(1-v_{p}\right) R_{p}^{2}\right]+D_{e}\left(1+v_{e}\right)\left(R_{p}^{2}-R_{o}^{2}\right)\right\}} \\
& -\frac{M_{0} R_{i}^{2}\left(r^{2}-2 R_{p}^{2} \log \frac{r}{R_{p}}-R_{p}^{2}\right)}{2\left\{D_{p}\left[\left(1+v_{p}\right) R_{i}^{2}+\left(1-v_{p}\right) R_{p}^{2}\right]+D_{e}\left(1+v_{e}\right)\left(R_{p}^{2}-R_{i}^{2}\right)\right\}}, \quad\left(R_{o} \leq r \leq R_{p}\right) .
\end{aligned}
$$


Here, $M_{0}$ is determined by Eq. (3.42). $R_{i}$ and $R_{o}$ represent the inner and outer radius of the PZT ring. $R_{p}$ represents the radius of the passive plate. The meanings of other parameters are the same as introduced in the analysis of the disk-type bending actuator.

Therefore the whole deflection of the ring-type bending actuator is,

$$
w(r)=\left\{\begin{array}{lc}
w_{1}(r), \quad 0 \leq r \leq R_{i} ; \\
w_{2}(r), \quad R_{i} \leq r \leq R_{o} ; \\
w_{3}(r), \quad R_{o} \leq r \leq R_{p} .
\end{array}\right.
$$

\section{Deflection caused by the pressure}

Similar to the case of the disk-type actuator, the deflection induced by mechanical pressure can be determined by using the superposition method. Combining the established equations from [50] together, the deflections for the three different sections are,

$$
\begin{aligned}
& w_{4}(r)=w_{5}\left(R_{i}\right)+\frac{p\left(R_{i}^{2}-r^{2}\right)}{64 D_{p}}\left(\frac{5+v_{p}}{1+v_{p}} R_{i}^{2}-r^{2}\right)+\frac{M_{1}}{2 D_{p}\left(1+v_{p}\right)}\left(R_{i}^{2}-r^{2}\right), \quad\left(0 \leq r \leq R_{i}\right) . \\
& w_{5}(r)=w_{6}\left(R_{o}\right)+\frac{p\left(R_{o}^{2}-r^{2}\right)}{64 D_{e}}\left(\frac{5+v_{e}}{1+v_{e}} R_{o}^{2}-r^{2}\right)+\frac{\left[R_{o}^{2} M_{2}-R_{i}^{2}\left(M_{1}-M^{\prime}\right)\right]\left(R_{o}^{2}-r^{2}\right)}{2\left(1+v_{e}\right) D_{e}\left(R_{o}^{2}-R_{i}^{2}\right)} \\
& -\frac{R_{o}^{2} R_{i}^{2}\left(M_{2}-M_{1}+M^{\prime}\right) \log \left(\frac{r}{R_{o}}\right)}{\left(1-v_{e}\right) D_{e}\left(R_{o}^{2}-R_{i}^{2}\right)}, \quad\left(R_{i} \leq r \leq R_{o}\right) . \\
& w_{6}(r)=\frac{p}{64 D_{p}}\left(R_{p}^{2}-r^{2}\right)^{2}+\frac{\left(M^{\prime \prime}-M_{2}\right) R_{o}^{2}\left(r^{2}-2 R_{p}^{2} \log \frac{r}{R_{p}}-R_{p}^{2}\right)}{2 D_{p}\left[\left(1-v_{p}\right) R_{p}^{2}+\left(1+v_{p}\right) R_{o}^{2}\right]}, \quad\left(R_{o} \leq r \leq R_{p}\right) .
\end{aligned}
$$

Here, $M^{\prime}$ and $M^{\prime \prime}$ are two intermediate bending moments, of which

$$
\begin{gathered}
M^{\prime}=\frac{p}{16}\left(3+v_{e}\right)\left(R_{o}^{2}-R_{i}^{2}\right) \\
M^{\prime \prime}=\frac{p}{16}\left[R_{p}^{2}\left(1+v_{p}\right)-R_{o}^{2}\left(3+v_{p}\right)\right]
\end{gathered}
$$


The other two intermediate bending moments, $M_{1}$ and $M_{2}$, can be obtained by solving the continuity equations listed below.

$$
\left\{\begin{array}{l}
\left.\frac{d w_{4}(r)}{d r}\right|_{r=R_{i}}=\left.\frac{d w_{5}(r)}{d r}\right|_{r=R_{i}} \\
\left.\frac{d w_{5}(r)}{d r}\right|_{r=R_{o}}=\left.\frac{d w_{6}(r)}{d r}\right|_{r=R_{o}}
\end{array}\right.
$$

Note that the continuity condition of the deflection is already satisfied in Eqs. (3.56-3.58).

\subsection{VOLUME CHANGE, FLOW RATE AND POWER CONSUMPTION}

After the total deflection is determined, Eq. (3.3) can be used to calculate the volume change of the pump chamber.

For disk-type bending actuator,

$$
w(r)=w_{U}(r)+w_{p}(r)=\left\{\begin{array}{ll}
w_{1}(r), & 0 \leq r \leq a . \\
w_{2}(r), & a \leq r \leq b .
\end{array}+ \begin{cases}w_{3}(r), & 0 \leq r \leq a . \\
w_{4}(r), & a \leq r \leq b .\end{cases}\right.
$$

For ring-type bending actuator,

$$
w(r)=w_{U}(r)+w_{p}(r)=\left\{\begin{array}{cc}
w_{1}(r), & 0 \leq r \leq R_{i} . \\
w_{2}(r), & R_{i} \leq r \leq R_{o} \\
w_{3}(r), & R_{o} \leq r \leq R_{p} .
\end{array} . \quad\left\{\begin{array}{cc}
w_{4}(r), & 0 \leq r \leq R_{i} . \\
w_{5}(r), & R_{i} \leq r \leq R_{o} . \\
w_{6}(r), & R_{o} \leq r \leq R_{p} .
\end{array}\right.\right.
$$

Based on the previous derivations, it is known that the deflection caused by the piezoelectric effect, $w_{U}(r)$, is proportional to the applied voltage while the deflection induced by the mechanical pressure, $w_{p}(r)$, is proportional to the pressure difference. Therefore it can be easily shown that after the substitution of them into Eq. (3.3), the volume change can be expressed as,

$$
\Delta V=k_{U} U+k_{p} p
$$


Both proportional coefficients, $k_{U}$ and $k_{p}$ are only determined by the dimensions and material properties of the pump actuator. The total volume change of the pump chamber consists of two parts: one is proportional to the applied voltage and the other is proportional to the pressure difference. In actual pump applications, the applied voltage can be easily controlled while the pressure inside the pump chamber is coupled with the deformation of the pump actuator and the flow resistance of the whole system. Numerical iteration methods can be used to determine the volume change of the pump chamber precisely.

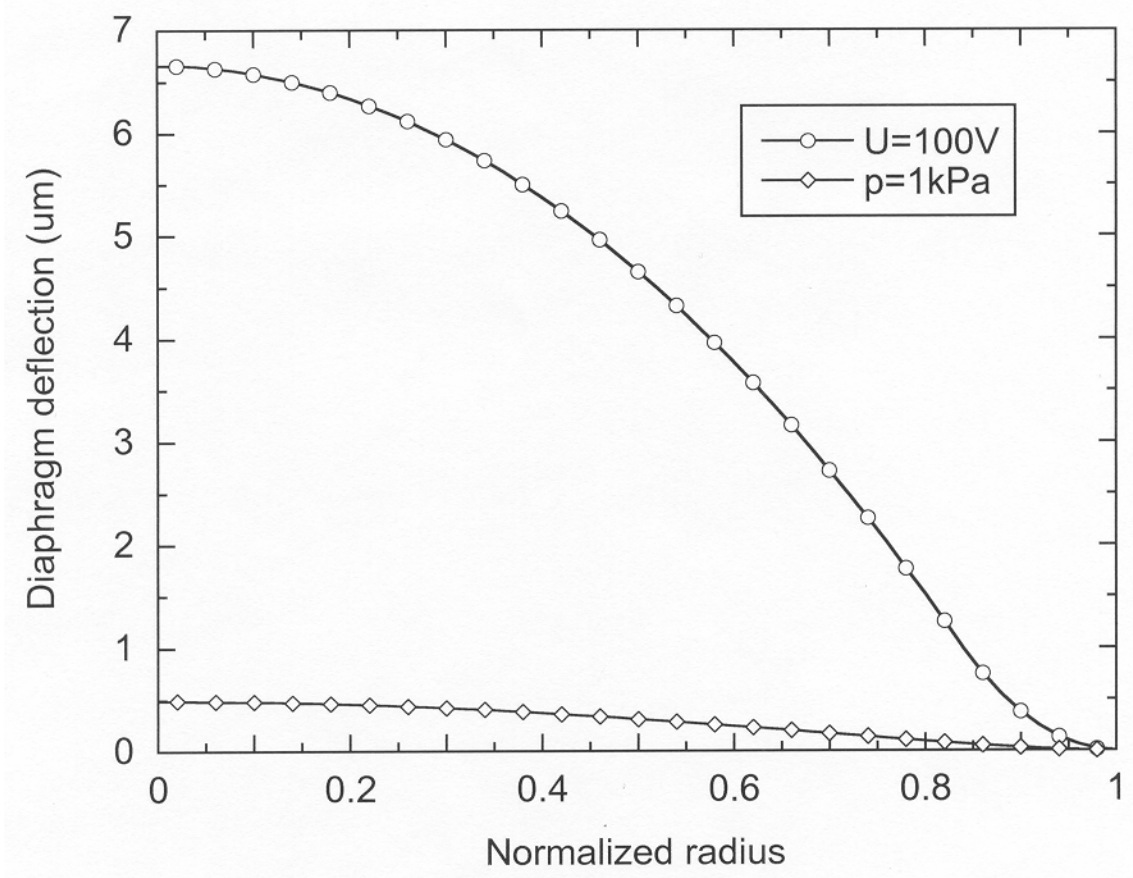

Figure 35 Comparison of the diaphragm deflections under 100 volts and $1 \mathrm{kPa}$ separately

If the pressure difference exerted on the pump actuator is only in the range of several $\mathrm{kPa}$, the deflection caused by the pressure difference will be very small. Fig. 35 shows the comparison between the deflection under 100 volt of applied voltage and the deflection under $1 \mathrm{kPa}$ of pressure difference for the disk-type bending actuator. The dimensions and material properties of the pump actuator used for this calculation are listed in Table 3. Under such circumstance, the effect of the pressure difference on the volume change can be neglected and an analytical 
solution can be obtained for the flow rate. To form a continuous flow, an alternating voltage has to be applied to the pump bending actuator. If the driving frequency is far lower than the resonance frequency of the pump actuator, $U$ can be substituted by $U_{m} \sin (\omega t)$ in the equations derived previously. $U_{m}$ is the amplitude of the sinusoidal voltage applied on the piezoelectric plate and $\omega$ is the angular frequency.

Table 3 Dimensions and material properties of the PZT disk-type bending actuator

\begin{tabular}{|c|c|c|c|}
\hline & Piezoelectric Disk & Bonding Layer & Passive Plate \\
\hline Materials & PZT-5H & Conductive Epoxy & Stainless Steel \\
\hline Diameter (mm) & 20 & 20 & 24 \\
\hline Thickness (mm) & 0.2 & 0.02 & 0.25 \\
\hline Young's Modulus (Pa) & $6.2 \times 10^{10}$ & $5.17 \times 10^{9}$ & 0.3 \\
\hline Poisson's Ratio & 0.31 & 0.3 & \\
\hline$d_{31}(\mathrm{~m} / \mathrm{V})$ & $-3.2 \times 10^{-10}$ & & \\
\hline$\varepsilon_{33}^{T}(\mathrm{~F} / \mathrm{m})$ & $2.856 \times 10^{-8}$ & & \\
\hline
\end{tabular}

To estimate the net flow rate of the pump, the modeling of the nozzle/diffuser is also necessary. As mentioned before, these nozzle/diffuser elements can direct flow from inlet to outlet. They are geometrically designed to have a lower pressure loss in one direction than in the opposite direction for the same flow velocity. The characteristic of nozzle/diffuser element can be described as follows,

$$
\Delta p=\frac{1}{2} \rho v^{2} \xi
$$


Here, $\Delta p$ is the pressure loss through the nozzle/diffuser element, $\rho$ is the density of the fluid, $v$ is the velocity of the fluid, and $\xi$ is the pressure loss coefficient. And it can also be expressed in the form of flow rate $Q$,

$$
Q=C \sqrt{\Delta p}
$$

Here, $C$ is called conductivity coefficient. Two different flow directions correspond to two different $C$ values. One is higher than the other. The conductivity coefficient in positive direction is represented by $C_{H}$ and the conductivity coefficient in negative direction is represented by $C_{L}$. Accordingly, there are also two different $\xi$ values: the pressure loss coefficient in positive direction is represented by $\xi_{\text {positive }}$ and the conductivity coefficient in negative direction is represented by $\xi_{\text {negative }}$.

As mentioned before, it is assumed that the effect of the pressure difference on the volume change is negligible and thus only the volume change induced by applied voltage is considered. Also assume that there is no pressure variance throughout each chamber so that the pressures of the pump chamber and the fuel chamber can be represented as $p_{p}$ and $p_{f}$ respectively. Given these assumptions, the equations derived by Ullmann [42] can be used to obtain the analytical solutions for the fuel flow rate and the pressure of the pump chamber. The pressure of the fuel chamber is set to be the atmospheric pressure.

(1) Pump mode: $p_{p}>p_{f}$

$$
\begin{array}{r}
Q_{1}=\frac{C_{L}}{C_{H}+C_{L}} k_{U} U_{m} \omega \cos (\omega t) \\
Q_{2}=\frac{C_{H}}{C_{H}+C_{L}} k_{U} U_{m} \omega \cos (\omega t)
\end{array}
$$




$$
p_{p}=p_{f}+\left[\frac{k_{U} U_{m} \omega \cos (\omega t)}{C_{H}+C_{L}}\right]^{2}
$$

(2) Supply mode: $p_{p}<p_{f}$

$$
\begin{gathered}
Q_{1}=-\frac{C_{H}}{C_{H}+C_{L}} k_{U} U_{m} \omega \cos (\omega t) \\
Q_{2}=-\frac{C_{L}}{C_{H}+C_{L}} k_{U} U_{m} \omega \cos (\omega t) \\
p_{p}=p_{f}-\left[\frac{k_{U} U_{m} \omega \cos (\omega t)}{C_{H}+C_{L}}\right]^{2}
\end{gathered}
$$

The average net flow rate is given by

$$
\bar{Q}=\frac{k_{U} U_{m} \omega}{\pi} \frac{C_{H}-C_{L}}{C_{H}+C_{L}}=\frac{k_{U} U_{m} \omega}{\pi} \frac{\eta^{\frac{1}{2}}-1}{\eta^{\frac{1}{2}}+1}
$$

Here,

$$
\eta=\frac{\xi_{\text {negative }}}{\xi_{\text {positive }}}=\left(\frac{C_{H}}{C_{L}}\right)^{2}
$$

Since the electrical power used to drive the micropump comes from the fuel cell itself, it is necessary to estimate how much energy the micropump needs to consume. Electrically the bending piezoelectric actuator behaves like a planar capacitor. The electric capacitance under the given mechanical boundary condition is very complicated. In a similar case [52], the capacitance of a circular disk-type PZT actuator with clamped edges has been given by

$$
C=\frac{\varepsilon_{33}^{T} \pi a^{2}}{h_{p z t}}\left\{1-\frac{2}{1-v}\left[1-\frac{3\left(s_{11}^{E}\right)^{2} s_{p} h_{p}^{2} h_{p z t}\left(h_{p}+h_{p z t}\right)^{2}}{S_{h} B_{31}}\right] K_{31}^{2}\right\}
$$


Here, $\varepsilon_{33}^{T}$ is the permittivity of the PZT disk. $s_{11}^{E}$ is the elastic compliance of the PZT disk at constant electric field and $s_{p}$ is the elastic compliance of the passive layer. $K_{31}$ is the electromechanical coupling coefficient of the PZT disk. $v$ is the Poisson's ratio. In [52] it is assumed that the Poisson's ratio of the PZT disk is the same as that of the passive plate and the effect of the bonding layer on the capacitance is neglected. The equations for the other two parameters are:

$$
S_{h}=h_{p z t} s_{p}+h_{p} s_{11}^{E}, \quad B_{31}=h_{p z t}^{4} s_{p}^{2}+4 s_{11}^{E} s_{p} h_{p} h_{p z t}^{3}+6 s_{11}^{E} s_{p} h_{p}^{2} h_{p z t}^{2}+4 s_{11}^{E} s_{p} h_{p}^{3} h_{p z t}+h_{p}^{4}\left(s_{11}^{E}\right)^{2} .
$$

The term outside the parenthesis of Eq. (3.75) is actually the capacitance with free boundary condition. Using the dimensions and material properties of the piezoelectric actuator listed in Table 3 for calculation, it is found that the capacitance with clamped boundary condition is about 30 percent less than the capacitance with free boundary condition. Although the boundary condition of the pump actuator in this study is not exactly the same as that of [52], the results of two cases should be close. For approximation the capacitance with free boundary condition is used here because the resultant estimation of the pump power consumption will be larger, which should be safer for the system design. The capacitance with free boundary condition is given by

$$
C_{0}=\frac{\text { Permittivity } \times \text { Area }}{\text { Dis } \tan c e}
$$

For the disk-type bending actuator,

$$
C_{0}=\frac{\varepsilon_{33}^{T} \pi a^{2}}{h_{p z t}}
$$

For the ring-type bending actuator,

$$
C_{0}=\frac{\varepsilon_{33}^{T} \pi\left(R_{o}^{2}-R_{i}^{2}\right)}{h_{p z t}}
$$


Suppose the applied voltage is sinusoidal, that is

$$
U(t)=U_{m} \sin (\omega t)
$$

The current is then,

$$
i(t)=C_{0} \frac{d U(t)}{d t}
$$

So the instantaneous power consumption is given by

$$
W_{\text {elec }}=i(t) U(t)=\frac{1}{2} \omega C_{0} U_{m}^{2} \sin (2 \omega t)
$$

It should be noted that as a capacitor, the piezoelectric actuator would store a large portion of the input electrical energy; therefore only part of the input energy can be converted into output mechanical energy for fuel delivery. The stored energy will remain in the driving circuit and will be used in next driving cycle. For the worst case, the maximum value of $W_{\text {elec }}$ is chosen to estimate the performance of the whole fuel cell system. Combining Eq. (3.73) and Eq. (3.82) by eliminating $U_{m}$, we have

$$
\max W_{\text {elec }}=\frac{\pi C_{0}}{4 k_{U}^{2} f}\left(\frac{\eta^{\frac{1}{2}}+1}{\eta^{\frac{1}{2}}-1}\right)^{2} \bar{Q}^{2}
$$

Here, $f$ is the frequency of the driving voltage.

As shown in Eq. (3.83), the maximum power consumption is proportional to the capacitance, $C_{0}$, and the square of the average net flow rate, $\bar{Q}$. It is also inversely proportional to the driving frequency, $f$, and the square of $k_{U}$. The effect of the nozzle/diffuser element is represented by the coefficient, $\eta$. The larger this coefficient is, the less the power consumption is. Both $C_{0}$ and $k_{U}$ depend on the dimensions and material properties of the pump actuator. 


\subsection{SUMMARY}

In this chapter, first the theoretical equations have been established to calculate the deflection of the pump actuator. From the deflection, the volume change of the whole pump chamber is determined. Taking the characteristic of nozzle/diffuser into account, the pump flow rate can be determined under some simplifications. The power consumption of the micropump is estimated by considering the pump actuator as a capacitor. Finally, the relationships between the design parameters (material properties, dimensions) and the operation parameters (driving voltage, driving frequency, flow rate, and power consumption) are established. These relationships can help us design a micropump to meet the requirements of the fuel cell system. In the following chapters, the effects of the material properties and the dimension on the performance of the micropump will be investigated to find optimized design parameters. Also combining these theoretical results with selected fuel cell experimental data, the viability of the DMFC power system integrated with the micropump will be verified. 


\subsection{OPTIMIZATION OF MICROPUMP DESIGN}

Both the passive layer and the active layer of the micropump can be made of various materials. Also the thickness and the radius of each layer may be different. It is possible to optimize the micropump design based on the theoretical equations derived in the previous chapter. There are two objectives that can be used for optimization. One is the average net flow rate. If the driving voltage and its frequency are fixed, based on Eq. (3.73) a larger $k_{U}$ is preferred so that a larger flow rate can be obtained. The other objective is the power consumption. It is shown in Eq. (3.83) that given the driving frequency and the flow rate, a larger $k_{U}^{2} / C_{0}$ is preferred so that the power required to drive the micropump is smaller. Both factors will be evaluated in the following analysis.

\subsection{DISK-TYPE BENDING ACTUATOR}

\subsubsection{Verification by numerical simulation and experimental measurement}

It is necessary to verify the analytical solutions since many assumptions and simplifications have been made during the derivation. Here both numerical and experimental results are used to compare with the theoretical results for the disk-type bending actuator. The numerical results are

obtained by using a commercial FEM package, ANSYS 6.1. Since the whole bending actuator is symmetric in circumferential direction, only a wedge-shaped part of it with fixing and symmetric 
boundary conditions is considered for the calculation [53]. A 3-D tetrahedral structural solid element type, Solid-92 is chosen to model the passive plate and a 3-D tetrahedral coupled-field solid element type, Solid-98 is chosen to model the PZT layer. The bonding layer is neglected. The total number of the elements in this model is 4938. The experimental data can be found available in [35]. The same dimensions and material properties listed in Table 4 are used for all the calculations here. The applied voltage is $50 \mathrm{~V}$.

Table 4 Dimensions and material properties of the PZT disk-type bending actuator in [35]

\begin{tabular}{|c|c|c|c|}
\hline & Piezoelectric Disk & Bonding Layer & Passive Plate \\
\hline Materials & PZT-5A & EPO-TEK H31 & Pyrex Glass \\
\hline Diameter (mm) & 5 & 5 & 6 \\
\hline Thickness (mm) & 0.2 & 0.02 & 0.5 \\
\hline Young's Modulus (Pa) & $6.7 \times 10^{10}$ & $5.17 \times 10^{9}$ & $6.275 \times 10^{10}$ \\
\hline Poisson's Ratio & 0.31 & 0.3 & 0.2 \\
\hline$d_{31}(\mathrm{~m} / \mathrm{V})$ & $-1.9 \times 10^{-10}$ & & \\
\hline$\varepsilon_{33}^{T}(\mathrm{~F} / \mathrm{m})$ & $1.5937 \times 10^{-8}$ & & \\
\hline
\end{tabular}

Fig. 36 shows the numerical results obtained by ANSYS and the maximum deflection appears at the center of the structure. Note that the deflection distribution is actually different along the thickness direction because the 3-D elements are used in the program. 
NODẢL SOLUTION

STEP $=1$

SUB $=1$

TIVE $=1$

$\mathrm{UZ}$

RSYS $=0$

$\mathrm{DNX}=.176 \mathrm{E}-06$

SMX $=.176 \mathrm{E}-06$
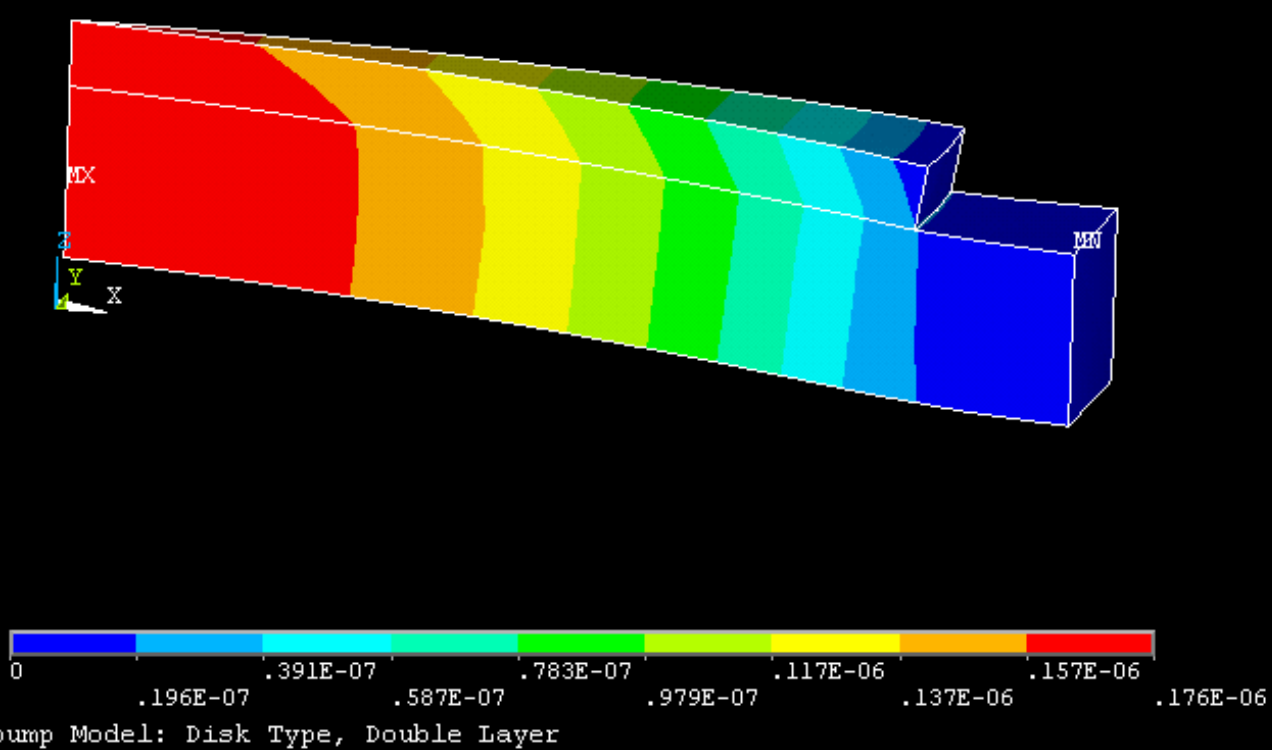

Micropump Model: Disk Type, Double Layer

Figure 36 Deformation results of the PZT disk-type bending actuator using ANSYS

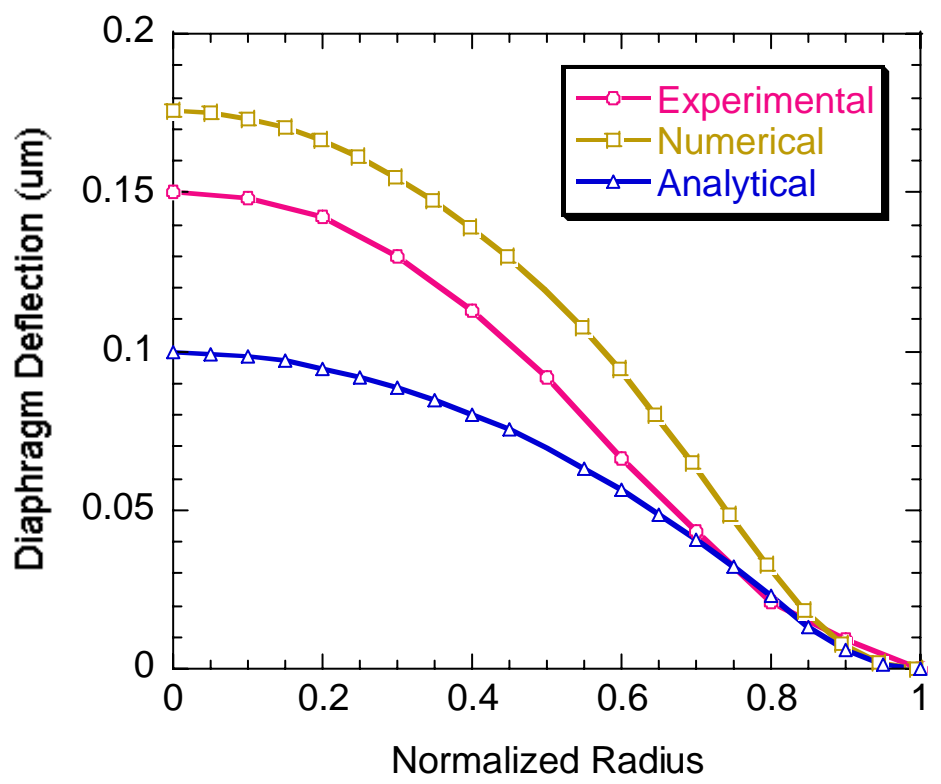

Figure 37 Comparison of the diaphragm deflections for the PZT disk-type bending actuator obtained by different methods $(\mathrm{U}=50 \mathrm{~V})$ 
Shown in Fig. 37 is the comparison of the deflection data obtained by three different methods. In general the three curves share the similar trend and the maximum deflection always appears at the center. However, there are some discrepancies. The theoretical deflection is the smallest while the numerical one is the largest. There are several possible reasons to explain the differences. First of all, the experimental conditions are somehow different from theoretical and numerical assumptions: the fabrication process may result in some residual stress or strain and the boundary conditions are not ideal fixed. Also the actual deformation of the bending actuator is three-dimensional, but the numerical result is two-dimensional and the theoretical result is only one-dimensional. Furthermore, there may be defections and nonuniformities in the materials used for experiments therefore the material properties won't be very accurate. Nonetheless, both numerical and theoretical methods should be able to provide fair estimations.

\subsubsection{Effects of material properties}

The effect of Young's modulus of both PZT disk and passive plate on the performance of the pump actuator is investigated. Shown in Fig. 38 is the variation of $k_{U}$ when changing the Young's modulus of the PZT disk (the other parameters are kept the same as listed in Table 4). It is found that the large the Young's modulus of the PZT disk, the large the $k_{U}$. Note that the unit of $k_{U}$ is $m^{3} / V$, which basically means how much volume change can be obtained by applying 1 volt of voltage across the PZT disk. As for the passive plate, the similar calculation can be done but the result (shown in Fig. 39) is opposite: the large the Young's modulus of the passive plate, the smaller the $k_{U}$. In both cases, Young's modulus is varying from $30 \mathrm{GPa}$ to $600 \mathrm{GPa}$, which covers almost all the possible pump fabrication materials such as glass, silicon, silicon nitride, 
and most metals. Since the capacitance is not related to the Young's modulus, the variation trend of $k_{U}^{2} / C_{0}$ should be the same as that of $k_{U}$. The Young's modulus of the PZT materials does not change a lot so Fig. 38 just shows the variation trend.

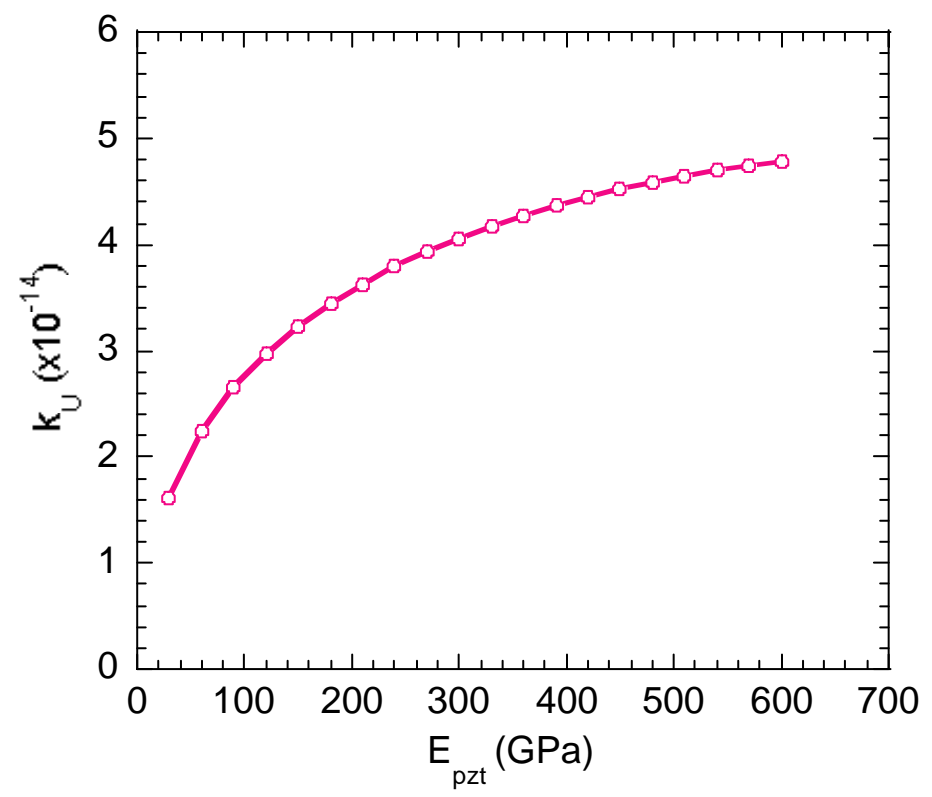

Figure 38 Relationship between the proportional coefficient $k_{U}$ and the PZT layer Young's modulus $E_{p z t}$

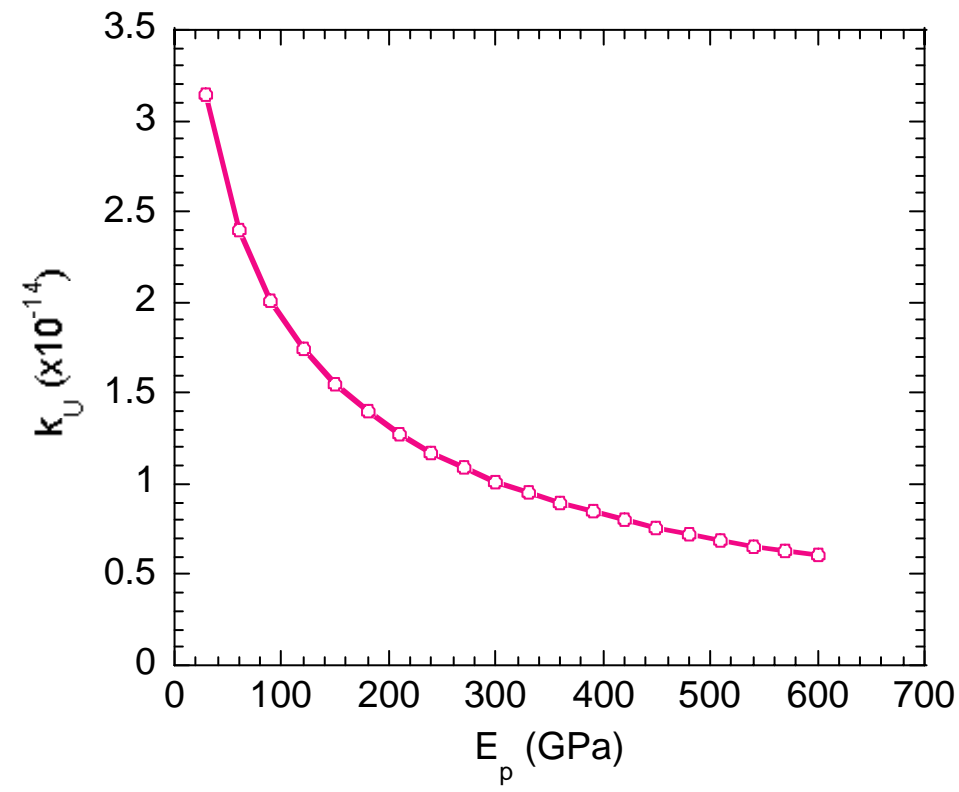

Figure 39 Relationship between the proportional coefficient $k_{U}$ and the passive plate Young's modulus $E_{p}$ 


\subsubsection{Effects of structure dimensions}

Other important factors that can affect the performance of the micropump are structure dimensions including both radius and thickness.

Fig. 40 gives the relationship among the PZT/passive plate radius ratio, $k_{U}$, and $k_{U}^{2} / C_{0}$. It is shown that for both $k_{U}$ and $k_{U}^{2} / C_{0}$ there exists an optimal PZT/passive plate radius ratio at which maximum value can be obtained. As for $k_{U}$, the optimal PZT/passive plate radius ratio is about 0.8. Since increasing the radius of PZT disk will increase the capacitance, the optimal radius ratio for $k_{U}^{2} / C_{0}$ is a little bit smaller and around 0.7. By fixing this PZT/passive plate radius ratio, it is found that increasing the radius of the passive plate will increase both $k_{U}$ and $k_{U}^{2} / C_{0}$. This is shown in Fig. 41.

As shown in Fig. 42, $k_{U}$ is decreasing when the thickness of the PZT disk is increasing. This is because the electric field applied to the PZT disk is increasing, but the electric field cannot exceed a certain limit or the PZT materials will be depolarized. Different from the case of $k_{U}$, there exists an optimal thickness at which $k_{U}^{2} / C_{0}$ is the largest. This is because the smaller the PZT disk thickness is, the larger the capacitance is. The value of this optimal thickness is about $0.25 \mathrm{~mm}$ if the other parameters are as listed in Table 4.

Now fixing the thickness of PZT disk, the relationship between the PZT/passive plate thickness ratios and $k_{U}, k_{U}^{2} / C_{0}$ can be obtained. From Fig. 43, it is shown that the optimal thickness ratio exits for both $k_{U}$ and $k_{U}^{2} / C_{0}$. Since the capacitance is not related to the thickness of the passive plate, the values of both optimal thickness ratios are the same and about 0.4 in this case. 


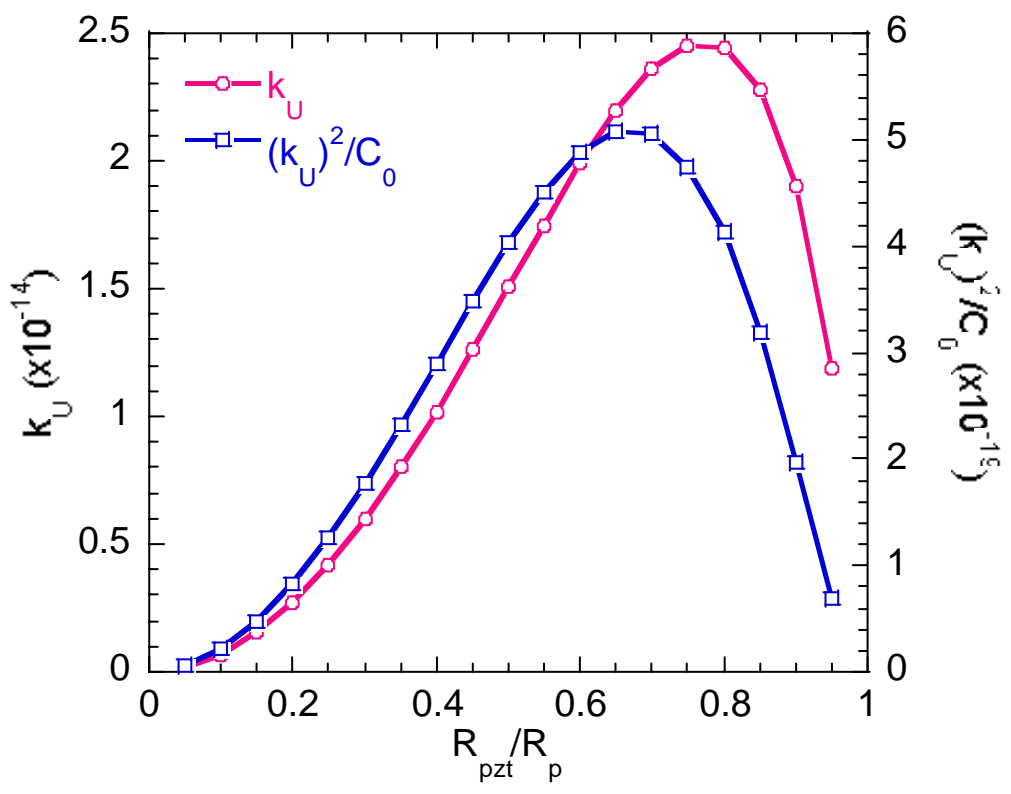

Figure 40 Coefficient $k_{U}$ and $k_{U}^{2} / C_{0}$ at different $\mathrm{PZT} /$ passive plate radius ratio

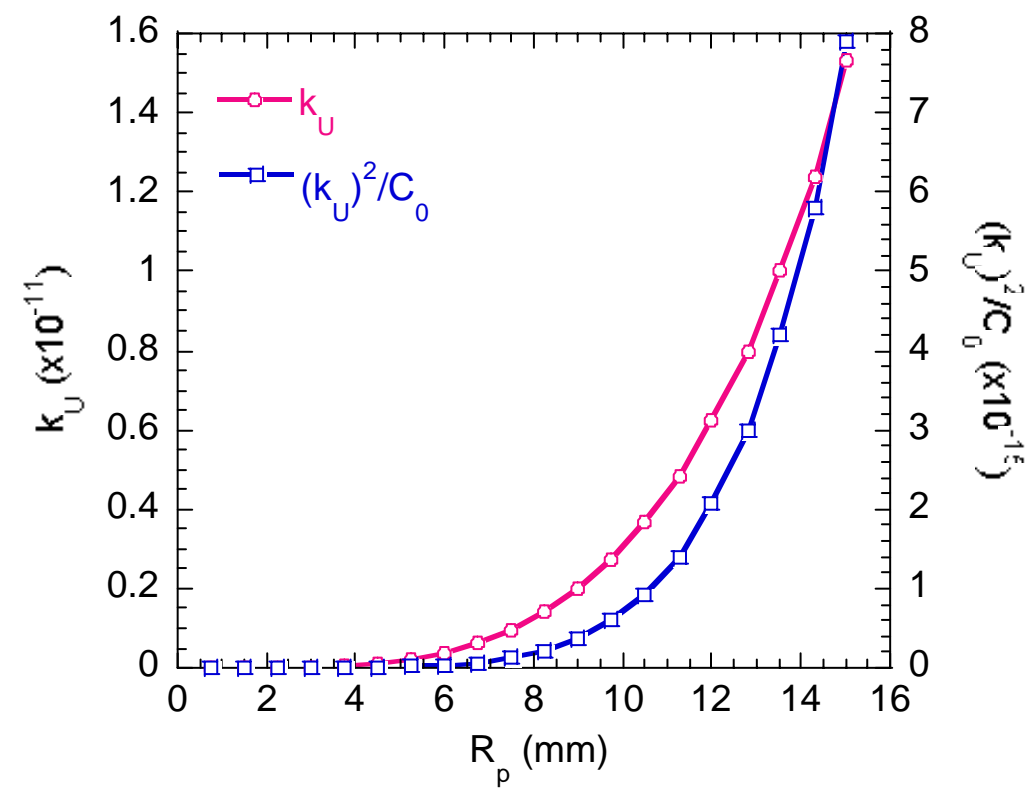

Figure 41 Coefficient $k_{U}$ and $k_{U}^{2} / C_{0}$ at different passive plate radius with fixed PZT/passive plate radius ratio 


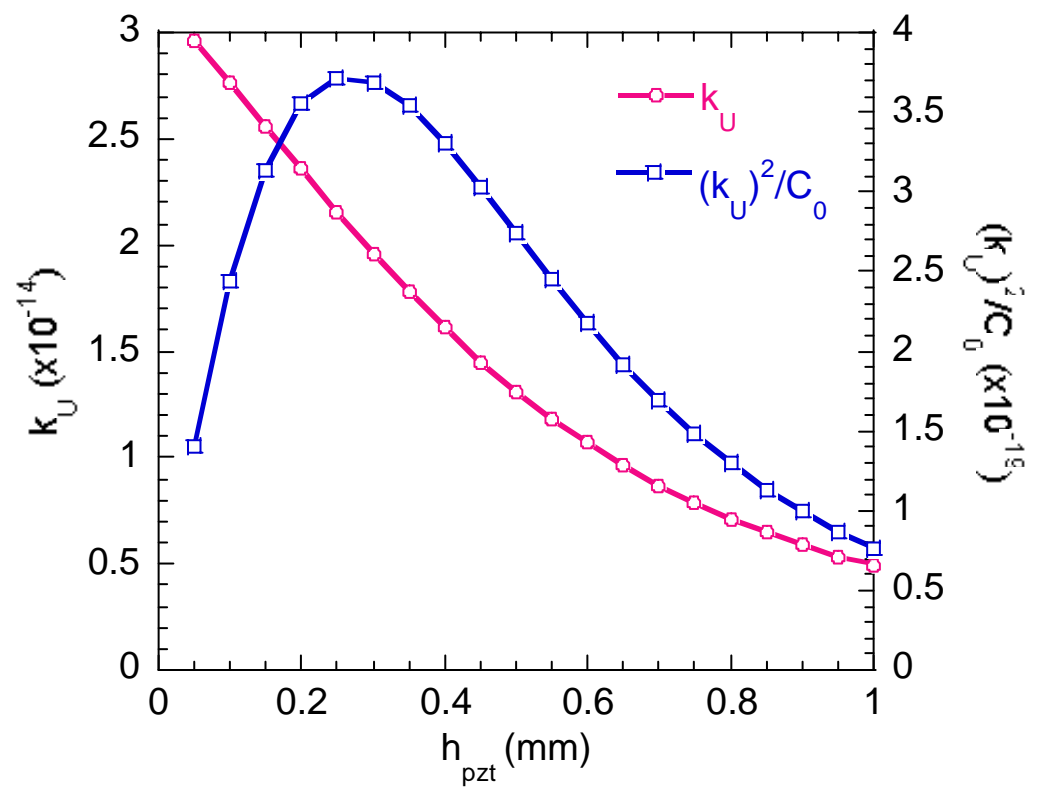

Figure 42 Coefficient $k_{U}$ and $k_{U}^{2} / C_{0}$ at different PZT layer thickness

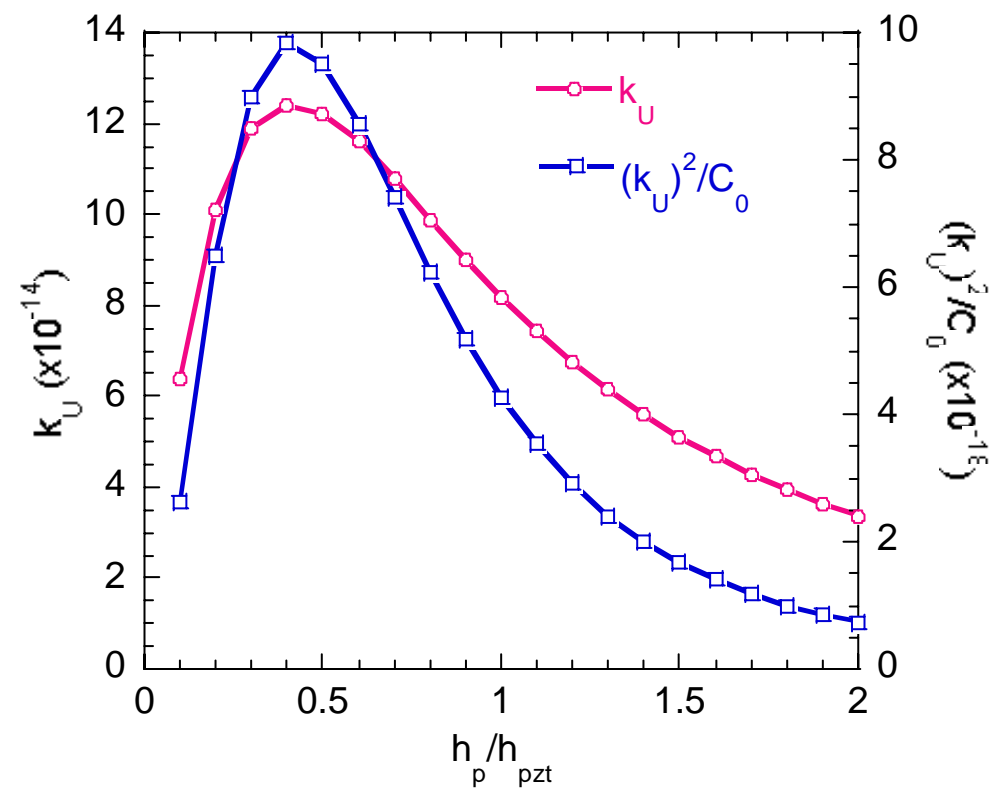

Figure 43 Coefficient $k_{U}$ and $k_{U}^{2} / C_{0}$ at different passive/PZT layer thickness ratio with fixed PZT layer thickness 


\subsection{RING-TYPE BENDING ACTUATOR}

\subsubsection{Verification by numerical simulation and experimental measurement}

Table 5 Dimensions and material properties of the PZT ring-type bending actuator

\begin{tabular}{|c|c|c|c|}
\hline & Piezoelectric Ring & Bonding Layer & Passive Plate \\
\hline Materials & PZT-5H & Conductive Epoxy & Stainless Steel \\
\hline Inner Diameter (mm) & 6.3 & 6.3 & 25.4 \\
\hline Outer Diameter (mm) & 19.11 & 19.11 & 0.254 \\
\hline Thickness (mm) & 0.32 & 0.056 & $1.95 \times 10^{11}$ \\
\hline Young's Modulus (Pa) & $6.2 \times 10^{10}$ & $5.17 \times 10^{9}$ & 0.3 \\
\hline Poisson’s Ratio & 0.31 & 0.3 & \\
\hline$d_{31}(\mathrm{~m} / \mathrm{V})$ & $-3.2 \times 10^{-10}$ & & \\
\hline$\varepsilon_{33}^{T}(\mathrm{~F} / \mathrm{m})$ & $2.856 \times 10^{-8}$ & & \\
\hline
\end{tabular}

For the ring-type bending actuator, numerical and experimental methods are also used to verify the theoretical model. As same as the case of disk-type bending actuator, the numerical results are obtained by using the commercial FEM package, ANSYS 6.1 to calculate a wedge-shaped part of the pump actuator with fixing and symmetric boundary conditions. A 3-D tetrahedral structural solid element type, Solid-92 is chosen to model the passive plate and a 3-D tetrahedral coupled-field solid element type, Solid-98 is chosen to model the PZT layer. The bonding layer is also neglected. The total number of the elements in this model is 2753. The experimental data 
are measured from a ring-type bending actuator fabricated in our lab. The dimensions and material properties of this lab-made actuator are listed in Table 5. The applied voltage is $100 \mathrm{~V}$. Fig. 44 shows the numerical results obtained by ANSYS and the maximum deflection also appears at the center of the structure. And the deflection distribution is actually two-dimensional. The comparison of the deflection data obtained by three different methods is shown in Fig. 45. For both numerical results and experimental results, the maximum deflection appears at the center of the pump actuator. Theoretical results are closer to the experimental results although the maximum deflection doesn't appear at the center. This probably means the linear approximation during the theoretical derivation doesn't fit well with the real case. Also note that due to the imperfect clamping at the outer edge of the lab-made pump actuator, the experimental results don't converge to zero at the boundary. Still both numerical and theoretical methods should be able to provide fair estimations. 


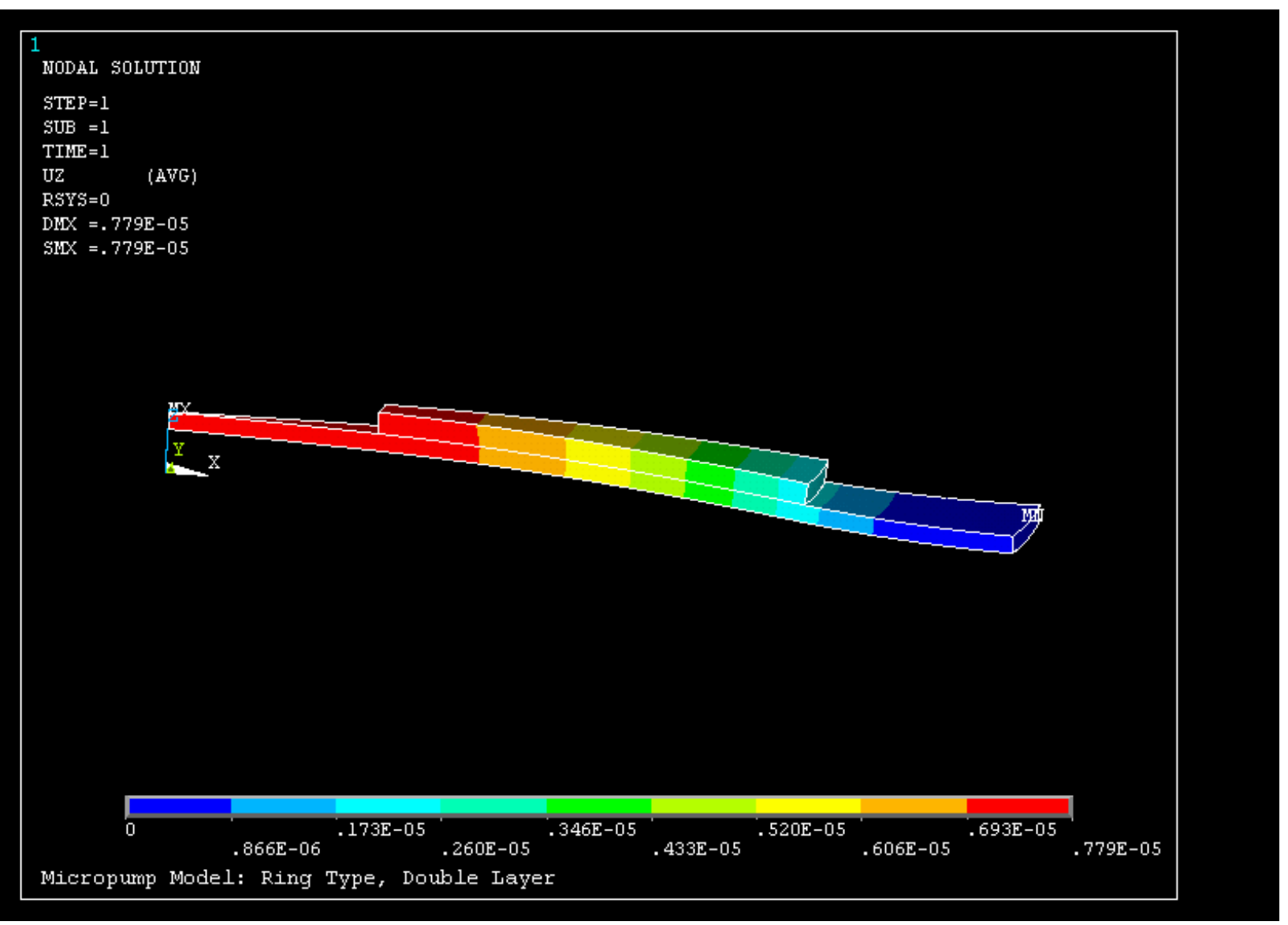

Figure 44 Deformation results of the PZT ring-type bending actuator using ANSYS

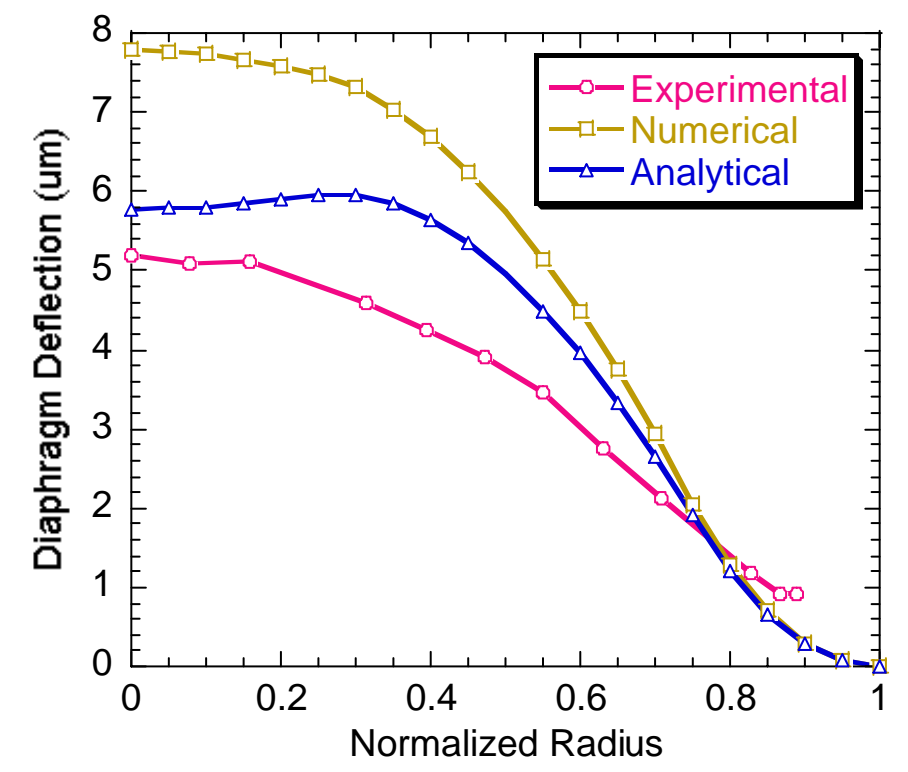

Figure 45 Comparison of the diaphragm deflections for the PZT ring-type bending actuator obtained by different methods $(\mathrm{U}=\mathbf{1 0 0 \mathrm { V }})$ 


\subsubsection{Effects of material properties}

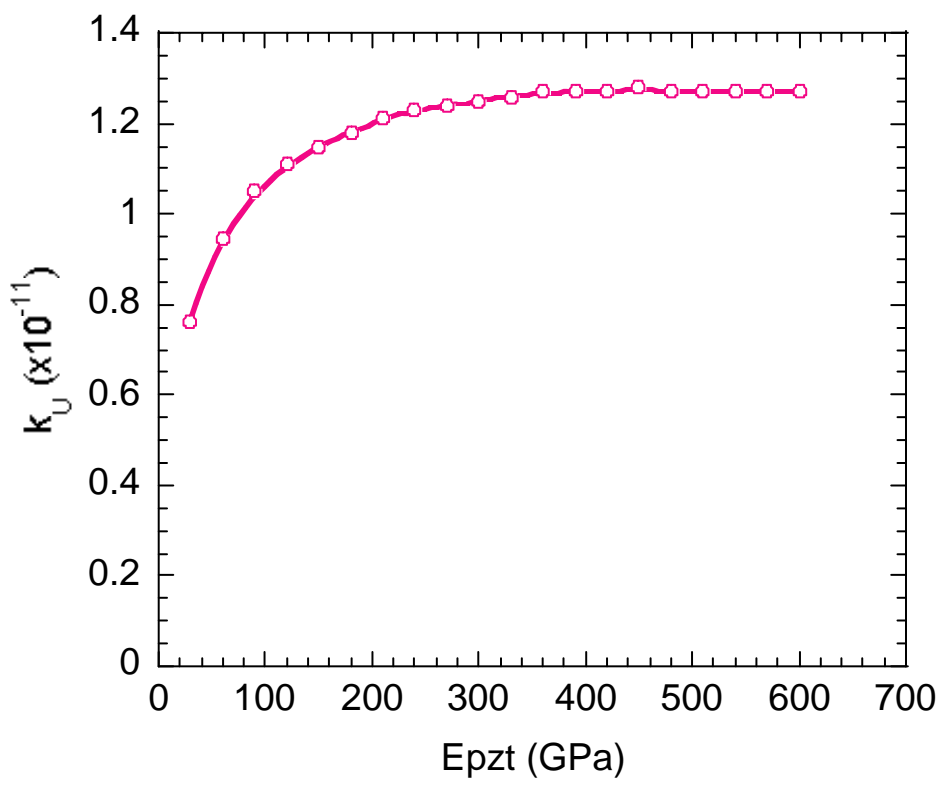

Figure 46 Relationship between the proportional coefficient $k_{U}$ and the PZT layer Young's modulus $E_{p z t}$

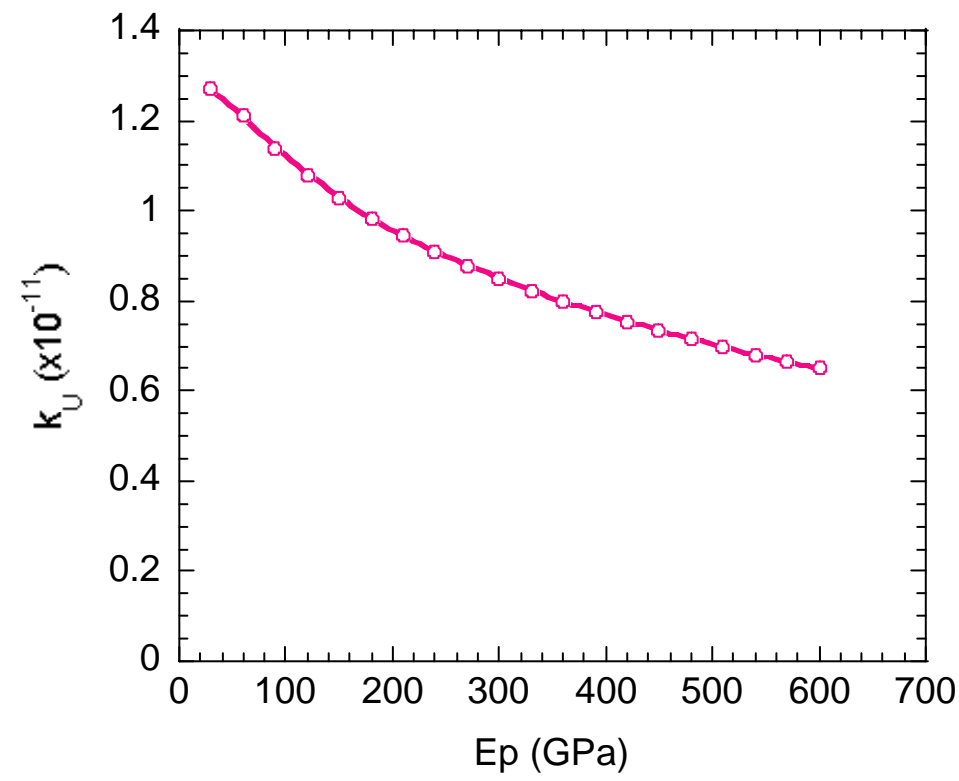

Figure 47 Relationship between the proportional coefficient $k_{U}$ and the passive plate Young's modulus $E_{p}$ 
Fig. 46 and Fig. 47 illustrate how the material properties affect the performance of the ring-type bending actuator. The larger the Young's modulus of the PZT disk is, the higher the $k_{U}$ is. After the Young's modulus of the PZT disk exceeds $400 \mathrm{GPa}, k_{U}$ becomes stable. The opposite case is observed when changing the Young's modulus of the passive plate. Increasing the Young's modulus of the passive plate will reduce $k_{U}$. In addition, because there is no connection between the capacitance and the Young's modulus, the variation trend of $k_{U}^{2} / C_{0}$ should be the same as that of $k_{U}$.

\subsubsection{Effects of structure dimensions}

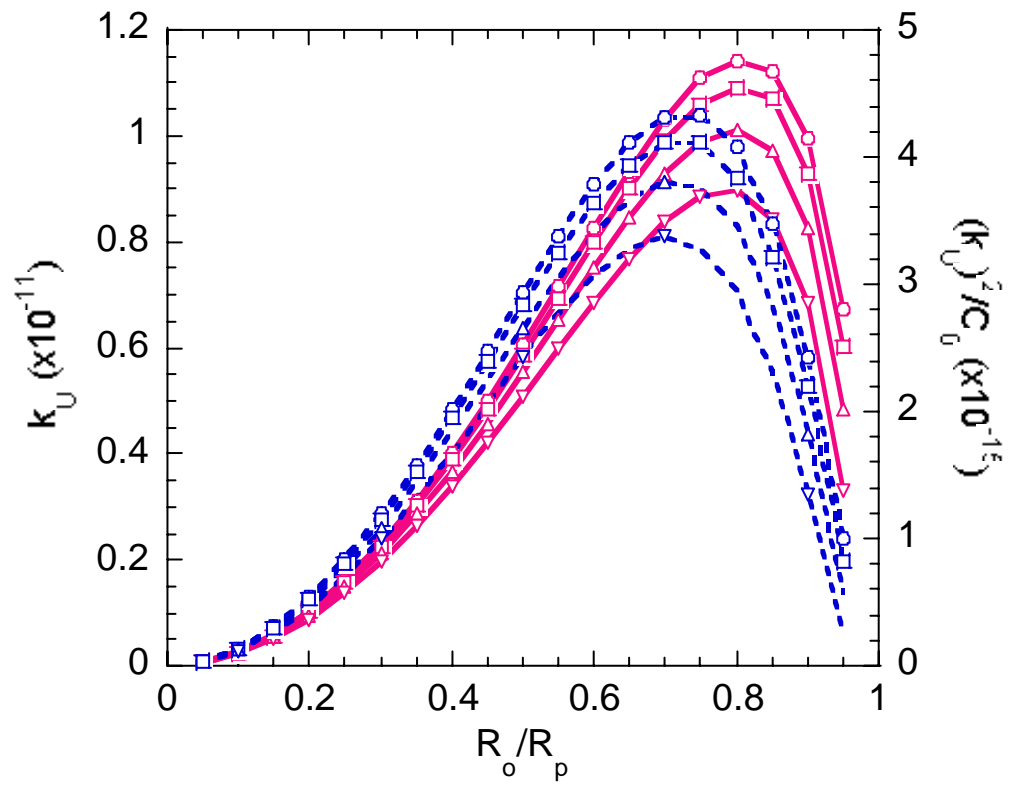

Figure 48 Coefficient $k_{U}$ and $k_{U}^{2} / C_{0}$ at different $\mathrm{PZT}$ /passive plate radius ratio

In Fig. 48, the solid curves represent $k_{U}$ (from top to bottom: $R_{i} / R_{o}=0.1,0.2,0.3$ and 0.4 ) and the dot curves represent $k_{U}^{2} / C_{0}$ (from top to bottom: $R_{i} / R_{o}=0.1,0.2,0.3$ and 0.4 ). So it is obvious that increasing the inner/outer radius ratio of the PZT ring will reduce both $k_{U}$ and 
$k_{U}^{2} / C_{0}$. Increasing $R_{i}$ will reduce the capacitance but will also reduce the deflection at the same time. As a whole, $k_{U}^{2} / C_{0}$ is decreasing when $R_{i}$ is increasing. Also can be observed is that there exists an optimal PZT/passive plate radius ratio for both $k_{U}$ and $k_{U}^{2} / C_{0}$. The optimal ratio for $k_{U}$ (about 0.8) is larger than the optimal ratio for $k_{U}^{2} / C_{0}$ (about 0.7 ) because a larger PZT ring radius results in a larger capacitance.

Keeping both the inner/outer radius ratio of the PZT ring and the PZT/passive plate radius ratio fixed, the curves of both $k_{U}$ and $k_{U}^{2} / C_{0}$ as a function of the passive plate radius can be obtained. As shown in Fig. 49, increasing the radius of the passive plate will increase both $k_{U}$ and $k_{U}^{2} / C_{0}$.

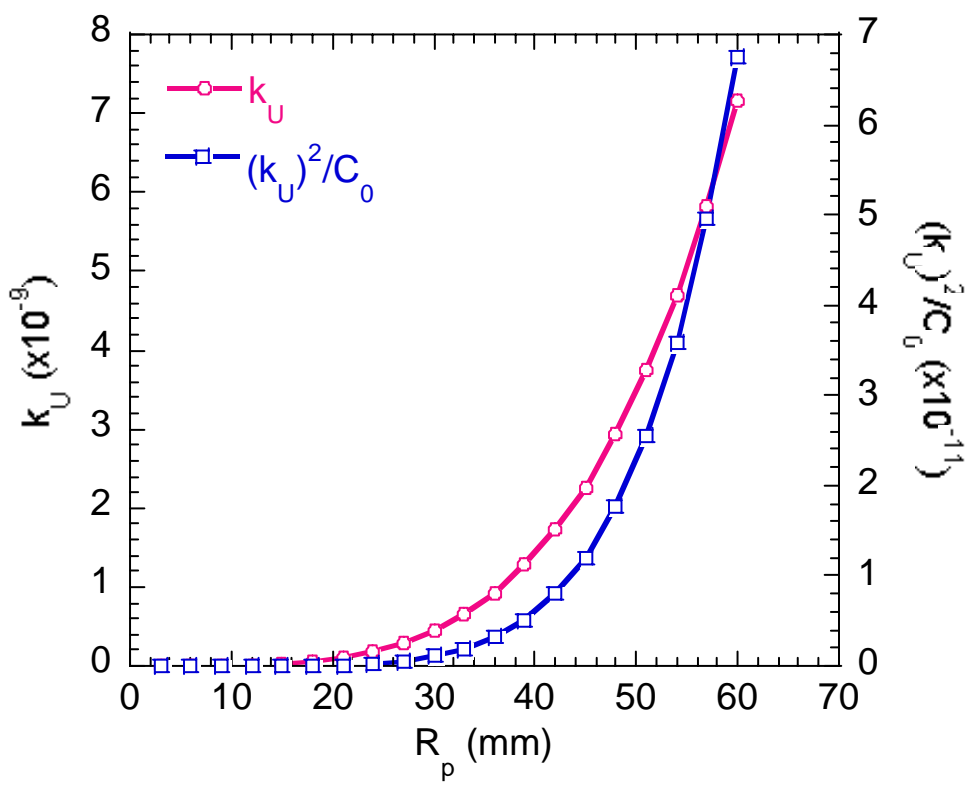

Figure 49 Coefficient $k_{U}$ and $k_{U}^{2} / C_{0}$ at different passive plate radius with fixed PZT/passive plate radius ratio

The effect of the thicknesses on the performance of the pump actuator is depicted in Fig. 50 and Fig. 51. The optimal thickness of the PZT ring exists for both $k_{U}$ and $k_{U}^{2} / C_{0}$. Since a smaller PZT ring thickness leads to a larger capacitance, the value of the optimal PZT ring thickness for $k_{U}^{2} / C_{0}$ (about $0.25 \mathrm{~mm}$ ) is large than the value of the optimal thickness for $k_{U}$ (about $0.1 \mathrm{~mm}$ ). 


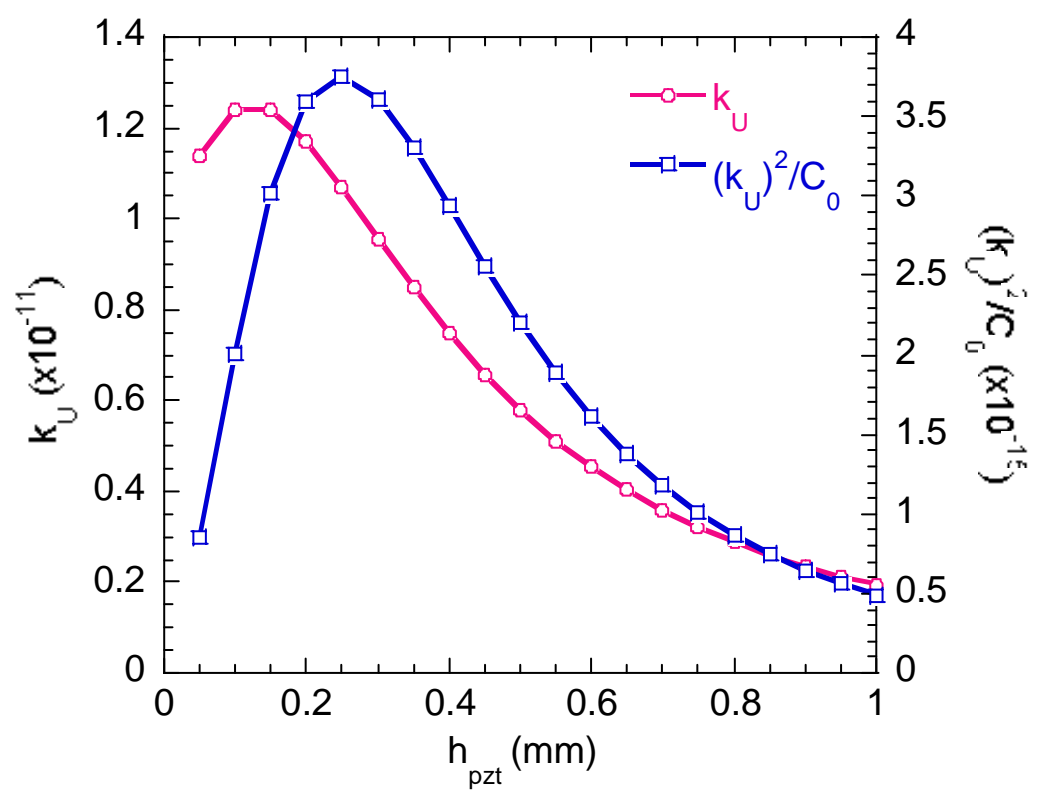

Figure 50 Coefficient $k_{U}$ and $k_{U}^{2} / C_{0}$ at different PZT layer thickness

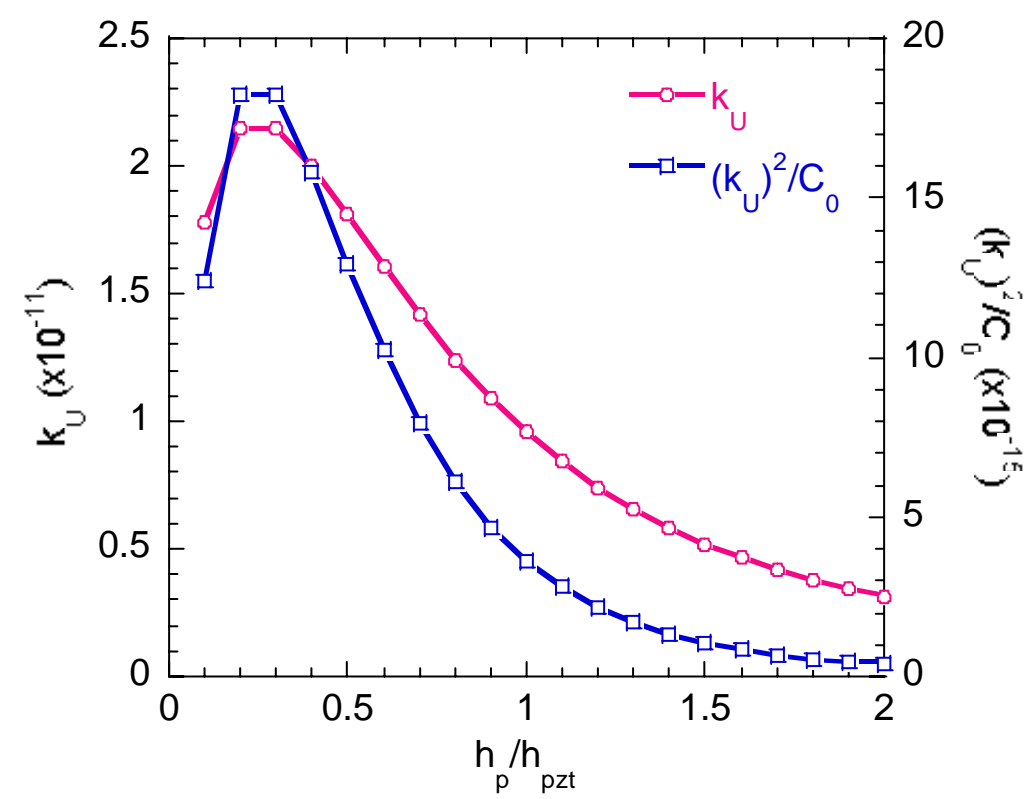

Figure 51 Coefficient $k_{U}$ and $k_{U}^{2} / C_{0}$ at different passive/PZT layer thickness ratio with fixed PZT layer thickness 
By keeping the thickness of the PZT ring fixed, it can also be found that the optimal PZT/passive plate thickness ratio exits for both $k_{U}$ and $k_{U}^{2} / C_{0}$. Since the thickness of the passive plate won't affect the capacitance, the values of both optimal thickness ratios are the same and about 0.3 in this case.

\subsection{SUMMARY}

The theoretical models of both the disk-type bending actuator and the ring-type bending actuator are compared with the numerical and experimental models. Although certain differences exist between the analytical solutions and the results obtained by other methods, it is still possible to acquire a fair estimation of the pump performance using the theoretical models. The effects of material properties and dimensions on the flow rate and power consumption of the micropump are investigated theoretically. The following principles for the design of the micropump can be summarized:

1. For the micropump using either disk-type bending actuator or ring-type bending actuator, increasing the Young's modulus of the PZT disk or decreasing the Young's modulus of the passive plate will be favorable for both flow rate and power consumption. This means the flow rate will be higher and the power consumption will be smaller. However the relationships between the material properties and the performance of the micropump are not linear. Once the material properties exceed certain limit, the influence on the pump performance will become insignificant.

2. Although the electrical field strength is larger when the thickness of the PZT disk is smaller, the pump performance using a thinner PZT layer is not always better. For both disk-type and 
ring-type, there exists an optimal PZT layer thickness at which the power consumption is the smallest. As for the flow rate, an optimal PZT layer thickness exists for the ring-type but not for the disk-type. The ring-type optimal PZT layer thickness for the flow rate is smaller than that for the power consumption. As for the disk-type, theoretically the smaller the PZT layer thickness, the larger the flow rate. But note that the existence of the maximum allowable electrical field strength will impose a limit on the thickness of the PZT layer.

3. If the thickness of the PZT layer is fixed, an optimal passive/PZY layer thickness ratio can be found for both disk-type and ring-type. Also for both flow rate and power consumption, this optimal value of each type is the same because the capacitance doesn't vary with the thickness of the passive plate.

4. In the disk-type case, if the radius of the passive plate is fixed, an optimal PZT/passive plate radius ratio exists for both flow rate and power consumption. Since larger PZT disk radius results in large capacitance, this optimal radius ratio for the power consumption is smaller than that for the flow rate. If the PZT/passive plate radius ratio is fixed, increasing the passive plate radius will increase the flow rate and reduce the power consumption.

5. In the ring-type case, by keeping both passive plate radius and PZT ring outer radius fixed, it can be found that a smaller PZT ring inner radius is preferred for both flow rate and power consumption. If the inner/outer PZT ring radius ratio is fixed, an optimal PZT/passive plate radius ratio can be observed. This optimal radius ratio for the power consumption is also smaller than that for the flow rate. If all the radius ratios are fixed, increasing the passive plate radius will increase the flow rate and reduce the power consumption. 


\subsection{EXPERIMENTAL CHARACTERIZATION OF MICROPUMP}

\subsection{FABRICATION OF MICROPUMP PROTOTYPE}
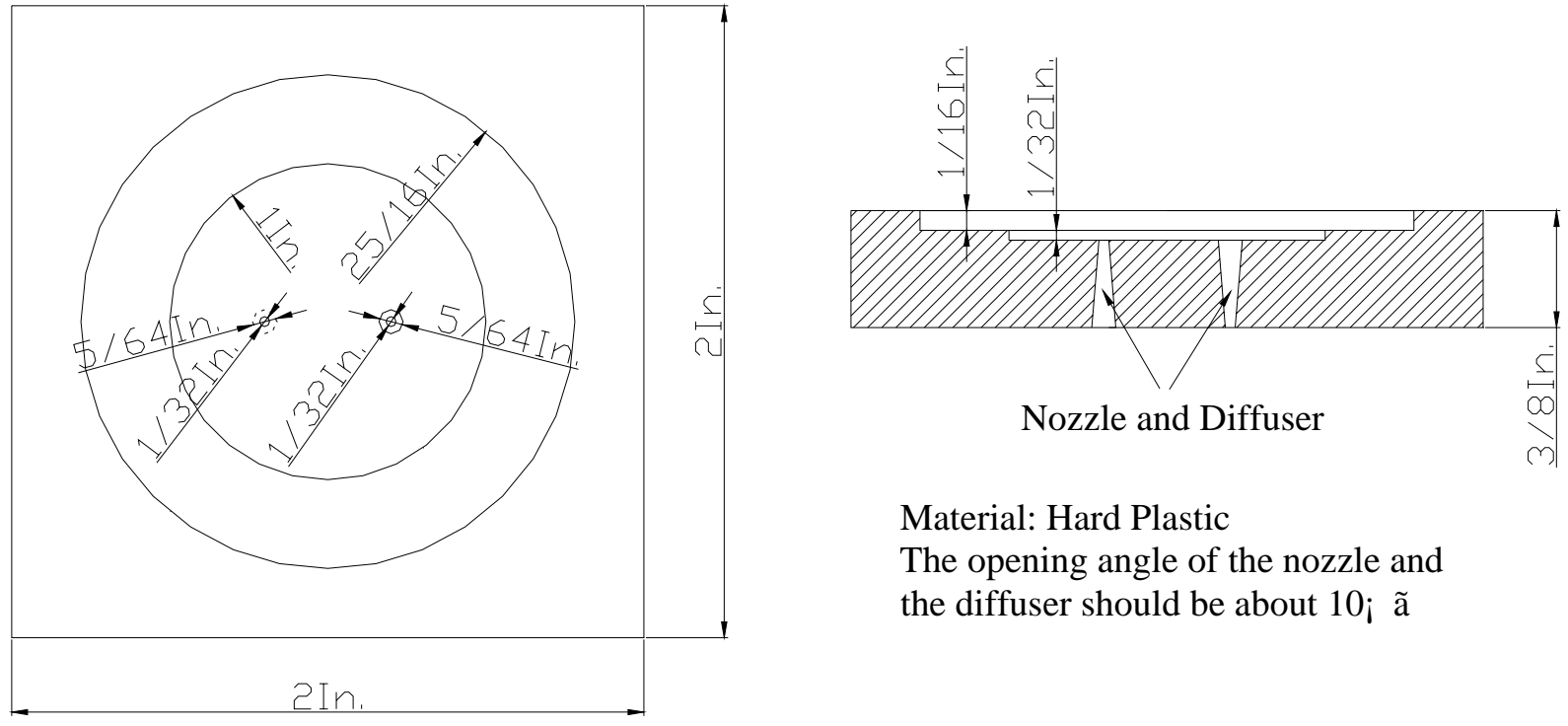

Nozzle and Diffuser

Material: Hard Plastic

The opening angle of the nozzle and the diffuser should be about $10 \mathrm{i}$ ã

Figure 52 Design graph of the micropump housing

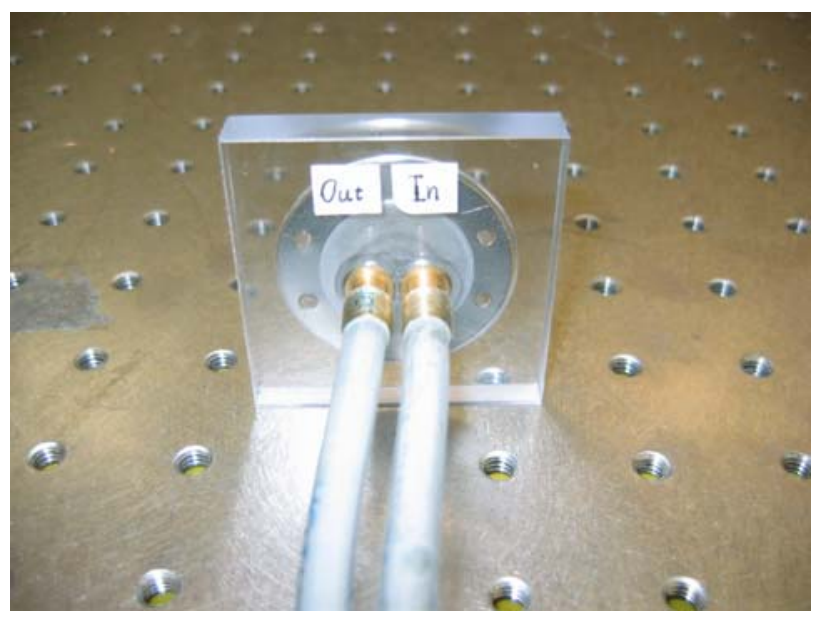

Figure 53 Photo of the piezoelectric valveless micropump prototype 
Based on the previous analysis, the micropump prototype is fabricated in our lab. The material properties and dimensions of this prototype are listed in Table 5. Not all of the design parameters of the prototype are optimal due to the limitations of the experimental conditions and the available materials. To fabricate the thin PZT ring, first a PZT ring with a thickness of $0.8 \mathrm{~mm}$ is cut from a thicker PZT ring using the dicing saw, and then this PZT ring is grinded to the specific thickness. After that, the top and bottom surfaces of the PZT ring are coated with thin gold electrode layer using a sputter coater device. The thin stainless steel disk is bought from McMaster-Carr Supply Company. The PZT ring is bonded onto the stainless steel disk using epoxy. Two very thin bare copper wires are connected to the two electrodes of this ring-type bonding actuator using conductive epoxy. The pump housing is made of hard transparent plastic material by machine shop. By bonding the ring-type bending actuator onto the pump housing with epoxy, the valveless piezoelectric micropump is finally obtained. Fig. 52 shows the top and cross section of the pump housing. Fig. 53 is the photo of this micropump prototype.

\subsection{EXPERIMENTAL SETUP}

The experimental setup is illustrated in Fig. 54. First, a voltage signal (DC or sinusoidal) is generated from a function generator (Stanford Research Systems Model DS345) and enlarged by passing through a power amplifier (AVC Instrumentation 790 Series). Then the enlarged voltage signal is applied to the micropump actuator. A multimeter (FLUKE 45 Dual Display Multimeter) is used to monitor the applied voltage signal. The deflection of the micropump diaphragm is detected by a fiber optical detection system (MTI 2000 Fotonic Sensor). Another device that is not shown in the figure is an oscilloscope (Tektronix TDS 3032), which can measure the original 
voltage signal from the function generator and the response signal from the fiber optical sensor. To measure the water flow rate of this micropump, two water containers are connected to the inlet and outlet of the micropump separately. By measuring the movement the water surface inside the containers with respect to time, the average flow rate and the corresponding pressure head can be obtained. In addition, the impedance and the frequency response of the micropump can be measured by connecting the two electrodes of the micropump actuator to an impedance analyzer (Agilent 4294A or Gamry Electrochemical Measurement System).

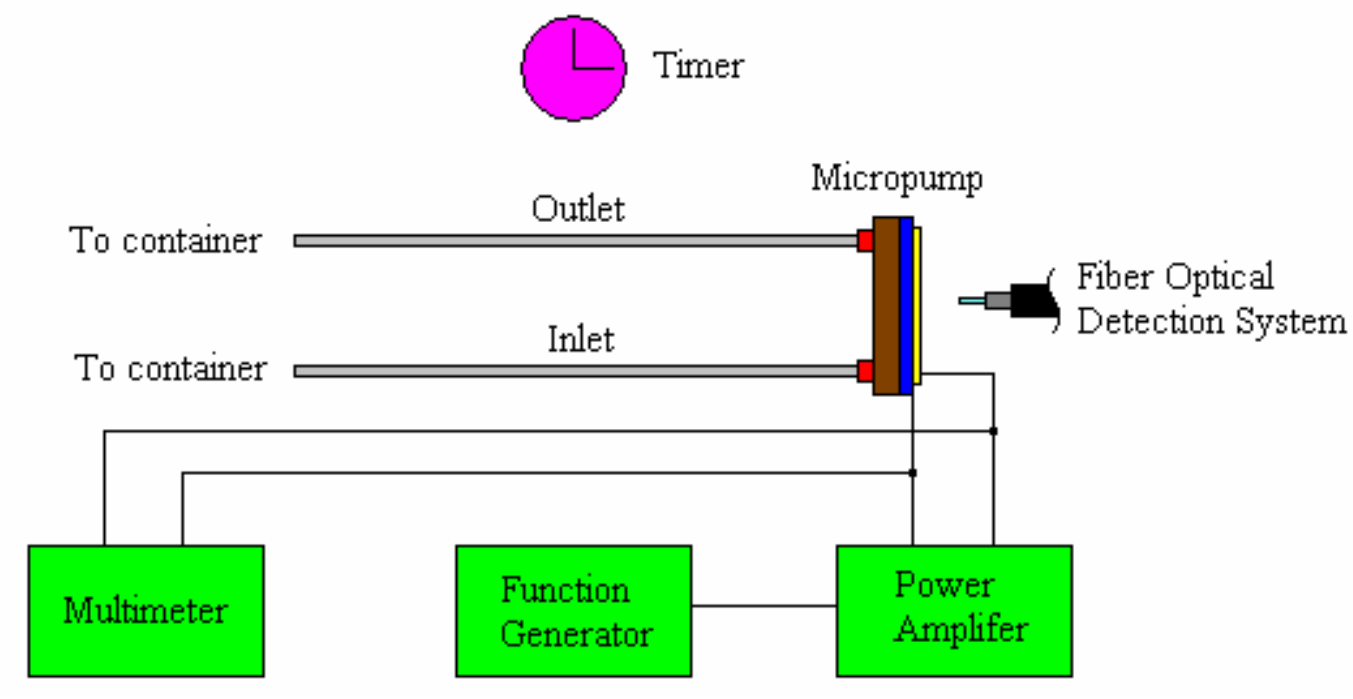

Figure 54 Experimental setup for the micropump measurement

\subsection{RESULTS WITHOUT LIQUID LOADING}

The deflection and the frequency response of the micropump prototype are first measured without liquid loading. As shown in Fig. 55, the relationship between the center deflection of the prototype (without liquid loading) and the applied DC voltage is almost linear. This linear relationship is also predicted by the theoretical analysis although certain difference exits between 
the values of the experimental results and that of the theoretical results. Basically, increasing the applied voltage will increasing the applied electrical field, therefore the deflection and the volume displacement will increase as well. However, the applied electrical field cannot exceed a certain limit or the PZT material will be depolarized. This limit of the PZT-5H used in our prototype is about $8 \mathrm{kV} / \mathrm{cm}$ and for a PZT ring with a thickness of $0.3 \mathrm{~mm}$ the maximum applied voltage should be around $240 \mathrm{~V}$. Higher driving voltage also brings larger power consumption. Fig. 56 shows the same linear relationship between the center deflection and the applied AC voltage. The applied AC voltage is sinusoidal and the frequency is $100 \mathrm{~Hz}$. Note that the $\mathrm{x}$ coordinate is the RMS (root mean square) value so the corresponding deflection is much larger.

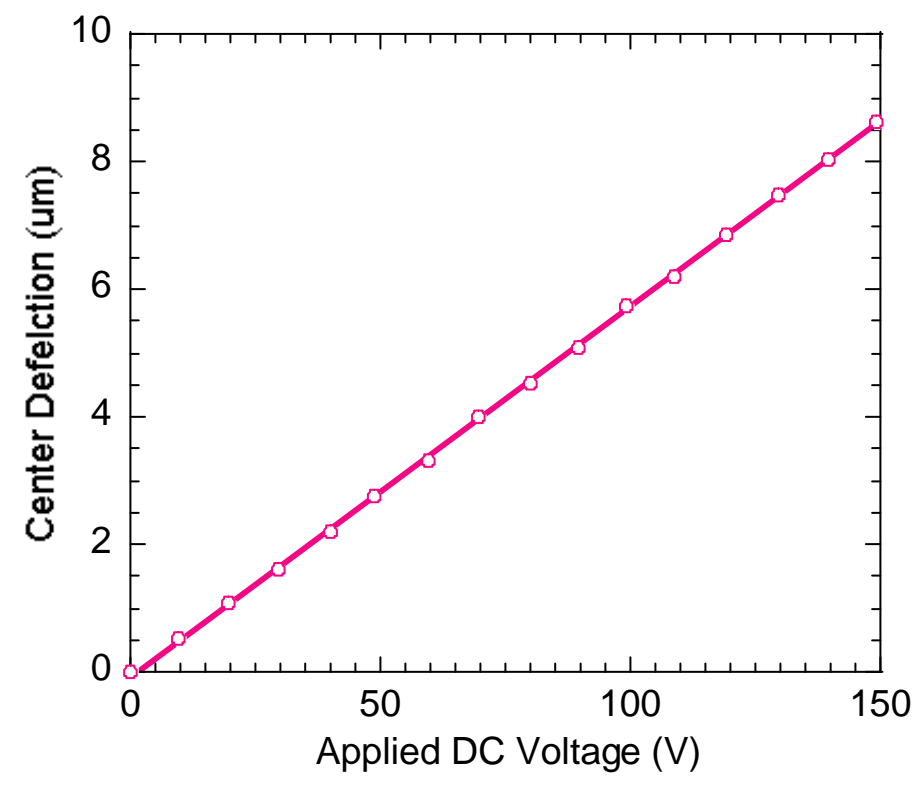

Figure 55 Measured center deflection of the micropump diaphragm under different applied DC voltage (without liquid loading) 


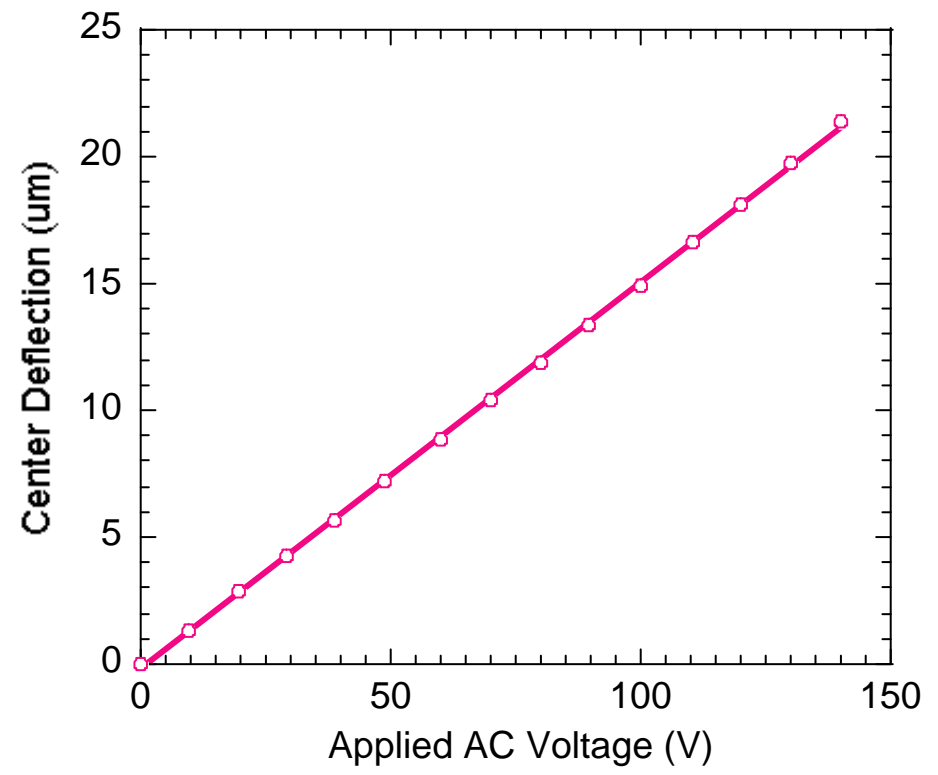

Figure 56 Measured center deflection of the micropump diaphragm under different applied AC voltage (without liquid loading)

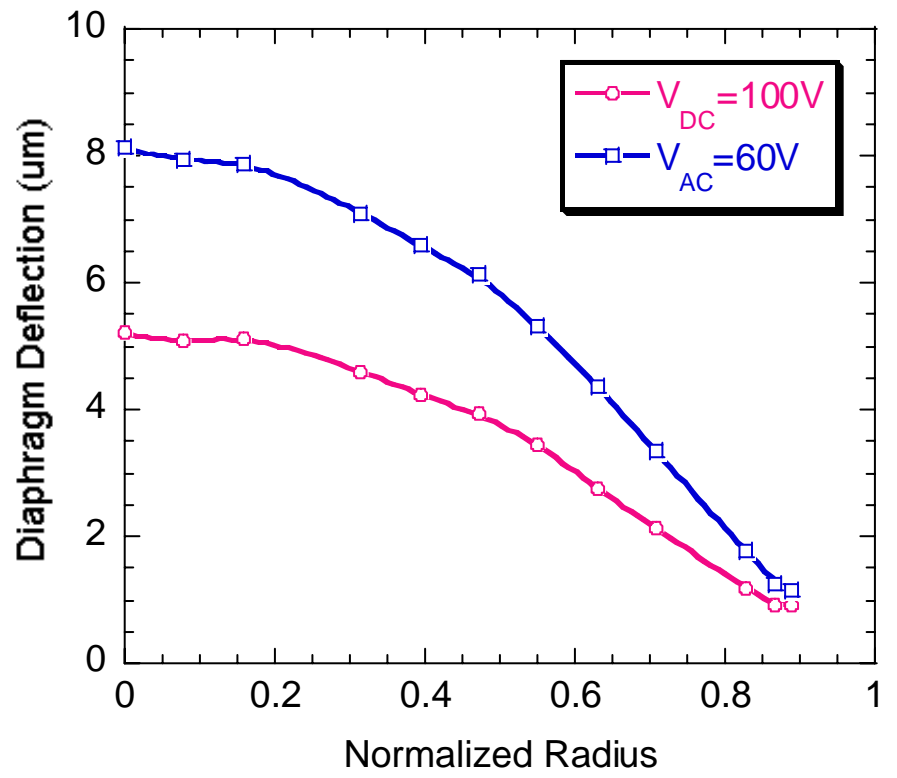

Figure 57 Measured deflection distribution of the micropump diaphragm under applied DC and AC voltage (without liquid loading)

The deflection distribution along the radius is shown in Fig. 57. Both DC and AC voltage gives the similar curve. The maximum deflection appears around the center then decreases when radius increases. Since the bonding process is not perfect, the deflection measured does not reach zero at the outer edge. The comparison of the deflection distribution curves obtained by different 
methods is shown earlier in Fig. 45. In general, all the deflection curves show the similar variation trend and quantitatively the theoretical result is closer to the experimental result.

The other important factor that can influence the performance of the pump is the driving frequency. For the case without liquid loading, two methods are used to measure the frequency response of the pump actuator: One is to measure the center deflection under different driving frequency and the other is to measure the impedance spectroscopy of this actuator. For comparison, the numerical results are also obtained by using the commercial FEM package, ANSYS 6.1. As shown in Fig. 58, the first resonant frequency appears at several $\mathrm{kHz}$ where the deflection reaches the maximum value. Both resonant frequencies obtained by experimental methods are very close while the resonant frequency obtained by ANSYS is much larger.

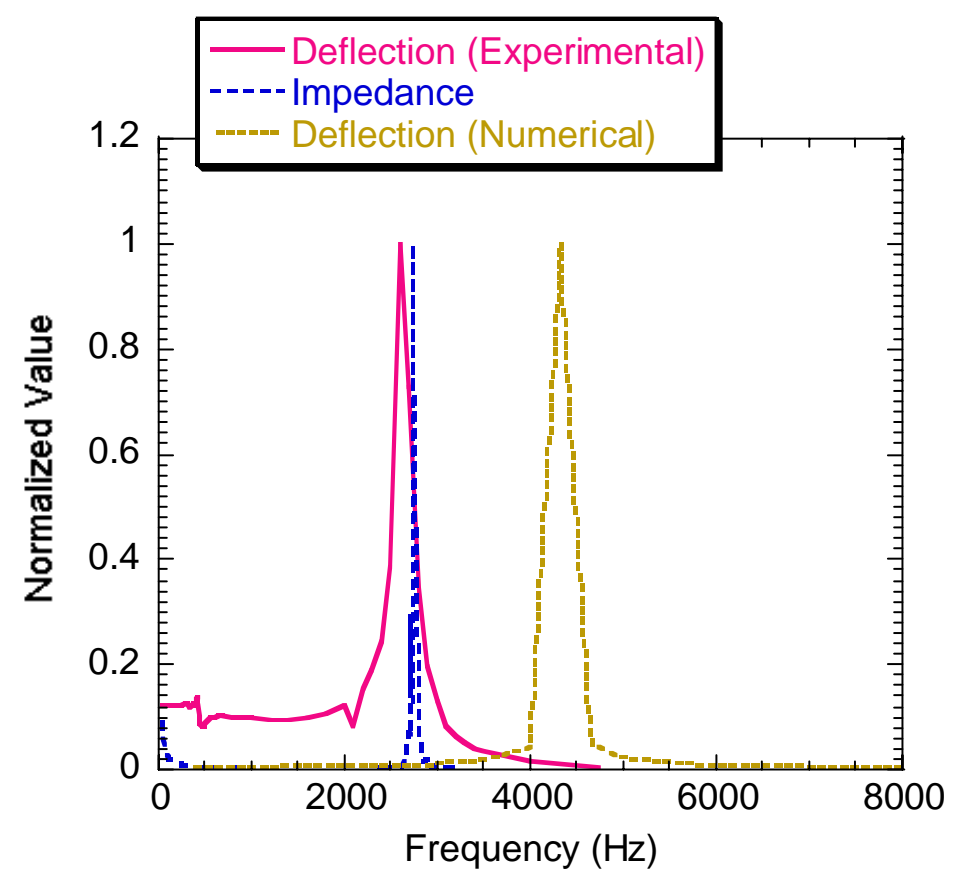

Figure 58 Frequency response of the micropump obtained by different methods (without liquid loading) 


\subsection{RESULTS WITH LUQUID LOADING}

Since the micropump is specifically designed for driving methanol water solution as fuel for the DMFC power system, it is very important to investigate the pumping performance in the operation with liquid loading. Here water is used as working fluid whose property is quite close

to that of the methanol water solution. Concerning the self-priming capability and bubble tolerance, there is a design criterion for the minimum compression ratio of a micropump [72]. During the fabrication of the micropump prototype, the thickness of the pump chamber is made as small as possible but the compression ratio is still much smaller than the theoretical criterion. To solve this problem, a mini pump is used to fill the micropump with water and it turns out that the gas inside the pump chamber can be driven out quite easily. The details about this mini pump will be introduced later.

As shown in Fig. 59, for the case with water loading, the relationship between the center deflection and the applied voltage is also linear. In this measurement, the applied voltage is sinusoidal and the driving frequency is $200 \mathrm{~Hz}$. The frequency responses of the micropump with water loading are also evaluated by both center deflection and impedance spectroscopy. From Fig. 60, it is found that the results obtained by both methods are very close and the resonance frequency is about $200 \mathrm{~Hz}$, which is far less than the case without water loading. 


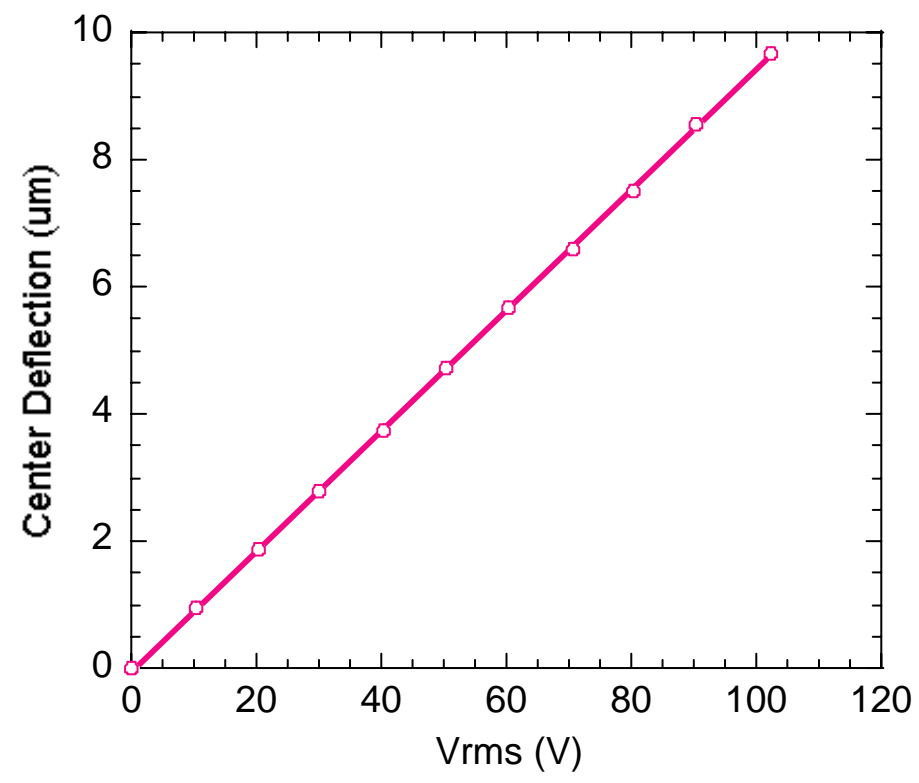

Figure 59 Measured center deflection of the micropump diaphragm under different applied AC voltage (with liquid loading)

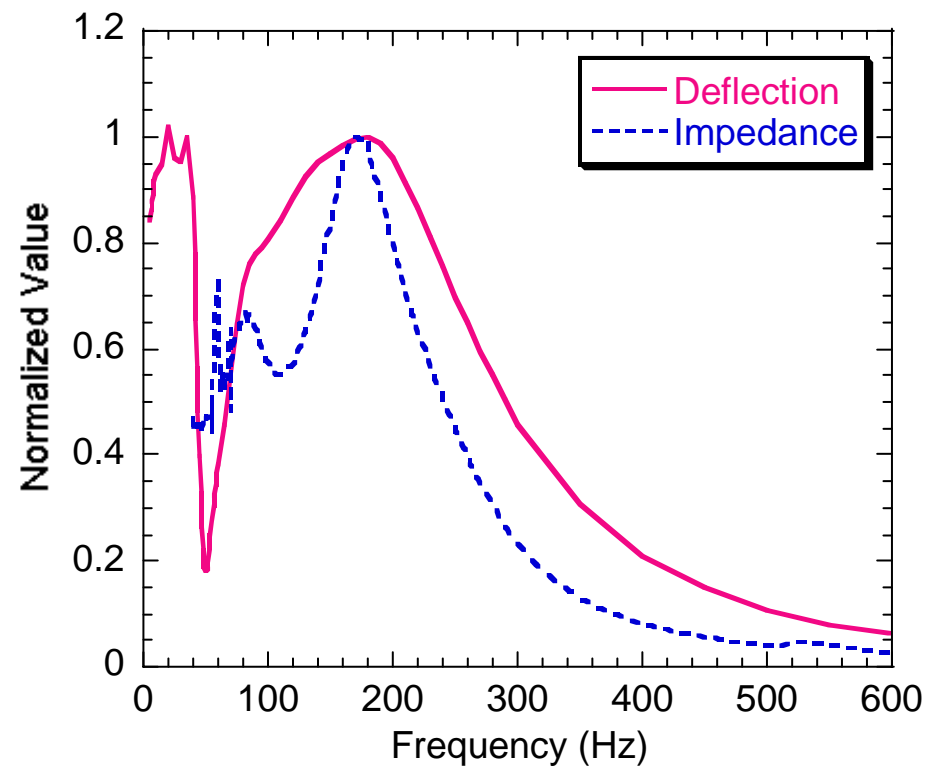

Figure 60 Frequency response of the micropump obtained by different methods (with liquid loading) 


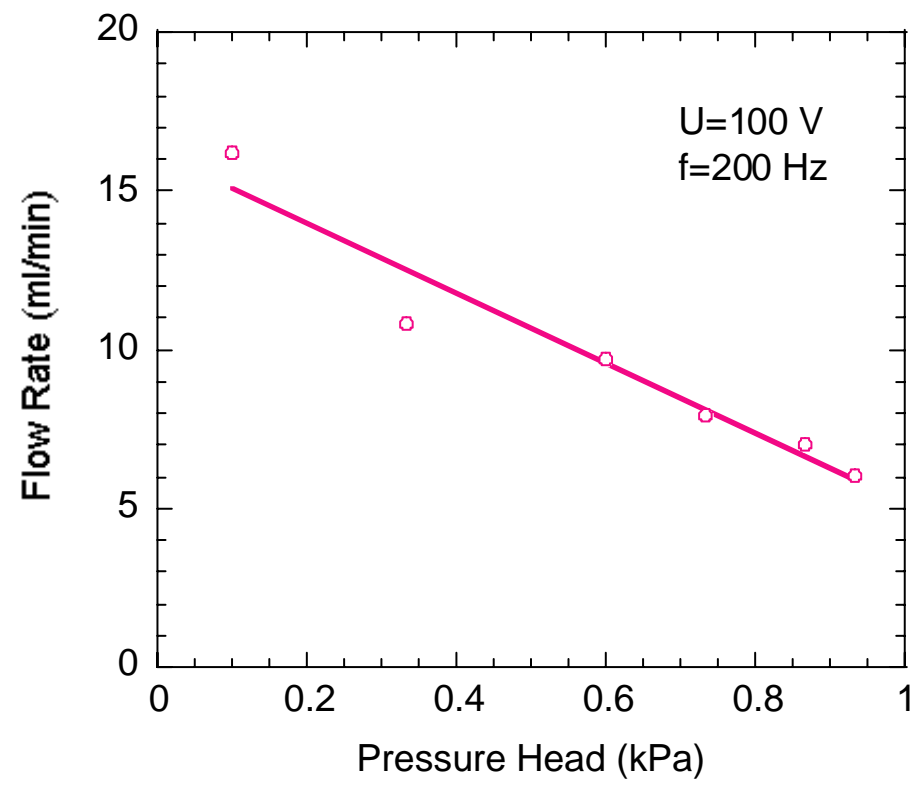

Figure 61 Measured flow rate versus pressure head (water as working fluid)

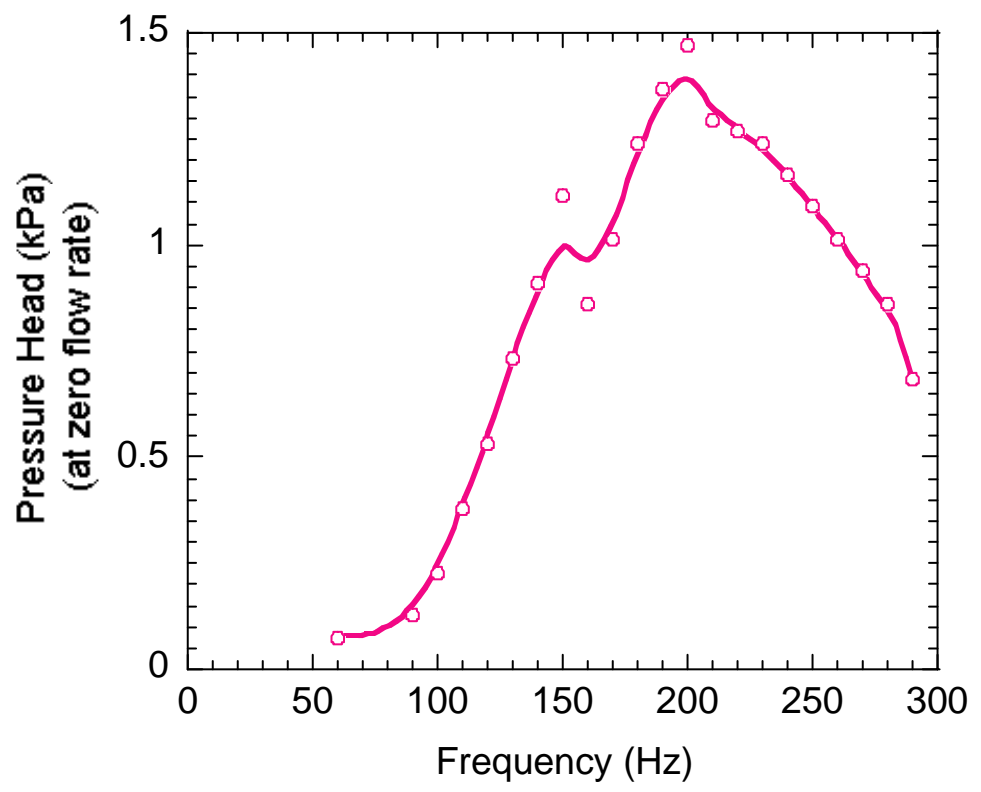

Figure 62 Measured pressure head at zero flow rate versus driving frequency (Vrms=100V)

The results of the water flow rate measured under different pressure head are shown in Fig. 61. The larger flow rate corresponds to the lower pressure head. Using this micropump, a flow rate of $5 \mathrm{ml} / \mathrm{min}$ at $1 \mathrm{kPa}$ can be delivered when applying a $100 \mathrm{~V}$ voltage with a driving frequency of $200 \mathrm{~Hz}$. The maximum value of the measured capacitance is about $8.22 \mathrm{nF}$ and it can be 
estimated that the maximum power consumption should be around $50 \mathrm{~mW}$. The pressure head of the micropump at zero volume flow rate is measured when the actuation frequency is changing. Shown in Fig. 62, the similar resonance frequency is found to be around $200 \mathrm{~Hz}$.

\subsection{SUMMARY}

A valveless piezoelectric micropump prototype has been fabricated and tested in our lab. A linear relationship between the deflection and the applied voltage is found for both the case with liquid loading and the case without liquid loading. This experimental result fits well with the theoretical analysis introduced earlier. The frequency response of the micropump has also been measured and it is observed that at the resonance frequency the deflection reaches the maximum value. The value of the resonance frequency obtained by impedance measurement is very close to that obtained by deflection measurement. The resonance frequency of the case without liquid loading is close to $3 \mathrm{kHz}$ while the resonance frequency of the case with liquid loading is much smaller and around $200 \mathrm{~Hz}$. Since large deflection leads to large flow rate, the best performance can be obtained by driving the micropump at the resonance frequency. Actuated by a $100 \mathrm{~V}$ voltage at $200 \mathrm{~Hz}$, the micropump prototype can supply a $5 \mathrm{ml} / \mathrm{min}$ of water flow rate at $1 \mathrm{kPa}$. With this micropump prototype, the performance of the DMFC device driven by micropump can be investigated. 


\subsection{PERFORMANCE ANALYSIS OF DMFC DRIVEN BY MICROPUMP}

As mentioned earlier, among various types of fuel cells, PEMFC and DMFC are applicable for the portable electronics due to their low operation temperature. In comparison with DMFC, PEMFC device that uses directly hydrogen as fuel has a better performance but suffers from the storage problem of hydrogen. So far the storage and transportation of hydrogen are still not cost effective for commercial applications. And the fuel cell with a reformer to convert the liquid fuel to hydrogen gas is also less favorable because it increases the complexity of the system integration and generates undesired byproduct (carbon monoxide, $\mathrm{CO}$ ) during the reforming process. Therefore DMFC using liquid methanol solution as fuel directly is much more straightforward and attractive.

To optimize a miniaturized DMFC power system, many engineering issues need to be considered, which includes thermal management, water management, methanol crossover, fuel delivery, oxidant supply, and power conditioning. The research here mainly focuses on the fuel delivery. For medium and large size DMFC, conventional pumps can be used to drive the fuel flowing through the fuel cell. For miniaturized DMFC, the flow rate of fuel is very small and there are several options for fuel delivery. Some researchers [54] have presented a pump-less and self-activated design of DMFC stack, in which gravitational and capillary forces were used to feed the liquid methanol solution. By their calculation, the fuel supply rate was adequate for acceptable performance. This design was quite interesting and simple but no experimental results were reported. In addition, the fabrication of the DMFC stack seems to be very complicated. The 
more general way is to choose a micropump. It is reported that peristaltic pumps were used in the experimental study of DMFC [55, 56]. The flow rate of fuel varied from less than $1 \mathrm{ml} / \mathrm{min}$ to 5 $\mathrm{ml} / \mathrm{min}$ and the active cell area ranged from $9 \mathrm{~cm}^{2}$ to $25 \mathrm{~cm}^{2}$. The operation performance of their fuel cell systems was in the acceptable range. Therefore it seems to be more practical and easier to employ a micropump for fuel delivery.

The idea to design a miniaturized DMFC power system driven by piezoelectric valveless micropump has been introduced earlier. This type of micropump is easy to fabricate, easy to control, and the power consumption can be very small for an optimal design. Both theoretical and experimental researches on the performance of this micropump have been presented in the previous chapters. In this chapter, the performance of the whole system combining DMFC with micropump will be discussed.

\subsection{ANALYTICAL ANALYSIS}

To analyze the performance of DMFC with a micropump fuel delivery system, a theoretical model describing the relationship between the flow rate of the liquid fuel and the fuel cell characteristic J-V curve would be more convenient. Here, for simplicity, some appropriate experimental data from literature are chosen to discuss the performance of fuel cells.

Up to now some experimental data have been reported about the performance of DMFC under different operation conditions. For example, a single cell $\left(7.6 \mathrm{~cm}^{2}\right)$ performance was evaluated over the range of $60-120{ }^{\circ} \mathrm{C}$ with methanol concentrations of $0.5-4.0 \mathrm{M}$, flow rates of $9 \mathrm{ml} / \mathrm{min}$, and an oxygen saturation of $105 \mathrm{sccm}$ [57]. A current density of $230 \mathrm{~mA} / \mathrm{cm}^{2}$ at $0.55 \mathrm{~V}$ was obtained from 2.5 M methanol. The DMFC experiments under air operation at low over pressure 
were conducted [58]. A current density of $100 \mathrm{~mA} / \mathrm{cm}^{2}$ and a $0.5 \mathrm{~V}$ voltage at $80{ }^{\circ} \mathrm{C}$ was observed from $0.5 \mathrm{M}$ methanol. The electrode area of their experimental cells was $3 \mathrm{~cm}^{2}$. An optimum methanol concentration of $2 \mathrm{M}$ for a $20 \mathrm{~cm}^{2}$ single cell under $110{ }^{\circ} \mathrm{C}$ was also reported [59]. The current density and the corresponding cell voltage were in the similar range as in [58]. The experiment results presented in [60] indicated that there is a complex relation between the effect of flow velocity and the influence of the production rate of $\mathrm{CO}_{2}$.

The latest experimental data on DMFC can be found in [61]. A series of J-V characteristic curves were measured for a single fuel cell assembly $\left(5 \mathrm{~cm}^{2}\right)$ operating under different operation temperatures (80, 60 and $40{ }^{\circ} \mathrm{C}$ ), different methanol flow rates $(5,0.5$ and $0.15 \mathrm{ml} / \mathrm{min})$, and different methanol concentrations (2, 1 and $0.5 \mathrm{M}$ ). The performance analysis that will be discussed later is mainly based on this group of data. From the data obtained, it is found that at low current density (less than $100 \mathrm{~mA} / \mathrm{cm}^{2}$ ), the influence of changing methanol concentration (from $0.5 \mathrm{M}$ to $2 \mathrm{M}$ ) on the characteristic J-V curve of DMFC is not significant. The same phenomenon is also observed from the experimental data obtained by other researchers. Therefore, in the design presented in this dissertation, initially a $2 \mathrm{M}$ methanol solution can be filled into the chambers. During the operation, the concentration of the methanol solution will decrease due to the fuel consumption. But the performance of the fuel cell won't drop too much if the current density is not very high and the methanol concentration is still larger than $0.5 \mathrm{M}$. A certain range of methanol concentration can be maintained through continuous fuel flow from the fuel supply manifold.

Fig. 63 shows the experimental data of a single fuel cell running at $40{ }^{\circ} \mathrm{C}$. The concentration of methanol solution is $1 \mathrm{M}$. This specific data are chosen for calculation because the operation temperature is very close to that of the commercial applications and the concentration is in the 
middle region. Based on Fig. 63, the power density can be calculated as shown in Fig. 64. The maximum value is reached when the current density is around $120 \mathrm{~mA} / \mathrm{cm}^{2}$ and the cell voltage is around $0.35 \mathrm{~V}$. If our design is set to be running under this condition, for a $1 \mathrm{~W}$ fuel cell assembly, the active area needed is then $25 \mathrm{~cm}^{2}(5 \mathrm{~cm}$ by $5 \mathrm{~cm})$ and the total current is about 3

A.

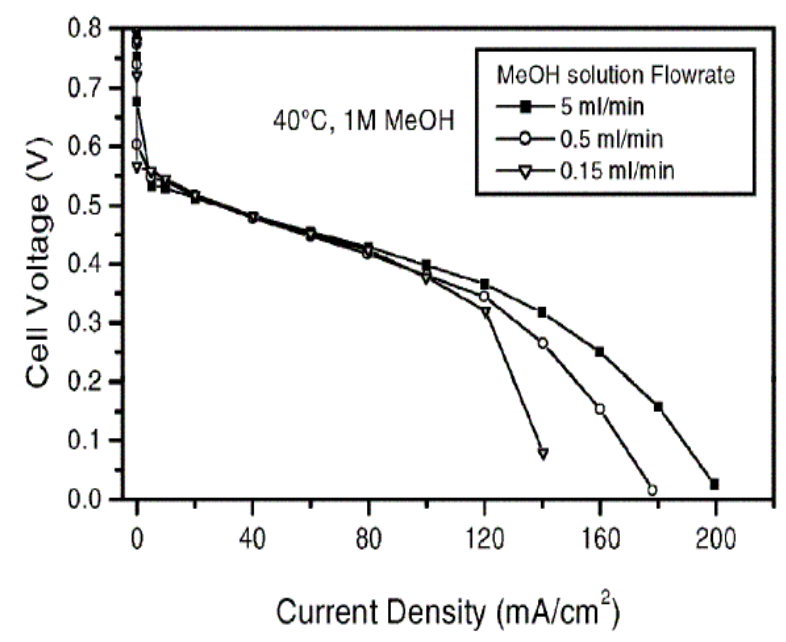

Figure 63 Selected experimental $\mathrm{J}-\mathrm{V}$ data of a single fuel cell at different fuel flow rate [61]

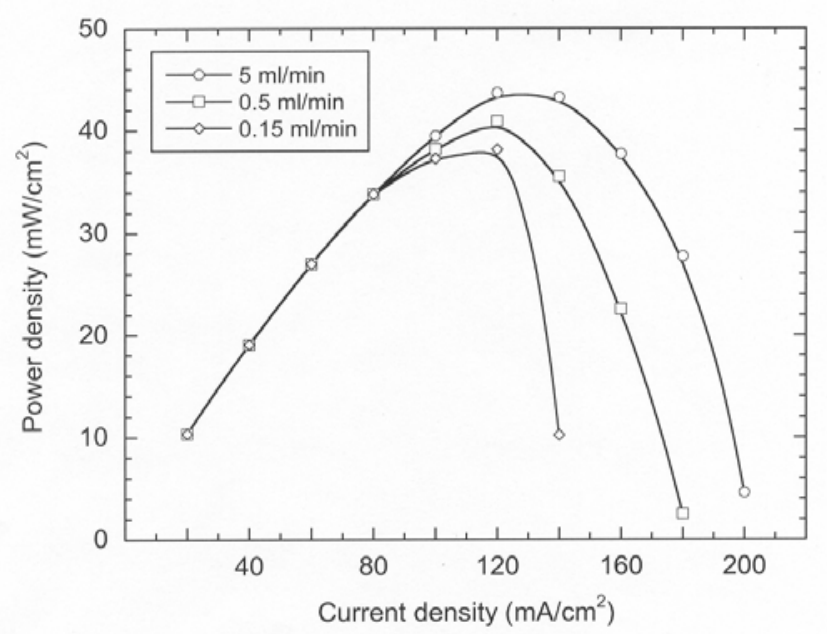

Figure 64 Calculated power density of a single fuel cell at different fuel flow rate 
The next thing we need to know is the least fuel flow rate required under this operation condition. As well known, the electrochemical reactions of a DMFC are:

$$
\begin{aligned}
& \text { Anode Reaction: } \mathrm{CH}_{3} \mathrm{OH}+\mathrm{H}_{2} \mathrm{O} \rightarrow \mathrm{CO}_{2}+6 \mathrm{H}^{+}+6 e^{-} \\
& \text {Cathode Reaction: } \frac{3}{2} \mathrm{O}_{2}+6 \mathrm{H}^{+}+6 e^{-} \rightarrow 3 \mathrm{H}_{2} \mathrm{O} \\
& \text { Overall Reaction: } \mathrm{CH}_{3} \mathrm{OH}+\frac{3}{2} \mathrm{O}_{2} \rightarrow \mathrm{CO}_{2}+2 \mathrm{H}_{2} \mathrm{O}
\end{aligned}
$$

As shown in the above equations, ideally 1 mol Methanol will react with $1 \mathrm{~mol}$ of water and generate 6 mols of electrons. Therefore, the relation between the total generated current and the flow rate of consumed methanol solution can be described as

$$
I=Q \times D_{M} \times 6 \times F
$$

Here, $D_{M}$ is the mol density of the methanol solution and $F$ is Faraday's constant (96485 C/mol). Based on Eq. (6.4), for $1 \mathrm{M}$ methanol, the least fuel flow rate required is approximately $0.31 \mathrm{ml} / \mathrm{min}$.

The material properties and the dimensions of the micropump actuator used in our design are listed in Table 5. As mentioned before, there are two different designs of nozzle/diffuser elements that can be used to direct the flow. For the first design, the small opening angles are small (normally less than $20^{\circ}$ ) and the diffuser direction is the positive flow direction. For the second design, the opening angle is $70.5^{\circ}$ and the positive flow direction is in the converging wall direction. In the theoretical analysis, a conical nozzle element with sharp inlet and outlet is chosen because the performance is not the best and the estimation should be safe. The opening angle of this nozzle is $70.5^{\circ}$. The minimum diameter is $1 \mathrm{~mm}$ and the maximum diameter is 3.15 $\mathrm{mm}$. By following the same analysis method presented in [33], $\xi_{\text {positive }}$ and $\xi_{\text {negative }}$ of this nozzle 
are found to be 1.005 and 1.46 respectively. The ratio of the pressure loss coefficients, $\eta$ is then about 1.45. Since the dimensions and materials of the pump actuator and nozzle/diffuser elements have been determined, the actuation voltage needed to meet the specific flow rate requirement can be calculated by Eq. (3.73). For example, given the actuation frequency is 100 $\mathrm{Hz}$ and the fuel flow rate is $1 \mathrm{ml} / \mathrm{min}, U_{m}$ is then equal to $71 \mathrm{~V}$. By using Eq. (3.83), the maximum electrical power consumption is about $70 \mathrm{~mW}$, which is $7 \%$ of the total power produced by fuel cell. Comparing with a similar micropump [34] that can provide a water flow rate up to $1 \mathrm{ml} / \mathrm{min}$ while consume approximately $50 \mathrm{~mW}$ power, this estimation is not far away from the real case. Furthermore, some methods that will be discussed later can be applied to decrease the power consumption of the micropump.

Fig. 65 shows the net output power by subtracting the power consumed by the piezoelectric pump from the total power generated by fuel cell under different operation conditions at a driving frequency of $100 \mathrm{~Hz}$. Data from Fig. 63, which was obtained from the experiments on a 5 $\mathrm{cm}^{2}$ fuel cell, are used for calculations. So the results may not be precise but the trend should be similar. The negative output power means that the power generated by fuel cell is not enough to drive the micropump. It is obvious that the fuel flow rate of $0.5 \mathrm{ml} / \mathrm{min}$ is preferred.

From Eq. (3.83), it can be found that increasing the driving frequency while maintaining the same flow rate can reduce the electrical power consumption of the piezoelectric micropump. Fig. 66 shows the output power curves at a driving frequency of $200 \mathrm{~Hz}$. The fuel cell performance at the fuel flow rate of $5 \mathrm{ml} / \mathrm{min}$ is improved but the net power output is still too small. The other way to achieve a high flow rate with a reasonable net power output is using two micropumps connected in parallel. 
The performance of two micropumps with a flow rate of $2.5 \mathrm{ml} / \mathrm{min}$ each is also shown in Fig. 65 and Fig. 66, which is much better than that of one micropump with a flow rate of $5 \mathrm{ml} / \mathrm{min}$. However the tradeoff is that the material cost and the system complexity are increased.

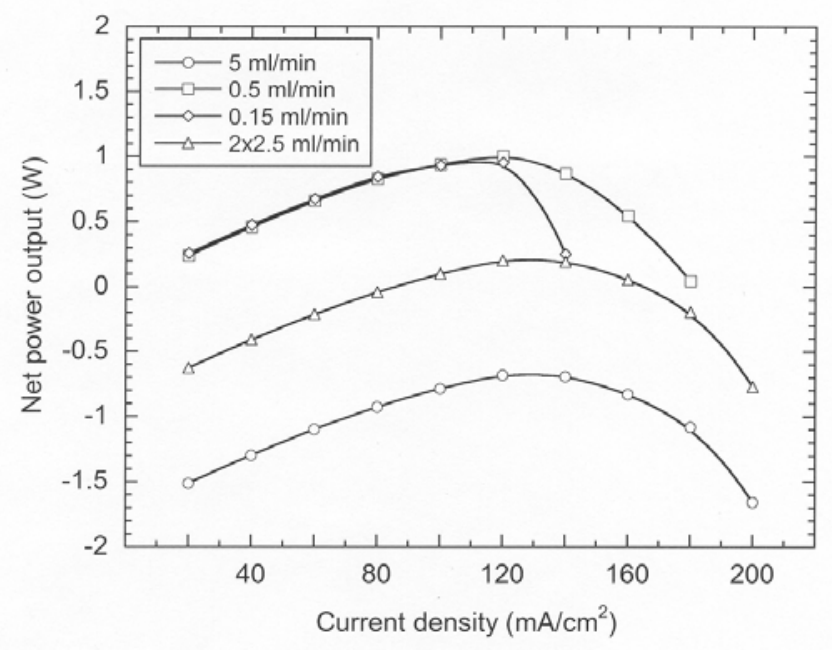

Figure 65 Net power output under different operation conditions (Actuation frequency $=100 \mathrm{~Hz}$ )

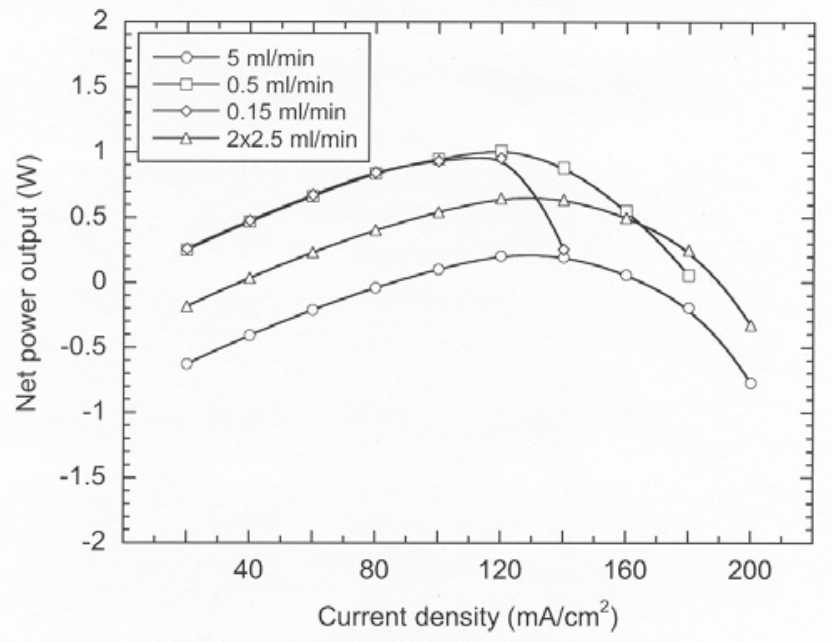

Figure 66 Net power output under different operation conditions (Actuation frequency $=\mathbf{2 0 0 H z}$ )

When the driving frequency is equal to the resonance frequency, the displacement of the diaphragm will reach the maximum value. After that, to increase the driving frequency further won't improve the pump performance. Also it should be noted that during the high frequency range (close to the resonance frequency), our quasi-static model may not be applicable and a 
dynamic model need to be established. Other than a higher driving frequency, larger $d_{31}$ and $\eta$ values are also preferred. Smaller dielectric permittivity $\varepsilon_{33}^{T}$ of piezoelectric material corresponds to smaller power consumption. The effects of dimensions of the micropump actuator have already been discussed in earlier chapter.

\subsection{EXPERIMENTAL ANALYSIS}

Due to the limitation of the resources and time available, a prototype of the miniaturized DMFC power system integrated with micropump has not been finished yet. But separate DMFC device and micropump prototype are available and some useful experimental data can be collected.

Fig. 67 is a photo of the DMFC device tested in our lab. This fuel cell is made of a Nafion ${ }^{\circledR}$ membrane layer sandwiched by two electrode layers with catalysts deposited on them. The methanol water solution is pumped into the anode side while the cathode side is exposed to air through meshed plate. The dimension of this device is about $80 \times 80 \times 20(\mathrm{~mm})$. The photo of the micropump prototype fabricated in our lab is shown earlier in Fig. 53. The pump actuator is made of two layers bonding together: one is a circular stainless steel plate; the other is a PZT-5H ring. The dimension of the micropump is about $50 \times 50 \times 9(\mathrm{~mm})$. Besides the micropump prototype, a peristaltic mini pump has been used to drive fuel into the DMFC device. And it has also been applied to fill the micropump prototype with liquid. The flow rate of this mini pump is variable between 0.03 and $8.2 \mathrm{ml} / \mathrm{min}$. The dimension of it is $168 \times 120 \times 115(\mathrm{~mm})$. The

average power consumption of this pump is about several watts. Its photo is shown in Fig. 68. A KEITHLEY 239 High Current Source Measure Unit is used to measure the characteristic curve of the DMFC device. 


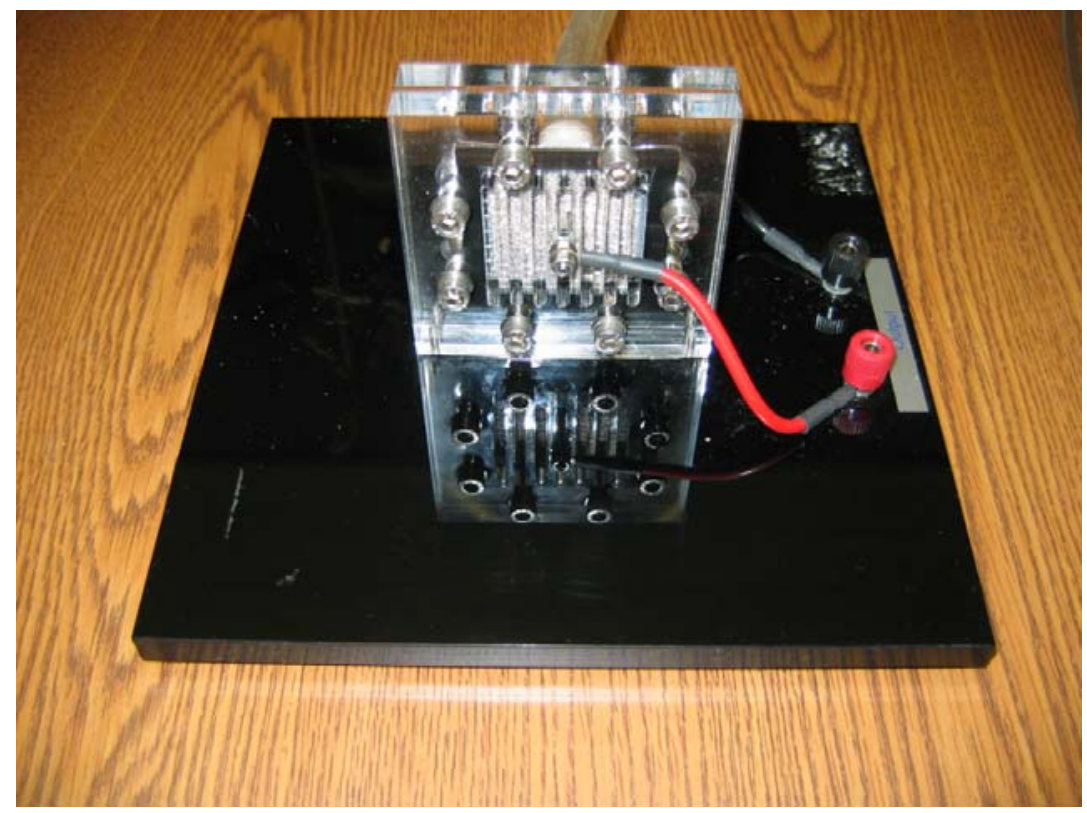

Figure 67 Photo of the DMFC device used in the experiments

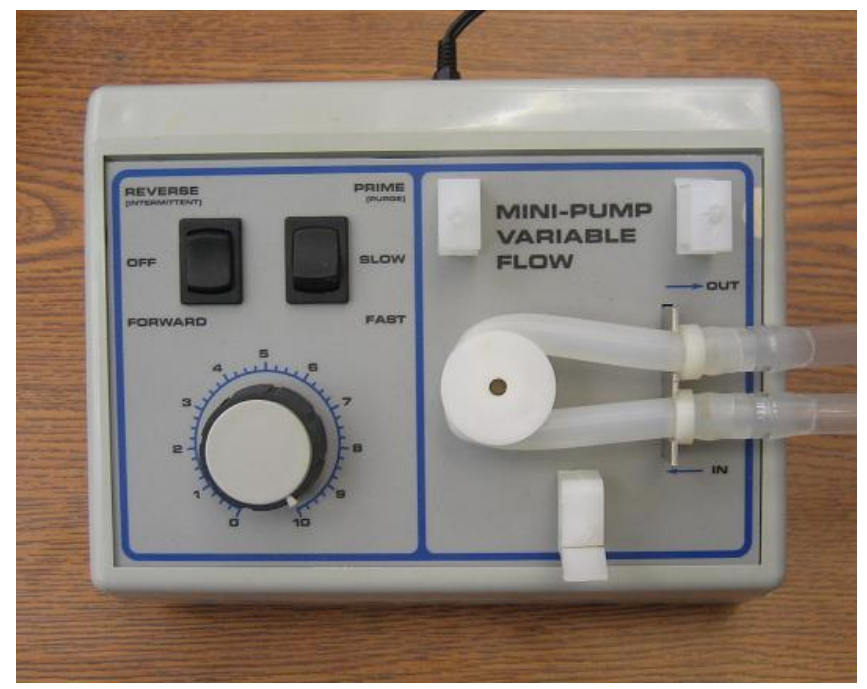

Figure 68 Photo of the peristaltic variable flow mini pump 


\subsubsection{Performance of the DMFC device without pump}

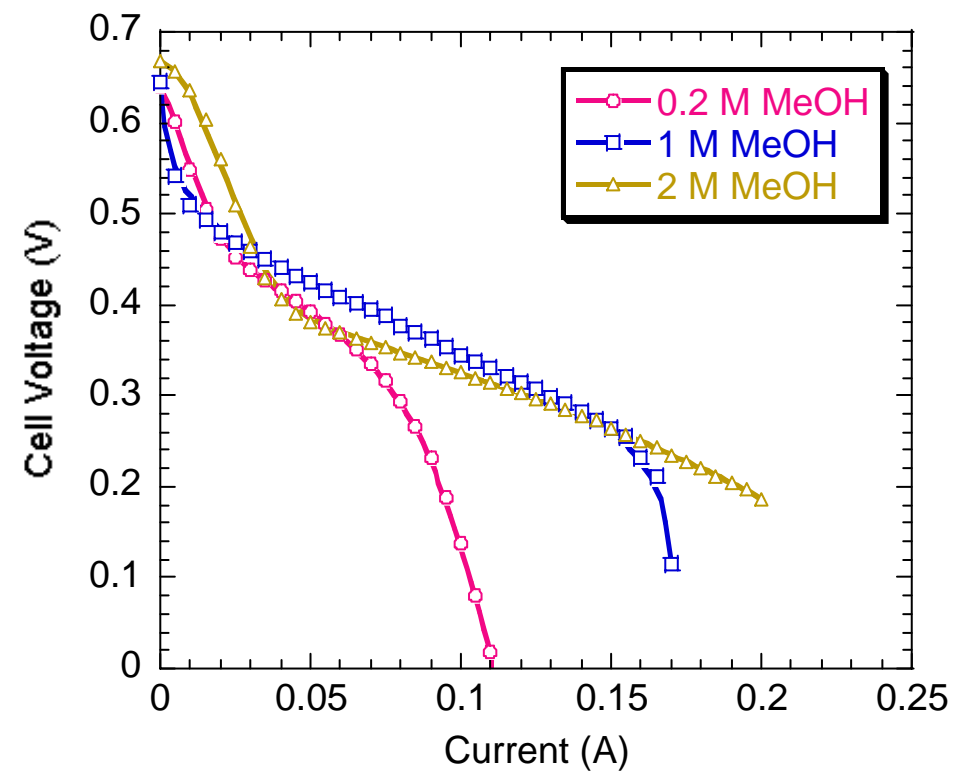

Figure 69 Measured characteristic curves of the DMFC device using different concentration of methanol water solution

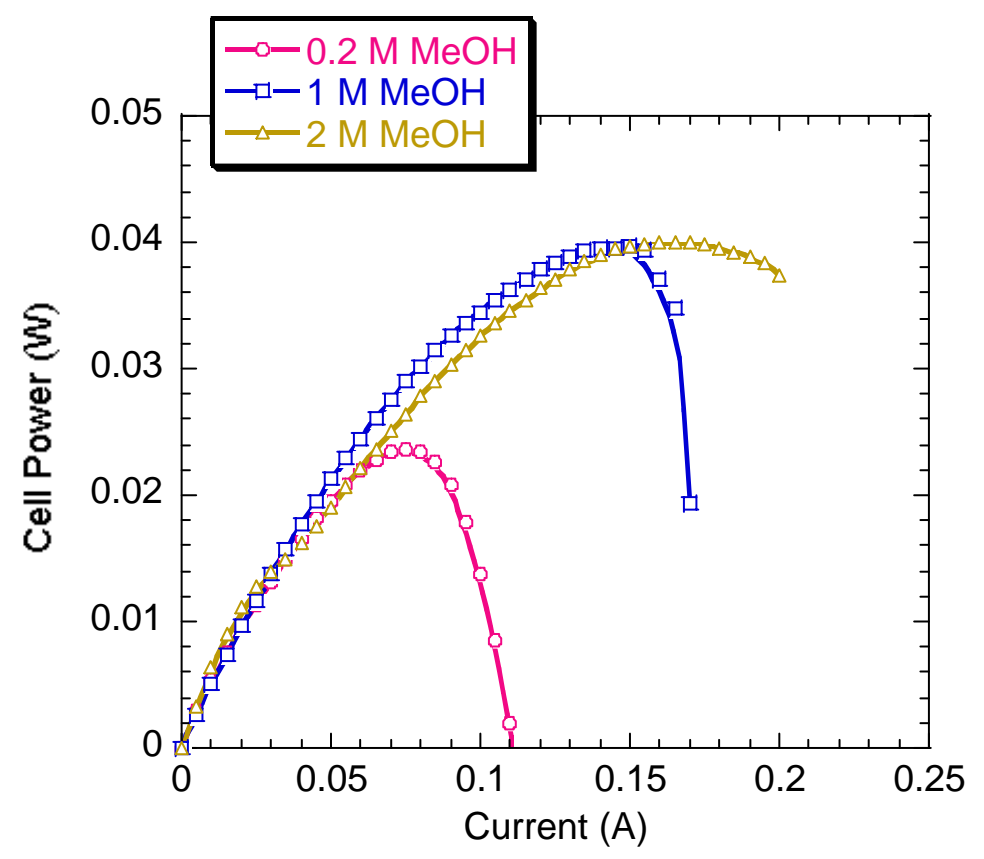

Figure 70 Calculated power of the DMFC device using different concentration of methanol water solution 
Fig. 69 shows the characteristic data of the DMFC device operating at room temperature with different fuel concentration. Also the fuel flow rate is equal to zero so the fuel is simply circulated by gravitational force. Since this DMFC device uses a meshed plate with complicated structure at the cathode side, it is very difficult to estimate the effective area for electrochemical reaction and the x-coordinate of the figure is current not current density. When the current is relatively larger, a steep performance drop can be observed for the cases using low and medium concentration of methanol water solution.

\subsubsection{Performance of the DMFC device driven by mini pump}

Fig. 71 and Fig. 72 show the comparison between the performance of DMFC running with a 5 $\mathrm{ml} / \mathrm{min}$ fuel flow rate and the performance with no forced fuel supply. Here the mini pump is used to supply the fuel flow rate. The concentration of the methanol water solution is $1 \mathrm{M}$ and the experiments are running at room temperature. A significant performance enhancement is observed for the case with $5 \mathrm{ml} / \mathrm{min}$ fuel flow rate. The maximum applicable current is increased and the maximum power is increased as well. This means that a forced fuel supply is necessary to improve the performance of the DMFC. The possible reasons for this performance enhancement includes avoidance of fuel depletion alone the anode surface, smaller resistance of the diffusion of methanol from bulk solution to the reaction interface, and better removal of carbon dioxide bubbles from the anode surface. From Fig. 72, it is found that the maximum power output of the fuel cell driven by mini pump is close to $50 \mathrm{~mW}$. It is roughly estimated that the power consumption of this pump is in the range of several watts to tens of watts. With the similar range of power output, the portable fuel cell power system can not sustain the operation of such pump by itself. Also the dimension of such pump is too large to make a compact system. 


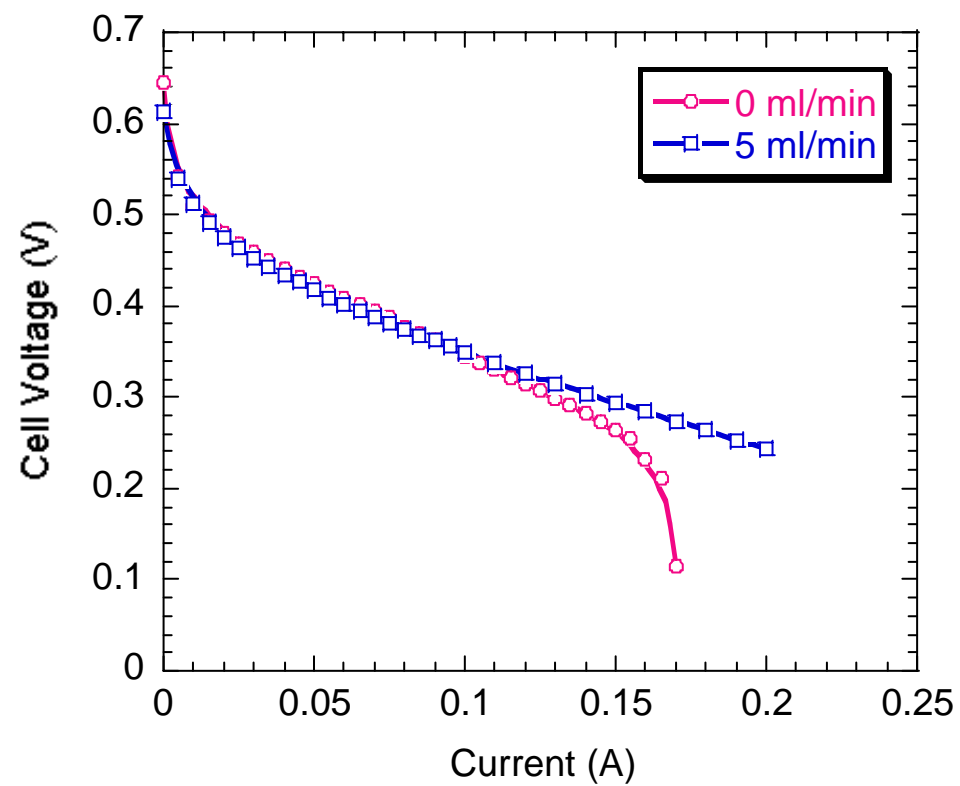

Figure 71 Measured characteristic curves of the DMFC device driven by mini pump

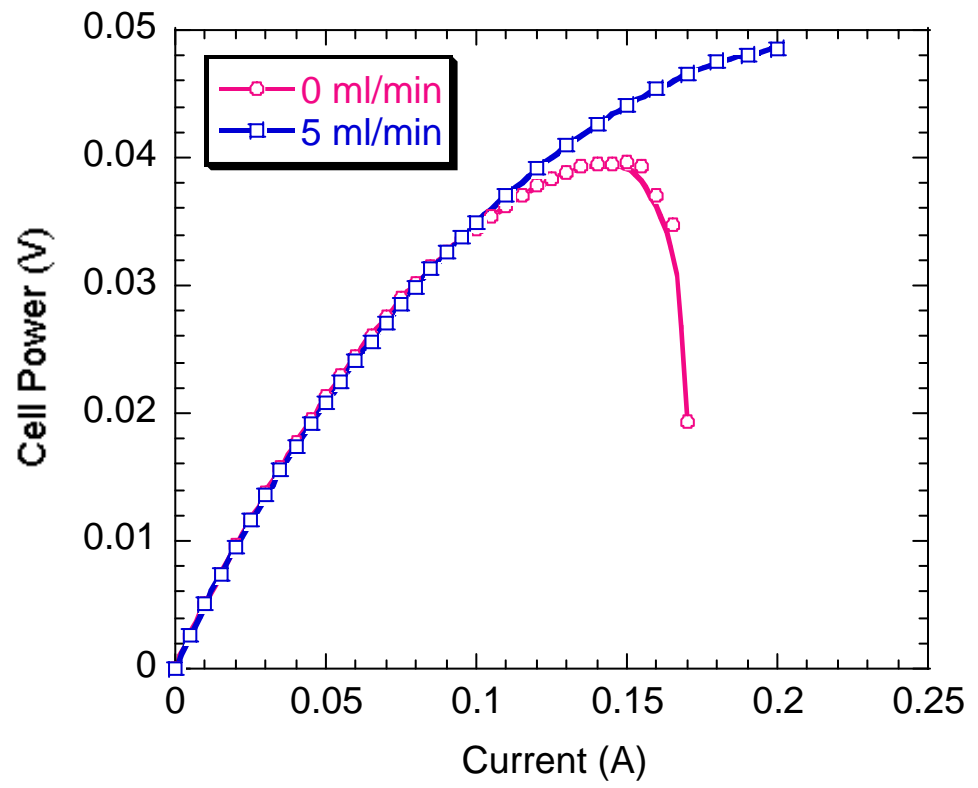

Figure 72 Calculated power of the DMFC device driven by mini pump 


\subsubsection{Performance of the DMFC device driven by micropump}

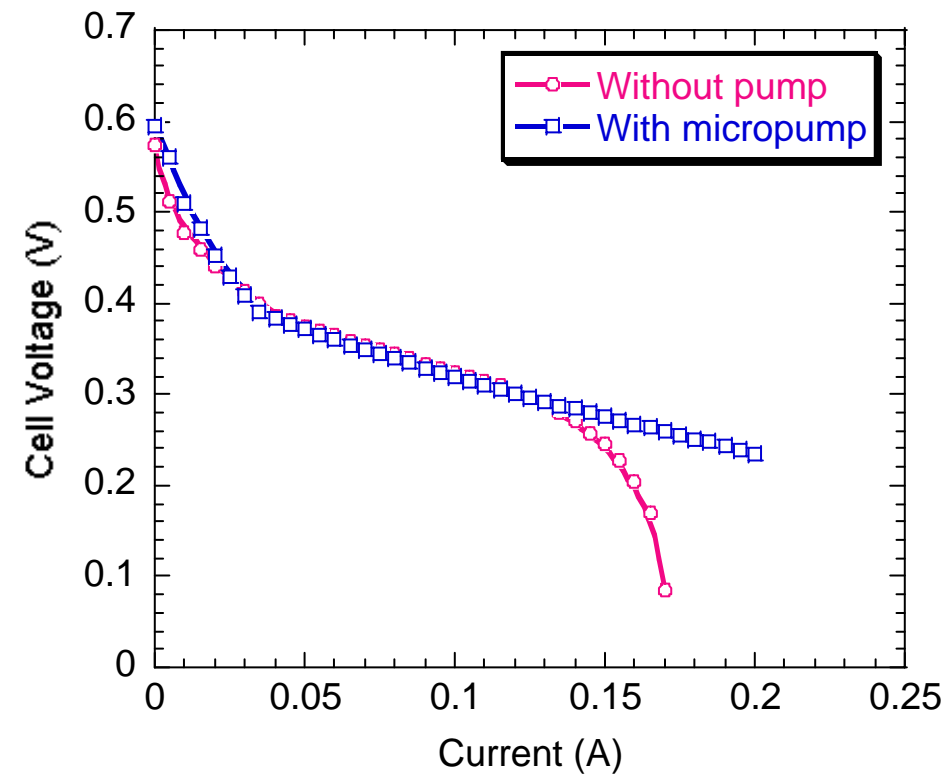

Figure 73 Measured characteristic curves of the DMFC device driven by micropump

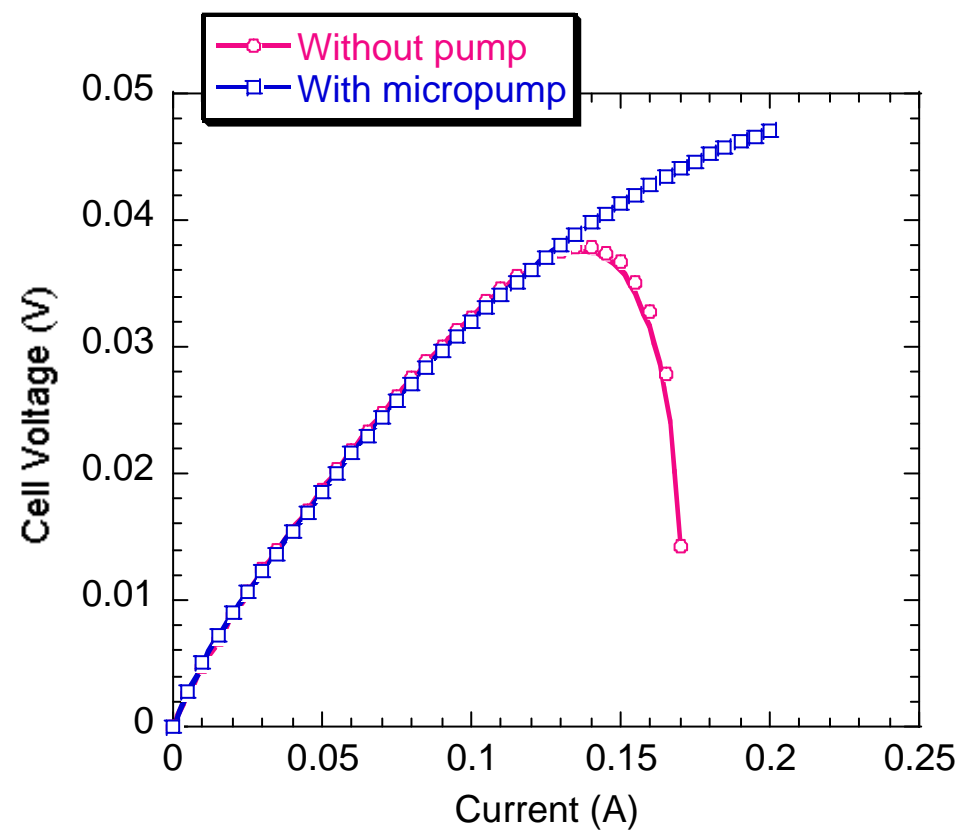

Figure 74 Calculated power of the DMFC device driven by micropump 
Fig. 73 and Fig. 74 show the comparison between the performance of DMFC driven by the micropump prototype and the performance with no forced fuel supply. The driving voltage is $100 \mathrm{~V}$ and the driving frequency is about $200 \mathrm{~Hz}$. The operation conditions are the same as the previous experiments but the fuel cell performance deteriorates a little bit due to the degradation of the materials. A significant performance enhancement can also be observed by using the micropump to supply fuel. Shown in Fig. 72, the maximum power output of the fuel cell driven by the micropump is also close to $50 \mathrm{~mW}$. And the power consumption of the micropump is in the range from $50 \mathrm{~mW}$ to $70 \mathrm{~mW}$ based on the theoretical calculation and experimental measurement presented in previous chapters. Although this means that the DMFC device used in this study still can not support the operation of the micropump prototype, it is possible to use the micropump in a DMFC portable power system with higher power output. Furthermore, as shown in the design introduced in Chapter 2, the micropump can be easily integrated with the fuel cell so the whole system can be very compact. Based on the previous theoretical analysis, the power consumption of the micropump can be further decreased to make it more applicable.

\subsection{DISCUSSION AND SUMMARY}

A lot of factors can affect the actual performance of a DMFC such as temperature, pressure, MEA materials, fuel cell structure and configuration, fuel supply, oxidant supply, water management, carbon dioxide removal and etc. Here some detailed discussions are given based on the fuel cell performance measurement presented in the previous chapters. 


\subsubsection{Open circuit voltage (OCV) of DMFC}

Based on Eq. (6.3), the ideal cell potential of the DMFC can be determined by the Nernst Equation:

$$
E=E^{0}+\frac{R T}{6 F} \ln \left(\frac{P_{\mathrm{O}_{2}}^{1.5}}{P_{\mathrm{CO}_{2}}}\right)
$$

Here, $E^{0}$ is the ideal standard potential for cell reaction. $R$ is the universal gas constant. $T$ is the Kelvin temperature. $F$ is the Faraday's constant. $P_{\mathrm{O}_{2}}$ represents the partial pressure of the oxygen and $P_{\mathrm{CO}_{2}}$ represents the partial pressure of the carbon dioxide. Using Eq. (6.5), the ideal cell potential of the DMFC is about $1.21 \mathrm{~V}$ under our experimental condition.

According to [73], in the absence of applied current, the open circuit voltage (OCV) is:

$$
O C V=E-\eta_{\text {xover }}
$$

Here, $\eta_{\text {xover }}$ is the potential loss due to the methanol crossover through the membrane.

The actual fuel cell voltage can be expressed as,

$$
V=E-\text { losses }=E-\eta_{\text {xover }}-\eta_{\text {act }}-\eta_{\text {ohm }}-\eta_{\text {diff }}=O C V-\eta_{\text {act }}-\eta_{\text {ohm }}-\eta_{\text {diff }}
$$

The OCV measured from our experiments varies between 0.6 and $0.7 \mathrm{~V}$ so the loss is pretty high. Note that not all of the loss comes from the methanol crossover as shown in Eq. (6.6). By comparing with the experimental data from [61], some of the loss is caused by the existence of a very small leakage current. Although this leakage current is very small, the loss is still very significant because the activation loss of the DMFC is high. More detail research is required to verify the loss due to methanol crossover and the loss due to the leakage current. 


\subsubsection{Influence of pumping}

Based on the experimental data, it is concluded that the performance of the DMFC can be improved by adding a pumping flow through the anode. The reasons for this may include avoidance of the fuel depletion, decreased diffusion resistance, and removal of the carbon dioxide.

During the operation of the DMFC, the methanol will be consumed by the electrochemical reaction happens along the anode side and the concentration will diminish. A forced fuel flow can circulated the fuel solution and provide fresh fuel to reaction site.

Also in fuel cell reaction, the concentration of the bulk solution and concentration of the solution at the anode interface are different. The methanol has to diffuse from the bulk solution to the anode/catalyst interface and the carbon dioxide has to migrate back to the bulk. In this process, diffusion resistance exists and the disturbance of the bulk solution due to the pumping can minimize this resistance.

The last factor, the removal of carbon dioxide is also very important to the performance of the DMFC. When the current is drawing out along the surface of the anode, the carbon dioxide will be generated at the same time. The size of the carbon dioxide bubbles will continue to grow if they are not removed from the anode surface. So when the reaction continues, more surfaces will be occupied by the carbon dioxide bubbles and the performance will deteriorate. A forced fuel flow can carry these carbon dioxide bubbles away when the size of them is still small. There exists a relationship between the flow rate and the size of the gas bubbles that can be removed with the flow. By calculating the buoyancy force of the gas bubble and the drag force by the fluid, some theoretical model can be developed to describe this problem. In our experiments, the 
anode side of the DMFC devices is covered with a transparent glass plate therefore the formation of the carbon dioxide bubbles can be visually observed. For the operations without pump, there are a lot of gas bubbles clinging to the anode surface when the current density is high or the operation time is long. With the help of pump, some of the bubbles can be removed and the accumulation of the bubbles at the anode surface is stable.

\subsubsection{Summary}

The performance of the DMFC driven by micropump has been investigated theoretically and experimentally. Results show that the idea is solid and the DMFC performance can be improved by using micropump. Possible reasons for this improvement are discussed. However, further research is still required to establish some quantitative relationship between the fuel cell performance and the micropump operation. Based on this relationship, it is possible to design and choose proper micropump for specific fuel cell power systems. In the next chapter, to improve the understanding of this complicated problem, electrochemical impedance spectroscopy (EIS) analysis method will be used. 


\subsection{ELECTROCHEMICAL IMPEDANCE SPECTROSCOPY (EIS) ANALYSIS}

\subsection{INTRODUCTION}

During the research and development of the miniaturized DMFC power system driven by piezoelectric valveless micropump, it is found that many factors can influence the actual performance of the fuel cell and impedance analysis can be a very useful tool to reveal the effects of these factors.

Impedance is a complex resistance encountered when a current flows through an electrical element. Impedance includes both magnitude and phase shift:

$$
Z=\frac{V}{I}=Z_{0}(\cos \phi+j \sin \phi)
$$

The electrochemical impedance spectroscopy (EIS) technique is based on the analysis of current response to small sinusoidal voltage perturbations, which is applied for a given frequency range from hundreds of $\mathrm{KHz}$ to the $\mathrm{mHz}$ scale. With EIS data, a complete description of an electrochemical system is possible. EIS is used in many areas of electrochemistry such as electrolytic process, corrosion, batteries and fuel cells. Data gathered by EIS can be used to extract information about transport properties, reaction kinetics, reaction mechanisms and ohmic losses.

Fig. 75 shows the example of an electrochemical reaction and its equivalent circuit. In (a), the oxidants with positive charges diffuse toward the electrode with negative charges. At the interface between the solution and the electrode the oxidants accept electrons and become 
neutral. Then the neutral reaction products diffuse back to the bulk solution. IHP and OHP are the inner and outer Helmholtz planes, respectively. The total impedance of this electrochemical reaction can be described by the equivalent circuit shown in (b).

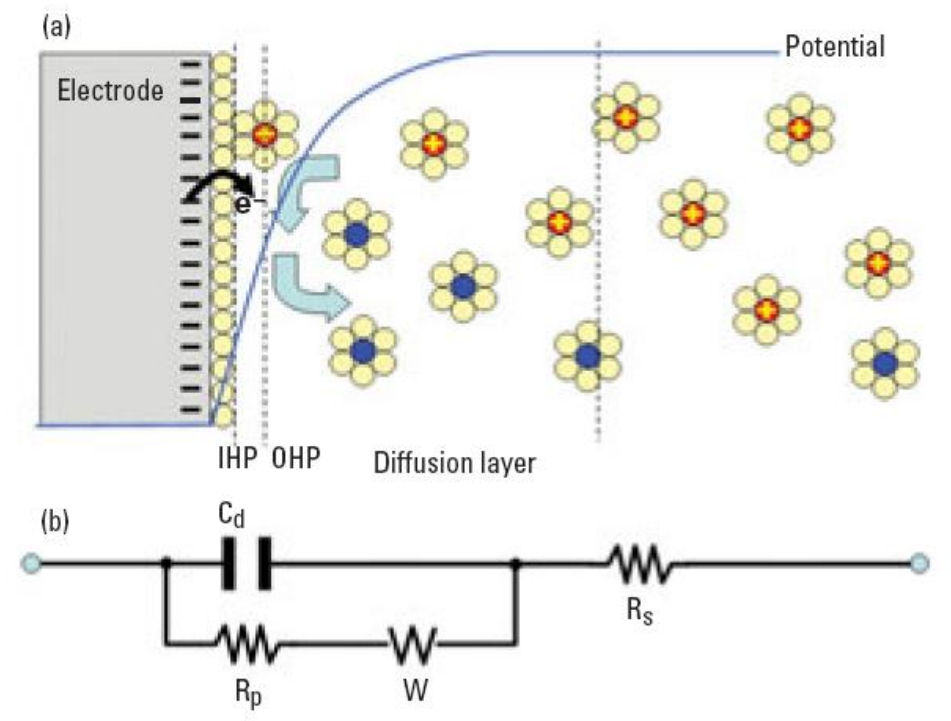

Figure 75 A simple electrochemical reaction and its equivalent circuit

$R_{s}$ represents the solution resistance, which depends on the ionic concentration, type of ions, temperature and the geometry of the area in which current is carried. $C_{d}$ represents the double layer capacitance. Because the charges in the electrode are separated from the charges of the ions in the solution, an electrical double layer forms at the interface between the electrode and its surrounding electrolyte. This double layer capacitance depends on the electrode potential, temperature, ionic concentrations, type of ions, and etc. $R_{p}$ is the polarization resistance. When the potential of an electrode is not equal to its value at open circuit, it can cause current to flow as a result of the electrochemical reactions occur at the electrode/electrolyte interface. The value of this current depends on the kind of the reaction, temperature, the concentration of the reaction products and the potential. The diffusion also creates impedance, which is called the Warburg impedance, $W$. This impedance depends on the frequency of the potential perturbation. At high 
frequencies the Warburg impedance is small since diffusion reactants don't have to move very far. At low frequencies the reactants have to diffuse farther and therefore the Warburg impedance is increasing. It is always difficult to calculate these impedance elements directly. Instead, they are often determined by fitting the equivalent circuit model with the experimental EIS data.

Fuel cell reactions are also electrochemical reactions and therefore EIS technique can be applied to the fuel cell research. An early review of the applications of EIS technique in MCFC, PAFC, SOFC and PEMFC has been presented in 1993 [62]. As reported by this review, EIS technique has been used to investigate the ionic conductivity (SOFC, PEMFC), in situ kinetics of electrode reactions (SOFC, MCFC) and electrode reaction mechanism (MCFC).

A common usage of EIS analysis in PEMFC is to characterize, evaluate and optimize the membrane electrode assembly (MEA) [63-65]. Recently a method has been developed to validate and evaluate the EIS data of PEMFC systems with state that change with time [66]. Based on this method, EIS technique was used to investigate the influence of carbon monoxide poisoning on the platinum anode [67]. A similar EIS study of the carbon monoxide tolerance for different Pt-alloy anode catalysts in PEMFC has been presented as well [68]. More comprehensive study of the impedance response of PEMFC has been conducted to investigate the effects of membrane thickness, cell temperature, and humidification conditions on the fuel cell performance [69]. And one of the latest EIS applications in fuel cell research is to study the water flooding in micro flow channels for fuel cells [70]. All of the researches introduced above are focused on PEMFC using hydrogen as fuel and there are only a few literatures [71] focused on the EIS study of DMFC using methanol water solution as fuel directly. It is expected that the EIS analysis of the DMFC system may reveal some useful information. 


\subsection{EXPERIMENTAL SETUP}

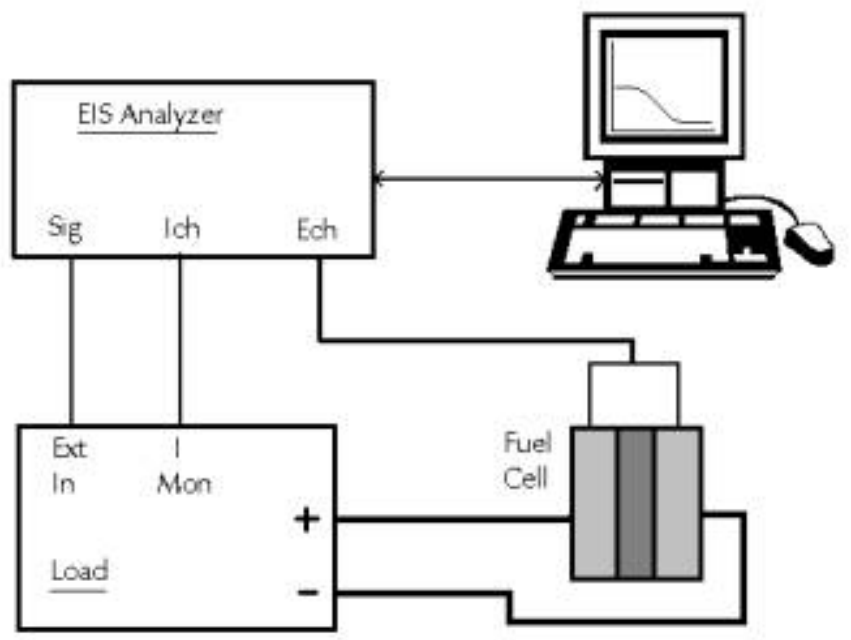

Figure 76 Experimental setup for the impedance measurement of a fuel cell device

Fig. 76 shows the experimental setup used to measure the EIS of a DMFC device. The DMFC device used here is the same as the one tested in the previous chapter and the photo of this device is shown in Fig. 67. A Camry EIS analyzer is used to measure the electrochemical impedance in the hybrid EIS mode, which can continually adjust the applied AC current so that the measured AC voltage does not extend beyond the linear range. In the experiments, the AC voltage is kept at $10 \mathrm{mV}$ (RMS value) and the frequency range is between $10 \mathrm{mHz}$ to $300 \mathrm{kHz}$, covered with 10 points per decade. A KEITHLEY 239 High Current Source Measure Unit is connected with the fuel cell to provide electronic load and a FLUKE 45 Dual Display Multimeter is used to monitor the cell voltage. A peristaltic Variable Flow Mini-pump is used to drive the methanol water solution into the fuel cell. The temperature can be adjusted by heating the methanol water solution using an isotemp digital stirring hotplate. 


\subsection{RESULTS AND ANALYSIS}

\subsubsection{Overall EIS of DMFC}

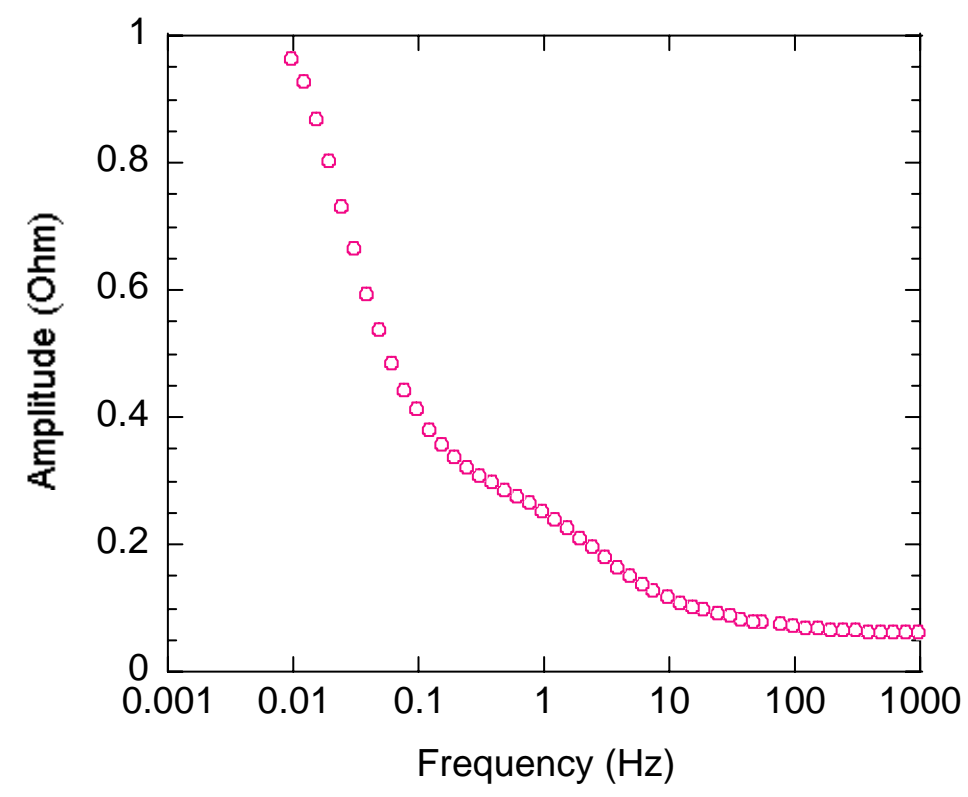

Figure 77 Measured impedance amplitude of a fuel cell device versus frequency

Shown in Fig. 77 and Fig 78 are the Bode plots of the measured impedance spectroscopy of the

DMFC device. The fuel cell is operating under room temperature and the output current is $0.1 \mathrm{~A}$.

The fuel flow rate is $0 \mathrm{ml} / \mathrm{min}$. In Fig. 77 the amplitude of the impedance are plotted against the frequency and in Fig. 78 the phase shift of the impedance are plotted against the frequency. It is found that with the increase of the frequency, the amplitude of the impedance converges to a stable value. Since the impedance of the double layer capacitance decreases when increasing the frequency, this value is believed to be the bulk resistance of the electrolyte membrane, as well as the contact resistance. The negative phase shift value in Fig. 78 also confirms the existence of the double layer capacitance. 


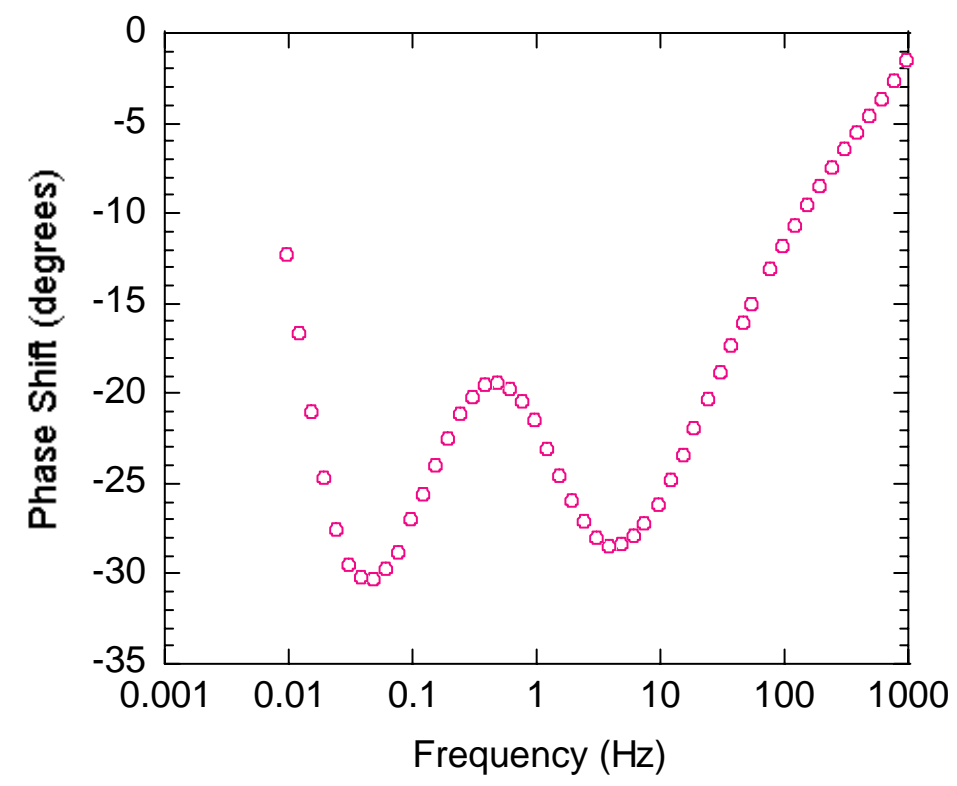

Figure 78 Measured impedance phase shift of a fuel cell device versus frequency

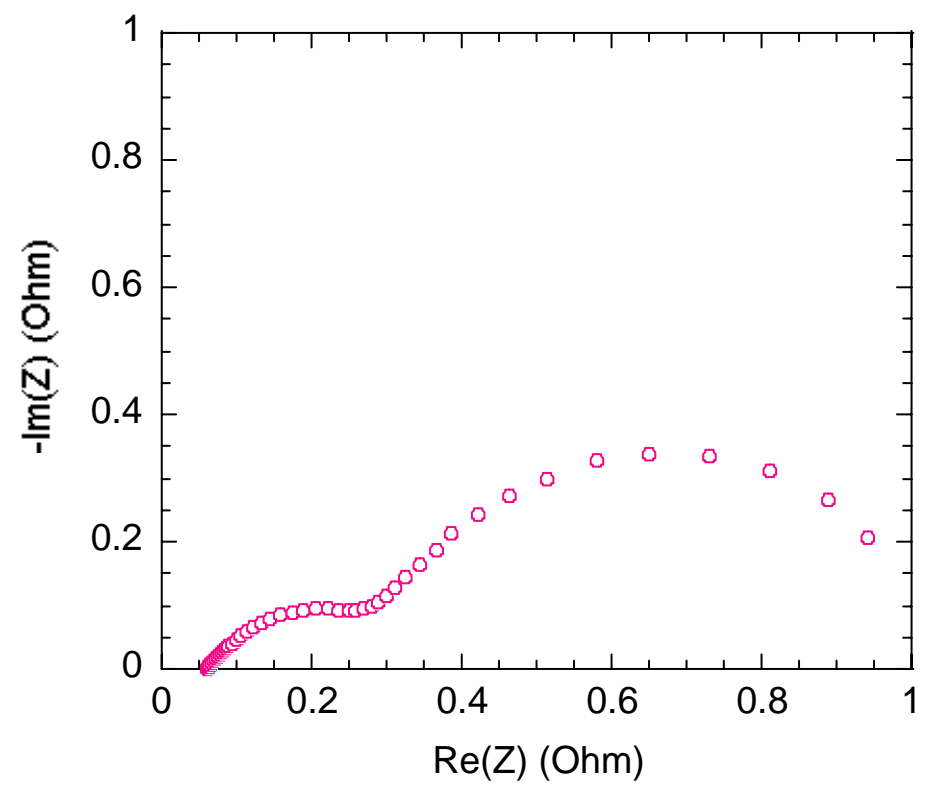

Figure 79 Nyquist plot of a fuel cell device

To identify and separate the different processes happening in the fuel cell reactions, it is useful to represent the measured impedance spectroscopy as a Nyquist diagram (imaginary part against real part of the impedance). As shown in Fig. 79, the measured Nyquist plot of the DMFC devices consists of two semi-circles approximately. The Medium-frequency (about $1 \mathrm{kHz} \sim 0.5$ 
$\mathrm{Hz}$ ) semi-circle, noted as R1, may correspond to the faster methanol electrooxidation kinetics and oxygen reduction reaction. The low-frequency semi-circle (below $1 \mathrm{~Hz}$ ), noted as R2, may be related to slower mass transfer of methanol at anode and mass transfer of oxygen at cathode. Both R1 and R2 are not perfect semicircles therefore there may be other factors contributing to the whole impedance spectrum such as interfacial effect and the roughness of the catalyst loaded electrodes. The interception of the impedance spectroscopy at the x-axis can be seen as the resistance of the electrolyte. As mentioned by some literature [71], a high-frequency semi-circle (higher than $1 \mathrm{kHz}$ ) related to ionic resistance of the membrane is missing which may be caused by device limitation or the pseudo induction effect from connection wires.

\subsubsection{Effect of output current}

Now the impedance spectroscopy is measured under different output current (operating at room temperature and $0 \mathrm{ml} / \mathrm{min}$ fuel flow rate). High output current corresponds to high overpotential so the effect of output current on EIS is also the effect of overpotential on EIS. Shown in Fig. 80 is the impedance spectroscopy measured at open circuit (no output current). It is interesting to see that the second semi-circle actually becomes a straight line. Then in Fig. 81, different impedance spectroscopy curves under different output currents (from 0.02 A to 0.18 A) are shown. It is obvious that the both semi-circles R1 and R2 shrink when increasing the output current. This is because the higher output current and the higher overpotential bring more electrochemical reactions. Also due to the consumption of the reactants, larger concentration gradients are formed between the interface and the bulk reactants. Therefore the diffusion processes are also accelerated. 
Note that the interceptions of all the impedance spectroscopy at the x-axis are almost the same since the bulk resistance of the electrolyte membrane doesn't change with respect to out put current. This value of the bulk resistance of the electrolyte membrane is about $0.06 \mathrm{Ohm}$.

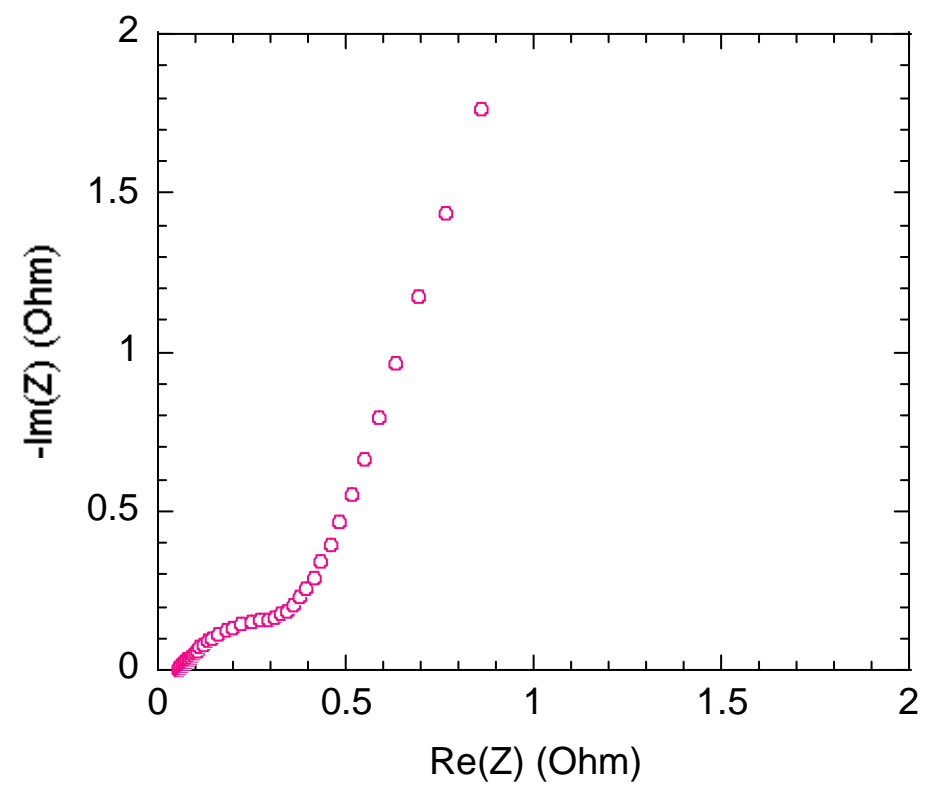

Figure 80 Measured Nyquist plot of a fuel cell device with open circuit (room temperature, no fuel flow rate)

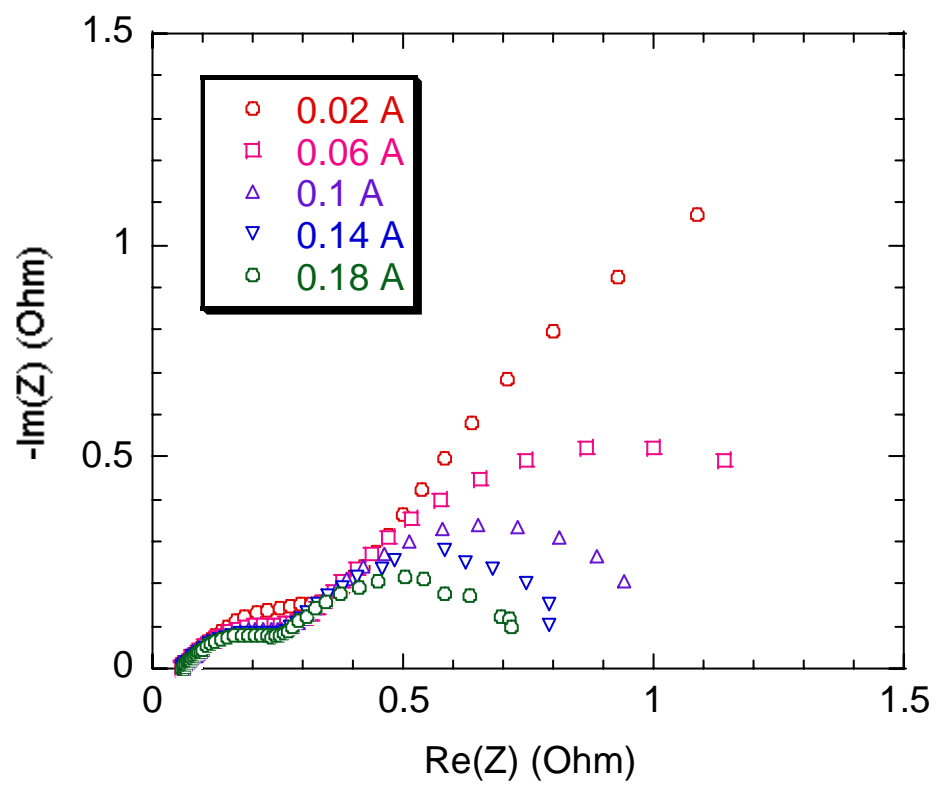

Figure 81 Measured Nyquist plot of a fuel cell device under different output current (room temperature, no fuel flow rate) 


\subsubsection{Effect of temperature}

Fig. 82 illustrates the variation of the impedance spectroscopy curves with different operation temperature. The output current is kept at $0.1 \mathrm{~A}$ and the fuel flow rate is $5 \mathrm{ml} / \mathrm{min}$. It is shown that increasing temperature can also result in the decease of R1 and R2. Also the effect of the temperature on R2 is more significant than the effect of the temperature on R1. The bulk resistance of the electrolyte doesn’t change much during this temperature range.

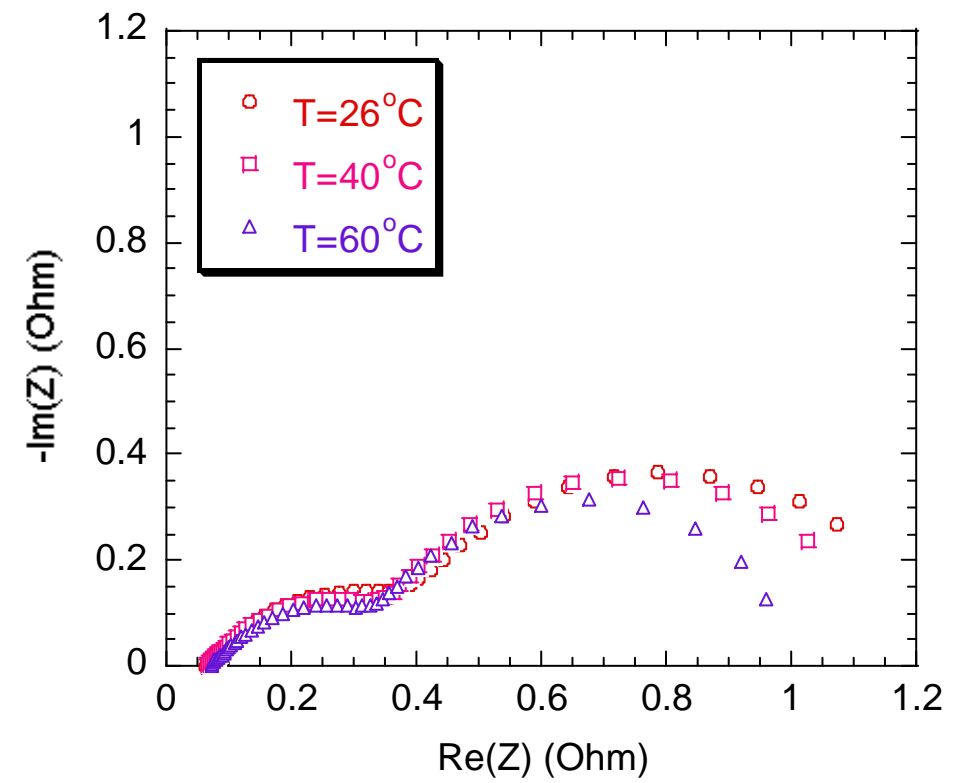

Figure 82 Measured Nyquist plot of a fuel cell device under different temperature (output current is 0.1 A, fuel flow rate is $5 \mathrm{ml} / \mathrm{min}$ )

\subsubsection{Effect of fuel flow rate}

Fig. 83 gives the variation of the impedance spectroscopy curves with different fuel flow rate. The output current is kept at $0.1 \mathrm{~A}$ and the fuel cell is working at room temperature. It is found that the effect of the fuel flow rate on the impedance spectroscopy is very small and negligible. 
However, for the impedance spectroscopy with $0 \mathrm{ml} / \mathrm{min}$ fuel flow rate, several irregular points with high impedance value appear at the low frequency end. The reason for this is that after a long time fuel cell operation, the reaction product carbon dioxide accumulates at the interface between the bulk fuel solution and the anode. These gad bubbles actually block the diffusion of the fresh fuel to the reaction site so the diffusion impedance is significantly increased. For the cases with non-zero fuel flow rate, these bubbles are partially removed with the fuel flow and the operation is more stable. Same as above, the bulk resistance of the electrolyte doesn't change under different fuel flow rate.

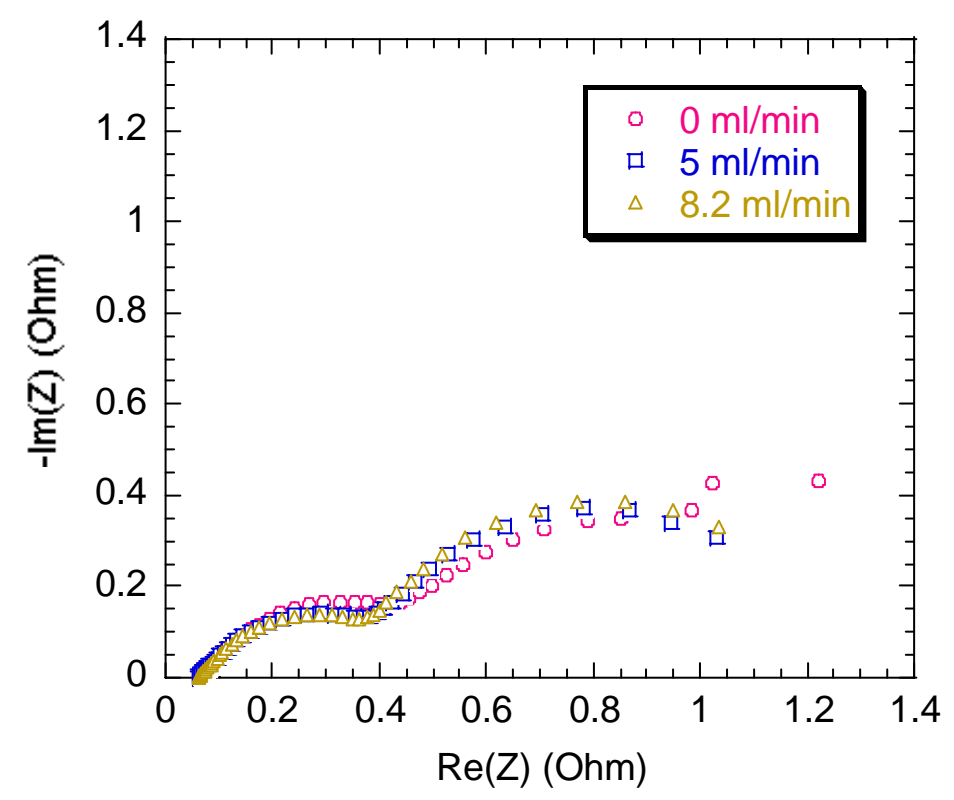

Figure 83 Measured Nyquist plot of a fuel cell device under different fuel flow rate (room temperature, output current is $0.1 \mathrm{~A}$ ) 


\subsubsection{Proposed equivalent circuit model}

To get a more quantitative analysis from the measured EIS data, it is necessary to establish an equivalent circuit (EC) model for the DMFC. An EC model for DMFC is proposed in Fig. 84. $R_{e}$ represents the resistance of the electrolyte membrane, which can be easily determined as

mentioned earlier. $C_{d, a}, R_{c t, a}$ and $W_{a}$ represent the double layer capacitance, the charge transfer resistance and the diffusion impedance at anode side, respectively. $C_{d, c}, R_{c t, c}$ and $W_{c}$ represent the double layer capacitance, the charge transfer resistance and the diffusion impedance at cathode side, respectively. Different components correspond to different processes that will happen in fuel cell reaction. There is some difficulty in identifying those impedance components using the measured EIS data. Since the current EIS data is the impedance of the whole fuel cell, there is no obvious way to separate the anode impedance and the cathode impedance from the whole impedance. Further research on this EIS analysis is still ongoing and hopefully this problem can be solved in the near future.

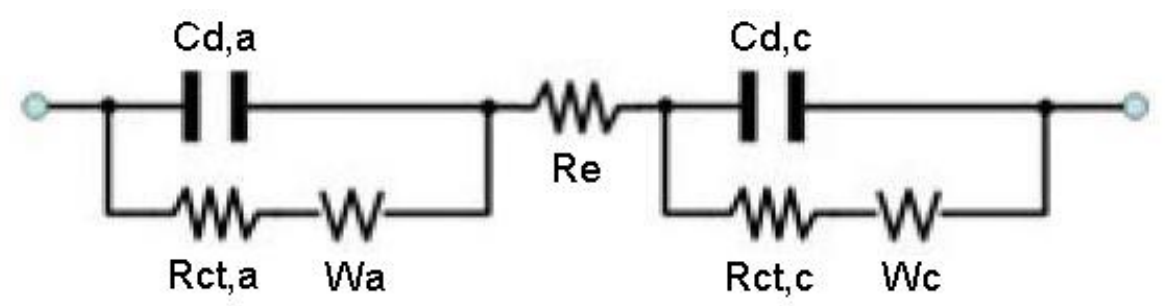

Figure 84 Proposed equivalent circuit model for DMFC 


\subsection{COCLUDING REMARKS}

This research is focused on the development of a valveless piezoelectric micropump for miniaturized direct methanol fuel cell system as power source for portable electronics. A theoretical and experimental study is conducted to design, optimize, and characterize such a system. This work demonstrates that the working principle of the whole system design is sound and viable. In addition, a powerful tool, electrochemical impedance spectroscopy analysis is employed to investigate the operation of DMFC.

\subsection{SUMMARY OF ACCOMPLISHMENTS}

The primary accomplishments of this research are listed below.

- A compact design of miniaturized DMFC power system driven by valveless piezoelectric micropump is presented.

- An analytical model to calculate the deflection of a PZT disk-type bending actuator caused by both electrical field and mechanical pressure is developed.

- An analytical model to calculate the deflection of a PZT ring-type bending actuator caused by both electrical field and mechanical pressure is developed. 
- An analytical model to establish the relationship between the applied voltage and the resultant flow rate, as well as power consumption for the micropump driven by PZT bending actuator is developed.

- Based on the theoretical analysis, the effects of the material properties and structure dimensions on the micropump performance are investigated.

- A valveless piezoelectric micropump prototype using PZT ring-type bending actuator is fabricated.

- The performance of the valveless piezoelectric micropump prototype without liquid loading is investigated experimentally.

- The performance of the valveless piezoelectric micropump prototype with liquid loading is investigated experimentally.

- The performance of a DMFC device operating with and without pump is investigated experimentally.

- The EIS data of a DMFC device under different output current, different temperature, and different fuel flow rate are measured.

- An equivalent circuit model that can be used to quantify the measured EIS data is proposed.

\subsection{FUTURE PERSPECTIVE}

The new design of the miniaturized DMFC driven by a valveless piezoelectric micropump is proved to be viable based on the current research results. To improve and optimize the performance of the real device, further modeling and experimental research are required. 
Specifically, a more accurate analytical model of the PZT ring-type bending actuator can be developed to determine the deflection induced by applied voltage. Also it is possible to develop a more complicated analytical model to take into account more accurately the coupling effects of liquid loading. From the experimental measurements, it is found that the maximum performance of this micropump is reached when the driving frequency is equal to the resonance frequency for both cases with and without liquid loading. Therefore, it is necessary to establish an improved dynamic model so that the actual performance of the micropump under the resonance frequency can be analyzed.

As for the experimental research, first a prototype combining the miniaturized DMFC with the valveless piezoelectric micropump has to be fabricated. More experimental data can be measured to evaluate the performance of the whole system when changing the operation conditions, including driving frequency, applied voltage, pressure head, various working fluid and etc.

To better understand the effects of pumping on the performance of the DMFC system, including the avoidance of fuel depletion, the decrease of the diffusion resistance, and the removal of carbon dioxide, some detailed quantitative research is required. It is planned that this work will be finished in the next several months and a paper will be submitted on this topic.

To improve the understanding of DMFC operation, it is also suggested that more detailed EIS analysis is required. One of the important issues that have to be solved is how to obtain the anode impedance and cathode impedance separately. It is believed that different processes involved in the fuel cell reaction can be quantified once the separated anode and cathode impedance data are available to be fit into the equivalent circuit model. 


\section{BIBLIOGRAPHY}

1. Hebling, C., Angelika Heinzel, and Ulf Groos, 2001, Technology and markets for portable fuel cells, Seventh Grove Fuel Cell Symposium, London, UK.

2. Waidhas, M., W. Drenckhahn, W. Preidel, H. Landes, 1996, Direct-fuelled fuel cells, Journal of Power Sources, Vol. 61, pp. 91-97.

3. Scott, K., W. M. Taama, P. Argyropoulos, 1999, Engineering aspects of the direct methanol fuel cell system, Journal of Power Sources, Vol. 79, pp. 43-59.

4. Heinzel, A., C. Hebling, M. Müller, M. Zedda, and C. Müller, 2002, Fuel cells for low power applications, Journal of Power Sources, Vol. 105, pp. 250-255.

5. Morse, Jeffrey D., Alan F. Jankowski, Robert T. Graff, and Jeffrey P. Hayes, 2000, Novel proton exchange membrane thin-film fuel cell for microscale energy conversion, J. Vac. Sci. Technol., Vol. 18(4), pp. 2003-2005.

6. Meyers, Jeremy P., and Helen L. Maynard, 2002, Design consideration for miniaturized PEM fuel cells, Journal of Power Sources, Vol. 109, pp. 76-88.

7. D’Arrigo, G., C. Spinella, G. Arena, and S. Lorenti, 2003, Fabrication of miniaturised Sibased electrocatalytic membranes, Materials Science and Engineering: C, Vol. 23, pp. 13-18.

8. Yu, Jingrong, Ping Cheng, Zhiqi Ma, Baolian Yi, 2003, Fabrication of miniature silicon wafer fuel cells with improved performance, Journal of Power Sources, Vol. 124, pp. 40-46.

9. Hahn, R., S. Wagner, A. Schmitz, H. Reichl, 2004, Development of a planar micro fuel cell with thin film and micro patterning technologies, Journal of Power Sources, Vol. 131, pp. 7378.

10. Mitrovski, Svetlana M., Lindsay C. C. Elliott, and Ralph G. Nuzzo, 2004, Microfluidic devices for energy conversion: planar integration and performance of a passive, fully immersed $\mathrm{H}_{2}-\mathrm{O}_{2}$ fuel cell, Langmuir, Vol. 20, pp. 6974-6976.

11. Holladay, Jamie, John Hu, Daniel Palo, Ya-Hui Chin, Ed Baker, Robert Dagle, 2002, Microfuel processors for a 15-25 watt fuel cell battery hybrid power supply, IECEC 2002, Paper No. 20074. 
12. Holladay, J. D., E. O. Jones, R. A. Dagle, G. G. Xia, C. Cao, Y. Wang, 2004, High efficiency and low carbon monoxide micro-scale methanol processors, Journal of Power Sources, Vol. 131, pp. 69-72.

13. Lee, S. J., A. Chang-Chien, S. W. Cha, R. O’Hayre, Y. I. Park, Y. Saito, and F. B. Prinz, 2002, Design and fabrication of a micro fuel cell array with "flip-flop" interconnection, Journal of Power Sources, Vol. 112, pp. 410-418.

14. Cha, S. W., R. O’Hayre, Y. Saito, F. B. Prinz, 2004, The scaling behavior of flow patterns: a model investigation, Journal of Power Sources, Vol. 134, pp. 57-71.

15. Gold, Scott, Kuan-Lun Chu, Chang Lu, Mark A. Shannon, Richard I. Masel, 2004, Acid loaded porous silicon as a proton exchange membrane for micro-fuel cells, Journal of Power Sources, Vol. 135, pp. 198-203.

16. Li, Jun, Christopher Moore, Paul A. Kohl, 2004, Investigation of acidic methanol solutions as a duel for microchannel fuel cells, Journal of Power Sources, Vol. 138, pp. 211-215.

17. Hsieh, C. C., S. C. Yao, Yousef Alyousef, 2003, Development of a silicon-based passive gasliquid separation system for microscale direct methanol fuel cells, Proceedings of IMECE’03, Paper No. 42135.

18. Karassik, Igor J., Joseph P. Messina, Paul Cooper, Charles C. Heald, 2001, Pump Handbook ( $3^{\text {rd }}$ Edition), McGraw-Hill, New York.

19. Gravesen, P., J. Brandebjerg, and O. Sondergard Jensen, 1993, Microfluidics - a review, Journal of Micromechanics and Microengineering, Vol. 4, pp. 168-182.

20. Smits, J. G., 1990, Piezoelectric micropump with three valves working peristaltically, Sensors and Actuators A, Vol. 21, pp. 304-306.

21. Laser, D. J., and J G Santiago, 2004, A review of micropumps, Journal of Micromechanics and Microengineering, Vol. 14, pp. R35-R64.

22. Van Lintel, H. T. G., F. C. M. van den Pol, and S. Bouwstra, 1988, A piezoelectric micropump based on micromachining in silicon, Sensors and Actuators, Vol. 15, pp. 153167.

23. Stemme, E., and G. Stemme, 1993, A valveless diffuser/nozzle-based fluid pump, Sensors and Actuators A, Vol. 39, pp. 159-167.

24. Gerlach, Torsten, M. Schuenemann, and Helmut Wurmus, 1995, A new micropump principle of the reciprocating type using pyramidic micro flow channels as passive valves, Journal of Micromechanics and Microengineering, Vol. 5, pp. 199-201.

25. Gerlach, Torsten, and Helmut Wurmus, 1995, Working principle and performance of the dynamic micropump, Sensors and Actuators A, Vol. 50, pp. 135-140. 
26. Forster, Fred K., Ronald L. Bardell, Martin A. Afromowitz, Nigel R. Sharma, Alan Blanchard, 1995, Design, fabrication and testing of fixed-valve micro-pumps, Proceedings of the ASME Fluids Engineering Division, FED-Vol. 234, pp. 39-44.

27. Olsson, Anders, G. Stemme, E. Stemme, 1995, A valve-less planar fluid pump with two pump chambers, Sensors and Actuators A, Vol. 46-47, pp. 549-556.

28. Olsson, Anders, P. Enoksson, G. Stemme and E. Stemme, 1996, A valve-less planar pump isotropically etched in silicon, Journal of Micromechanics and Microengineering, Vol. 6, pp. 87-91.

29. Olsson, Anders, P. Enoksson, G. Stemme and E. Stemme, 1997, Micromachined flat-walled valveless diffuser pumps, Journal of Microelectromechanical Systems, Vol. 6, No. 2, pp. 161-166.

30. Olsson, Anders, G. Stemme, E. Stemme, 1996, Diffuser-element design investigation for valve-less pumps, Sensors and Actuators A, Vol. 57, pp. 137-143.

31. Olsson, Anders, G. Stemme and E. Stemme, 1999, A numerical design study of the valveless diffuser pump using a lumped-mass model, Journal of Micromechanics and Microengineering, Vol. 9, pp. 34-44.

32. Olsson, Anders, G. Stemme and E. Stemme, 2000, Numerical and experimental studies of flat-walled diffuser elements for valve-less micropump, Sensors and Actuators A, Vol. 84, pp. 165-175.

33. Olsson, Anders, PhD Thesis: Valve-less Diffuser Micropumps, Royal Institute of Technology, Stockholm, Sweden, 1998.

34. Bardell, Ron L., Nigel R. Sharma, Fred K. Forster, Martin A. Afromowitz, Robert J. Penney, 1997, Designing high-performance micro-pumps based on no-moving-parts valves, Microelectromechanical Systems (MEMS) ASME, DSC-Vol. 62/HTD-Vol. 354, pp. 47-53.

35. Morris, Christopher J., and Fred K. Forster, 2000, Optimization of a circular piezoelectric bimorph for a micropump driver, Journal of Micromechanics and Microengineering, Vol. 10, pp. 459-465.

36. Koch, M., N. Harris, R. Maas, A. G. R. Evans, N. M. White and A. Brunnschweiler, 1997, A novel micropump design with thick-film piezoelectric actuation, Measurement Science and Technology, Vol. 8, pp. 49-57.

37. Koch, M., Nick Harris, Alan G. R. Evans, Neil M. White, Arthur Brunnschweiler, 1998, A novel micromachined pump based on thick-film piezoelectric actuation, Sensors and Actuators A, Vol. 70, pp. 98-103.

38. Koch, M., A. G. R. Evans and A. Brunnschweiler, 1998, The dynamic micropump driven with a screen printed PZT actuator, Journal of Micromechanics and Microengineering, Vol. 8, pp. 119-122. 
39. Nguyen, Nam-Trung, Xiaoyang Huang, 2001, Miniature valveless pumps based on printed circuit board technique, Sensors and Actuators A, Vol. 88, pp. 104-111.

40. Nguyen, Nam-Trung, Xiaoyang Huang, Numerical simulation of pulse-width-modulated micropumps with diffuser/nozzle elements, in: Proceedings of International Conference on Modeling and Simulation of Microsystems, San Diego, 2000, pp. 636-639.

41. Nguyen, Nam-Trung, Thai-Quang Truong, 2004, A fully polymeric micropump with piezoelectric actuator, Sensors and Actuators B, Vol. 97, pp. 137-143.

42. Ullmann, Amos, 1998, The piezoelectric valve-less pump-performance enhancement analysis, Sensors and Actuators A, Vol. 69, pp. 97-105.

43. Ullmann, Amos, and Ilan Fono, 2002, The piezoelectric valve-less pump-improved dynamic model, Journal of Microelectromechanical Systems, Vol. 11, No. 6, pp. 655-664.

44. Nedelcu, Oana Tatiana, Victor Moagar-Poladian, 1999, Modeling of the Piezoelectric Micropump for Improving the Working Parameters, Technical Proceedings 1999 International Conference on Modeling and Simulation of Microsystems.

45. Li, Shifeng, and Shaochen Chen, 2003, Analytical analysis of a circular PZT actuator for valveless micropumps, Sensors and Actuators A, Vol. 104, pp. 151-161.

46. Bu, Minqiang, Tracy Melvin, Graham Ensell, James S Wilkinson, Alan G. R. Evans, 2003, Design and theoretical evaluation of a novel microfluidic device to be used for PCR, Journal of Micromechanics and Microengineering, Vol. 13, pp. S125-S130.

47. Stemme, E., and Stig-Göran Larsson, 1973, The piezoelectric capillary injector-a new hydrodynamic method for dot pattern generation, IEEE Transaction on Electron Devices, Vol. ED-20, No. 1, pp. 14-19.

48. Dobrucki, A. B., P. Pruchnicki, 1997, Theory of piezoelectric axisymmetric bimorph, Sensors and Actuators A, Vol. 58, pp. 203-212.

49. Christensen, R. M., 1979, Mechanics of Composite Materials, John Wiley \& Sons, Inc., New York.

50. Timoshenko, S., S. Woinowsky-Krieger, 1959, Theory of Plates and Shells, second edition, McGraw-Hill Book Company, New York.

51. Koch, M., 1997, Silicon micromachined pumps employing piezoelectric membrane actuation for microfluidic system, PhD Dissertation, University of Southampton, pp. 69-76.

52. Kim, Sunghwan, William W. Clark, and Qing-Ming Wang, 2003, Piezoelectric energy harvesting using a diaphragm structure, Proceedings of SPIE, Vol. 5055, pp. 307-318.

53. Y. Kagawa and T. Yamabuchi, 1979, Finite Element Simulation of a Composite Piezoelectric Ultrasonic Transducer, IEEE Trans. Sonics and Ultrasonics, Vol. SU-2, No. 2, pp. 81-88. 
54. Mench, M. M., Z. H. Wang, K. Bhatia, and C. Y. Yang, 2001, Design of a micro direct methanol fuel cell ( $\mu$ DMFC), International Mechanical Engineering Congress and Exposition (IMECE), Nov. 11-16, 2001, New York, USA.

55. Shukla, A. K., P. A. Christensen, A. J. Dickinson, A. Hamnett, 1998, A liquid-feed solid polymer electrolyte direct methanol fuel cell operating at near-ambient conditions, Journal of Power Sources, Vol. 76, pp. 54-59.

56. Scott, K., P. Argyropoulos, P. Yiannopoulos, and W. M. Taama, 2001, Electrochemical and gas evolution characteristics of direct methanol fuel cells with stainless mesh flow beds, Journal of Applied Electrochemistry, Vol. 31, pp. 823-832.

57. Jung, D. H., Chang Hyeong Lee, Chang Soo Kim, Dong Ryul Shin, 1998, Performance of a direct methanol polymer electrolyte fuel cell, Journal of Power Sources, Vol. 71, pp. 169173.

58. Baldauf, M., W. Preidel, 1999, Status of the development of a direct methanol fuel cell, Journal of Power Sources, Vol. 84, pp. 161-166.

59. Dohle, H., J. Divisek, R. Jung, 2000, Process engineering of the direct methanol fuel cell, Journal of Power Sources, Vol. 86, pp. 469-477.

60. Amphlett, John C., Brant A. Peppley, Ela Halliop, Aamir Sadiq, 2001, The effect of anode flow characteristics and temperature on the performance of a direct methanol fuel cell, Journal of Power Sources, Vol. 96, pp. 204-213.

61. Gurau, Bogdan, Eugene S. Smotkin, 2002, Methanol crossover in direct methanol fuel cells: a link between power and energy density, Journal of Power Sources, Vol. 112, pp. 339-352.

62. Selman, J. R. and Y. P. Lin, 1993, Application of ac impedance in fuel cell research and development, Electrochimica Acta., Vol. 38, No. 14, pp. 2063-2073.

63. Wagner, N., 2002, Characterization of membrane electrode assemblies in polymer electrolyte fuel cells using a.c. impedance spectroscopy, Journal of Applied Electrochemistry, Vol. 32, pp. 859-863.

64. Romero-Castañón, T., L. G. Arriaga, U. Cano-Castillo, 2003, Impedance spectroscopy as a tool in the evaluation of MEA's, Journal of Power Sources, Vol. 118, pp. 179-182.

65. Song, J. M., S. Y. Cha, W. M. Lee, 2001, Optimal composition of polymer electrolyte furl cell electrodes determined by the AC impedance method, Journal of Power Sources, Vol. 94, pp. 78-84.

66. Schiller, C. A., F. Richter, E. Gülzow and N. Wagner, 2001a, Validation and evaluation of electrochemical impedance spectra of systems with states that change with time, Physical Chemistry Chemical Physics, Vol. 3, pp. 374-378. 
67. Schiller, C. A., F. Richter, E. Gülzow and N. Wagner, 2001b, Relaxation impedance as a model for the deactivation mechanism of fuel cells due to carbon monoxide poisoning, Physical Chemistry Chemical Physics, Vol. 3, pp. 2113-2116.

68. Leng, Yong-Jun, Xin Wang, I-Ming Hsing, 2002, Assessment of CO-tolreance for different Pt-alloy anode catalysts in a polymer electrolyte fuel cell using ac impedance spectroscopy, Journal of Electroanalytical Chemistry, Vol. 528, pp. 145-152.

69. Freire, Tatiana J. P., Ernesto R. Gonzalez, 2001, Effect of membrane characteristics and humidification conditions on the impedance response of polymer electrolyte fuel cells, Journal of Electroanalytical Chemistry, Vol. 502, pp. 57-68.

70. Cha, Suk Won, Ryan O’Hayre, and F. B. Prinz, AC impedance investigation of flooding in micro flow channels for fuel cells, Proceedings of Second International Conference on Fuel Cell Science, Engineering and Technology, Rochester, NY, June 14-16, 2004.

71. Mueller, Jens T., Peter M. Urban, 1998, Characterization of direct methanol fuel cells by ac impedance spectroscopy, Journal of Power Sources, Vol. 75, pp. 139-143.

72. Richter, M., R. Linnemann, P. Woias, 1998, Robust design of gas and liquid micropumps, Sensors and Actuators A, Vol. 68, pp. 480-486.

73. Barragán, V. M., A. Heinzel, 2002, Estimation of the membrane methanol diffusion coefficient from open circuit voltage measurements in a direct methanol fuel cell, Journal of Power Sources, Vol. 104, pp. 66-72. 